\title{
A molecular-line study of clumps with embedded high-mass protostar candidates
}

\author{
J. Brand ${ }^{1}$, R. Cesaroni ${ }^{2}$, F. Palla ${ }^{2}$, and S. Molinari ${ }^{3, \star}$ \\ 1 Istituto di Radioastronomia, CNR, Via Gobetti 101, 40129 Bologna, Italy \\ 2 Osservatorio Astrofisica di Arcetri, Largo E. Fermi 5, 50125 Florence, Italy \\ 3 Infrared Processing and Analysis Center, California Institute of Technology, MS 100-22, \\ Pasadena, CA 91125, USA
}

Received 2 October 2000 / Accepted 29 January 2001

\begin{abstract}
We present molecular line observations made with the IRAM 30-m telecopes of the immediate surroundings of a sample of 11 candidate high-mass protostars. These observations are part of an effort to clarify the evolutionary status of a set of objects which we consider to be precursors of UC HiI regions.

In a preceding series of papers we have studied a sample of objects, which on the basis of their IR colours are likely to be associated with compact molecular clouds. The original sample of 260 objects was divided approximately evenly into a High group, with IR colour indices [25-12] $\geq 0.57$ and [60-12] $\geq 1.3$, and a Low group with complementary colours. The FIR luminosity of the Low sources, their distribution in the IR colour-colour diagram, and their lower detection rate in $\mathrm{H}_{2} \mathrm{O}$ maser emission compared to the High sources, led to the hypothesis that the majority of these objects represent an earlier stage in the evolution than the members of the High group, which are mostly identifiable with UC HiI regions. Subsequent observations led to the selection of 12 Low sources that have FIR luminosities indicating the presence of B2.5 to O8.5 $V_{0}$ stars, are associated with dense gas and dust, have (sub-)mm continuum spectra indicating temperatures of $\sim 30 \mathrm{~K}$, and have no detectable radio continuum emission. One of these sources has been proposed by us to be a good candidate for the high-mass equivalent of a Class 0 object. In the present paper we present observations of the molecular environment of 11 of these 12 objects, with the aim to derive the physical parameters of the gas in which they are embedded, and to find further evidence in support of our hypothesis that these sources are the precursors to UC HiI regions. We find that the data are consistent with such an interpretation.

All observed sources are associated with well-defined molecular clumps. Masses, sizes, and other parameters depend on the tracer used, but typically the cores have average diameters of $\sim 0.5-1 \mathrm{pc}$ (with a range of 0.2 to $2.2 \mathrm{pc}$ ), and masses of a few tens to a few thousand solar masses. Compared to a similar analysis of High sources, the present sample has molecular clumps that are more massive, larger, cooler, and less turbulent. They also tend to have a smaller ratio of virial-to-luminous mass, indicating they are less dynamically stable than their counterparts in which the High sources are embedded. The large sizes suggest these clumps should still undergo substantial contraction (their densities are $\sim 10$ times smaller than those of the High sources). The lower temperatures and small linewidths are also expected in objects in an earlier evolutionary state. In various sources indications are found for outflowing gas, though its detection is hampered by the presence of multiple emission components in the line spectra. There are also signs of self-absorption, especially in the spectra of ${ }^{13} \mathrm{CO}$ and $\mathrm{HCO}^{+}$. We find that the masses of the molecular clumps associated with our objects increase with $\mathrm{L}_{\mathrm{fir}}\left(M_{\text {clump }} \propto L_{\mathrm{fir}}^{1.17}\right)$, and that there is a (weak) relation between the clump mass and the mass of the embedded protostellar object $M_{\text {proto }} \propto M_{\text {clump }}^{0.30}$. The large amount of observational data is necessarily presented in a compact, reduced form. Yet we supply enough information to allow further study. These data alone cannot prove or disprove the hypothesis that among these objects a high-mass protostar is truly present. More observations, at different wavelenghts and spatial resolutions are needed to provide enough constraints on the number of possible interpretations.
\end{abstract}

Key words. ISM: clouds - molecules, radio lines: ISM

Send offprint requests to: J. Brand,

e-mail: brand@ira.bo.cnr.it

* Present address: Istituto di Fisica dello Spazio Interplanetario, CNR, Via del Fosso del Cavaliere, 00133 Rome, Italy

\section{Introduction}

In spite of the importance of massive stars for the morphology and evolution of the Galaxy, and galaxies in general, the detailed study of the star formation process has 
mostly been concentrated on low-mass stars $\left(M<1 M_{\odot}\right)$. The reasons for this are evident: they are more abundant than high-mass stars, and the clouds in which they form are closer by, allowing a more detailed investigation. The study of high-mass $\left(M \geq 10 M_{\odot}\right)$ stars in their early evolutionary stages is furthermore complicated by the fact that they reach the ZAMS while still accreting material (and hence still suffering very high visual extinction), after which they rapidly destroy their natal environment.

In recent years however, ever growing efforts have been devoted to the study of the formation of high-mass stars, i.e. early type $(\mathrm{O}-\mathrm{B})$ stars with mass in excess of $\sim 10 \mathrm{M}_{\odot}$. In particular, attention has gradually shifted from the study of HII regions to that of ultracompact (UC) HII regions, to that of the molecular clumps in which UC HiI regions are embedded. Going from large, low-density structures to compact dense cores, corresponds to approaching the very earliest stages of the evolution of a massive star. Indeed, one of the goals of this type of research is to identify a massive stellar object in an evolutionary phase prior to the arrival on the main sequence, when most of its luminosity is derived from the release of gravitational energy. Such an object is defined a "protostar", and would observationally be recognized as an object of Class 0 (see André et al. 1994). These are most likely not to be found near UC HII regions, because an HII region rapidly alters (or even disrupts) the molecular cloud in which it forms. To find the initial conditions of star formation, one should therefore be looking for the precursors of UC HII regions.

\section{The search for massive protostars}

The observational approach followed by our group has been illustrated in a series of papers (Palla et al. 1991, 1993; Molinari et al. 1996, 1998a, 2000). A sample of 260 objects was selected from the IPSC, based on their FIR emission properties (for details see Palla et al. 1991). In particular, the IRAS colour criteria by Richards et al. (1987) were applied, which identify compact molecular clouds. This sample was then split into two sub-samples, based on their colour indices. The 125 sources with $[25-12] \geq 0.57$ and $[60-12] \geq 1.3$ comply with the Wood \& Churchwell (1989) criteria for identifying UC HII regions, and are called "High"; the remaining 135 sources are called "Low".

Based on their IR properties and the $\mathrm{H}_{2} \mathrm{O}$ maser occurrence frequency, we suggested that the Low sources are in an earlier evolutionary phase than the High sources (Palla et al. 1991). We have then performed a series of observations with a twofold goal: to confirm that the High sources are indeed UC HII regions, and - most importantly - to clarify the nature of the Low sources. This was accomplished by observations in many different tracers: $\mathrm{H}_{2} \mathrm{O}$ masers (Medicina telescope), $\mathrm{NH}_{3}(1,1)$ and $(2,2)$ lines (Effelsberg), continuum maps in the IR (ISO satellite), at centimeter (VLA), and (sub-) millimetre wavelengths (JCMT) (for a flowchart illustrating this process,

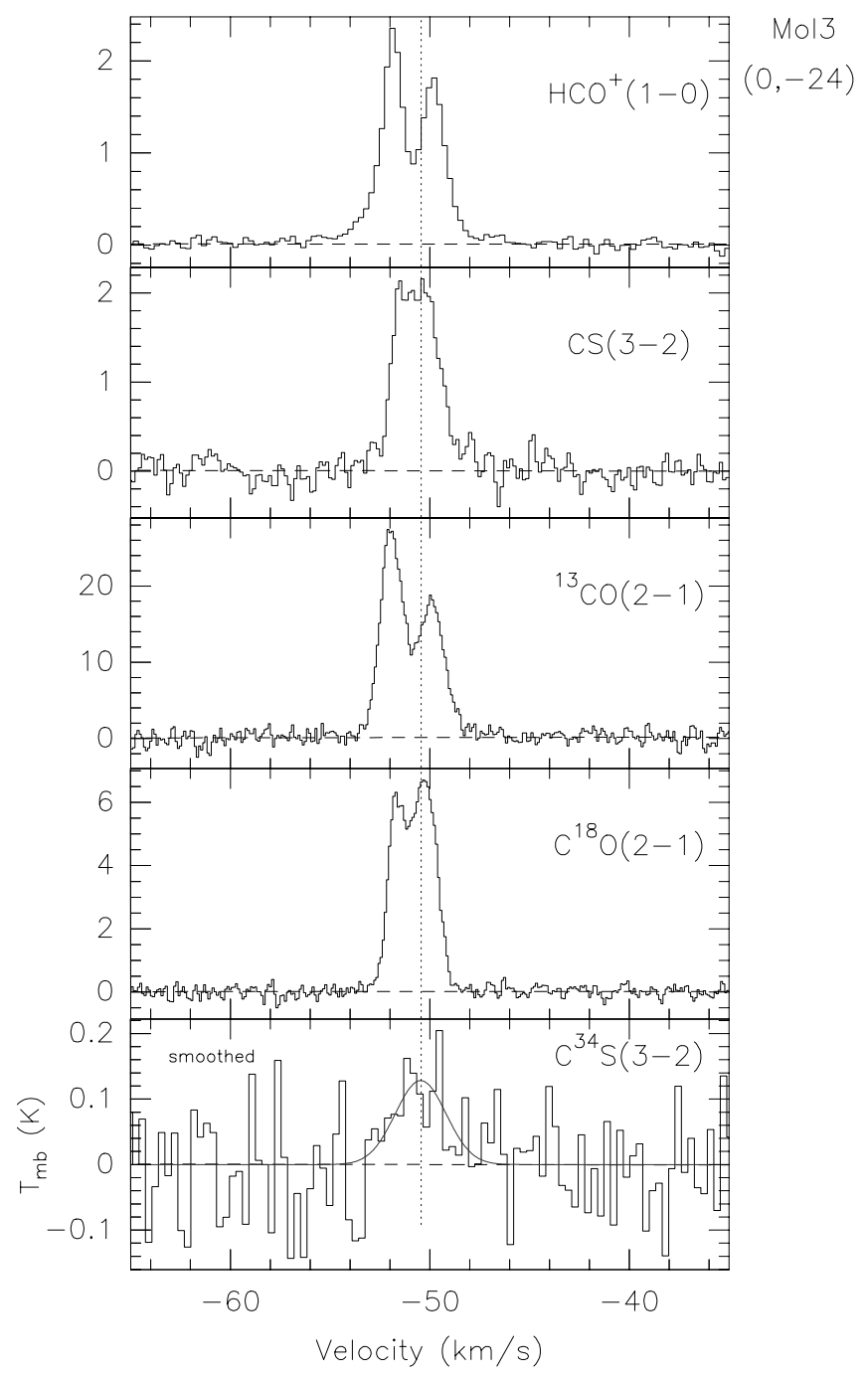

Fig. 1. a) Spectra towards the peak position in the maps for Mol 3. Source name, offset position, and molecules/transitions are indicated. The offset is relative to the maps' central position, which is the sub-mm peak (Molinari et al. 2000). The main beam brightness temperature $\left(T_{\mathrm{mb}}\right)$ is plotted against $V_{\text {lsr }}$. The vertical dotted line indicates the $V_{\text {lsr }}$ of the $\mathrm{C}^{34} \mathrm{~S}(3-2)$ line, obtained from a Gaussian fit; the horizontal dashed lines indicate the $T_{\mathrm{mb}}=0 \mathrm{~K}$ level in each spectrum

see Molinari et al. 2000, Fig. 1). The main results of these observations can be summarized as follows:

- High and Low sources have luminosities typical of highmass stars, with the latter being only slightly less luminous than the former;

- $\mathrm{H}_{2} \mathrm{O}$ masers are much more common towards High than Low sources (detection rates $26 \%$ and $9 \%$, respectively);

- $\mathrm{NH}_{3}$ emission is detected towards both samples (with a detection rate of $45 \%$ for Low and $80 \%$ for High sources), although the temperatures derived from the ratio of the $(1,1)$ and $(2,2)$ lines indicate that Low sources are slightly colder than High; 


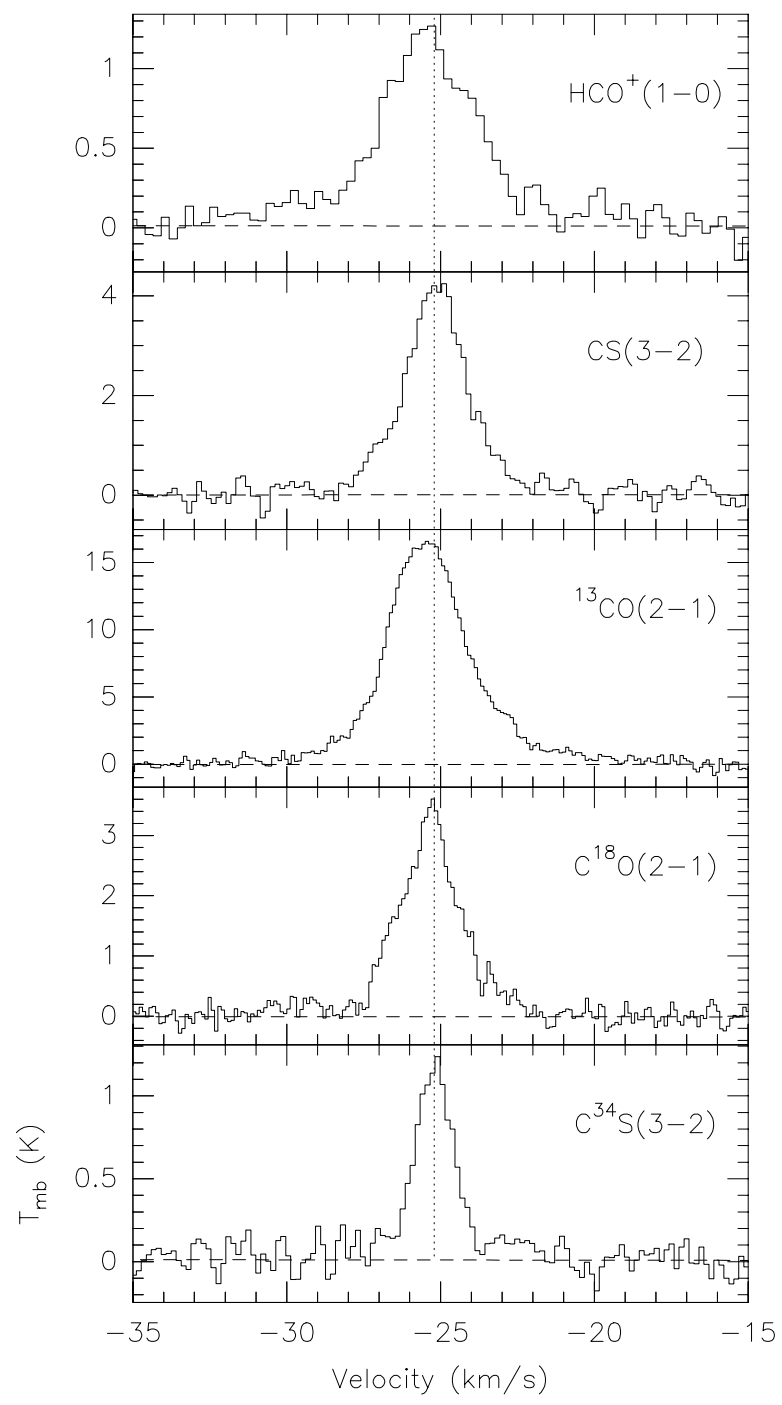

Fig. 1. b) As a), for Mol 8

- Free-free emission is detected towards $43 \%$ of the High, but towards only $24 \%$ of the Low sources observed with high angular resolution with the VLA (at 2 and $6 \mathrm{~cm}$ );

- For 17 out of 30 (57\%) of the Low sources mapped with the JCMT, the (sub-) millimeter continuum emission arises from a compact $\left(\sim 30^{\prime \prime}\right)$ region around the IRAS source; total core masses are typically 10-200 $M_{\odot}$;

- The continuum spectrum of the Low sources between 0.35 and $2 \mathrm{~mm}$ indicates temperatures of $\sim 30 \mathrm{~K}$, significantly lower than those, measured towards "hot cores": small $(\sim 0.1 \mathrm{pc})$, dense $\left(\sim 10^{7} \mathrm{~cm}^{-3}\right)$, hot $(\gtrsim 100 \mathrm{~K})$, and luminous $\left(L \gtrsim 10^{4} L_{\odot}\right)$ molecular condensations, which very likely host high-mass objects that are already on the main sequence, but that are still too young to have developed an UC HiI region.

The main conclusions one can draw are that High sources are UC HiI regions, and that Low sources are associated with massive stars (as suggested by their luminosities), but do not show any of the tracers typical of the

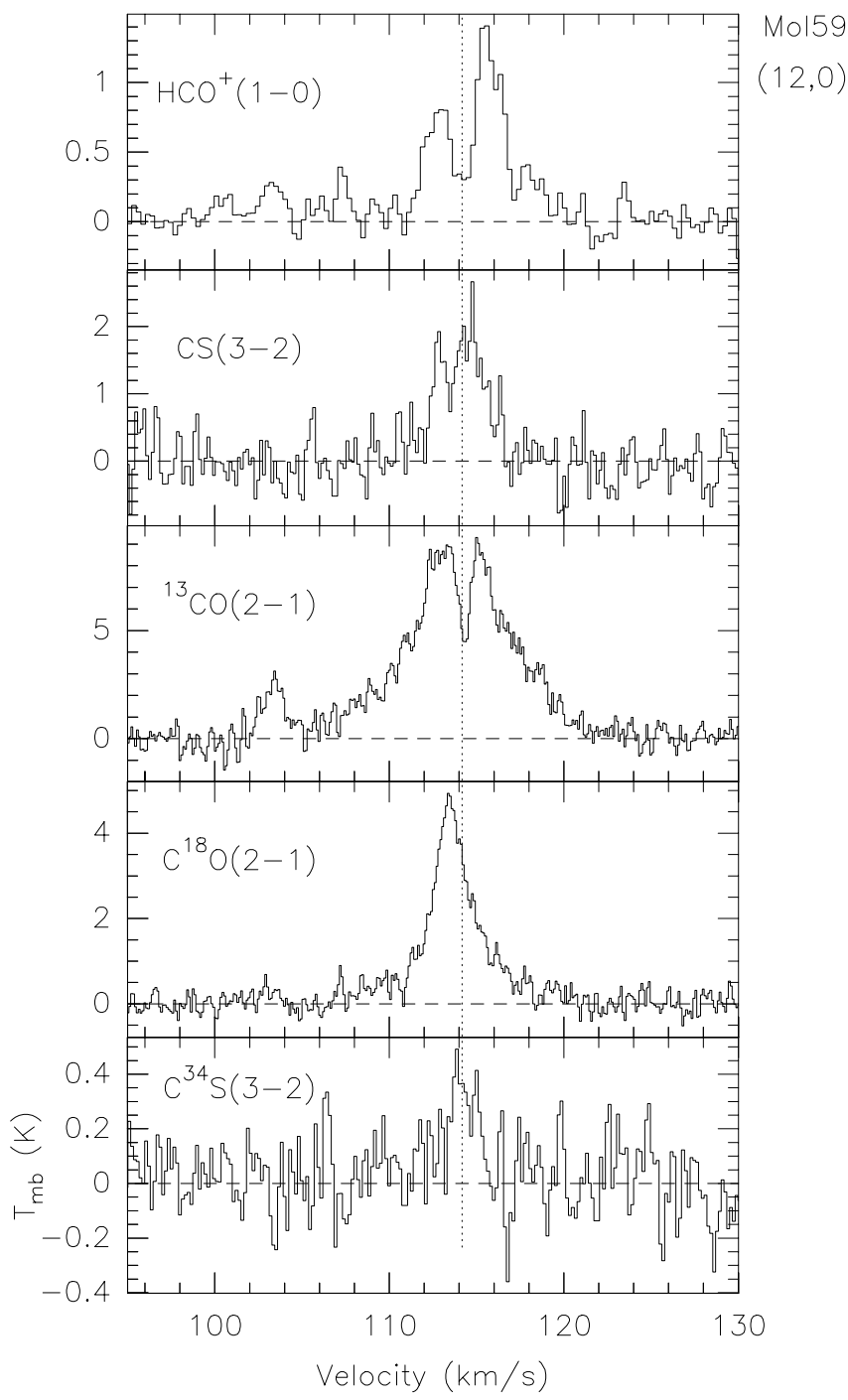

Fig. 1. c) As a), for Mol 59

molecular cores hiding a main sequence early type star (such as compact free-free emission, high kinetic temperature).

With the aim of establishing an evolutionary sequence for our sources, we have started a mapping survey with the NRAO 12-m at Kitt Peak. Two phenomena often associated with protostars (of all masses) are $\mathrm{H}_{2} \mathrm{O}$ masers and molecular outflows. The association of $\mathrm{CO}$ outflows with UC Hir regions has been well established by Shepherd \& Churchwell (1996). However, surveys show that $\mathrm{H}_{2} \mathrm{O}$ masers can appear slightly before the development of a detectable HII region (Codella et al. 1996, 1997), and that they are also closely associated with CO outflows (Felli et al. 1992; see also Wouterloot et al. 1995, for a statistical analysis of the correlations between FIR, $\mathrm{H}_{2} \mathrm{O}$, and $\mathrm{CO}$ emission). From maps of the outflows, one can determine the dynamical timescale, which provides a lower limit to the protostellar age. Combining the presence or absence of 


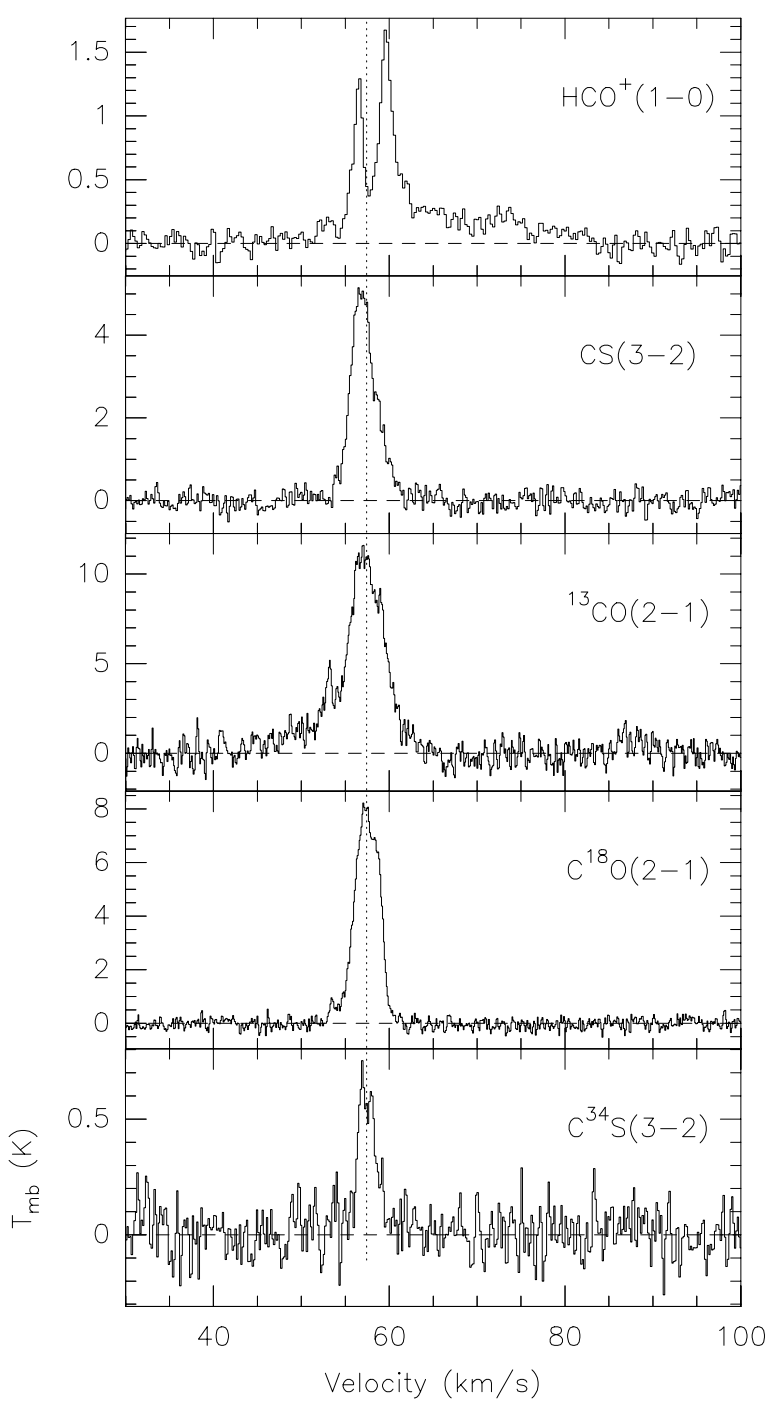

Fig. 1. d) As a), for Mol 75

$\mathrm{H}_{2} \mathrm{O}$ maser- and radio continuum emission should provide a rough measure of the age of the source.

Interferometric (mm-continuum, and -lines) are needed to study the envelopes and (possible) disks of the embedded objects. Single-dish observations are important to study the physical and kinematical properties of the molecular cores, in which the candidate protostellar objects are embedded. A study of the kinematic properties is especially important: protostars derive the major part of their luminosity from accretion, and one therefore expects to see signatures of infall towards these sources (see e.g. Myers et al. 2000, and references therein). Although high-resolution interferometric observations are needed for a complete investigation, as a first step the resolution provided by a telescope like the IRAM 30-m will suffice to study the physical state of the sources, and to derive a rough estimate for the size and mass of the associated molecular material. Such observations are reported in the present paper.

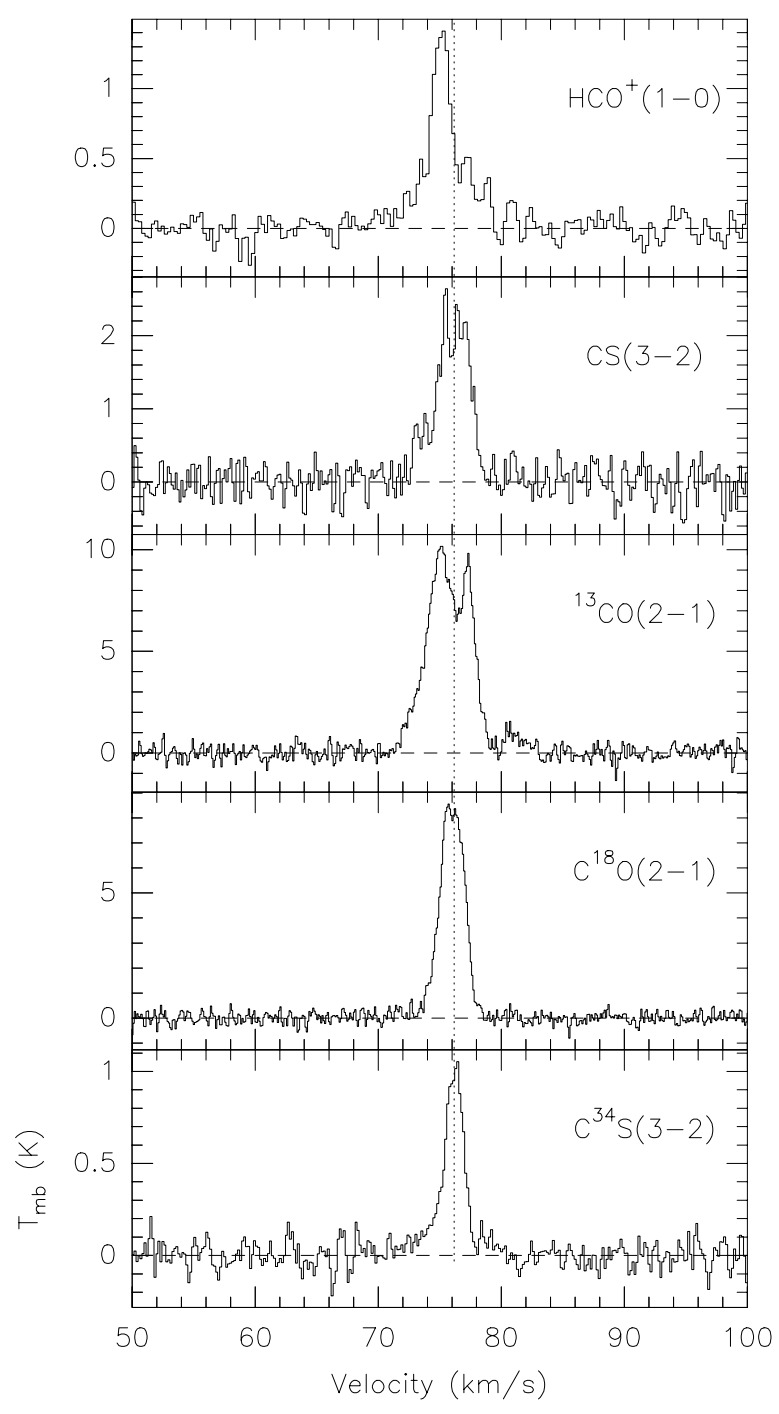

Mol77

Fig. 1. e) As a), for Mol 77

Table 1. Observed transitions

\begin{tabular}{|c|c|c|c|c|}
\hline Molecule & $\begin{array}{c}\text { Frequency } \\
(\mathrm{MHz})\end{array}$ & $\begin{array}{c}V_{\mathrm{res}}^{1} \\
\left(\mathrm{~km} \mathrm{~s}^{-1}\right)\end{array}$ & $\begin{array}{c}H P B W \\
\left({ }^{\prime \prime}\right)\end{array}$ & Notes \\
\hline \multicolumn{5}{|c|}{ IRAM $30-\mathrm{m}$} \\
\hline $\mathrm{HCO}^{+}(1-0)$ & 89188.518 & 0.26 & 27 & $\mathrm{a}$ \\
\hline${ }^{13} \mathrm{CH}_{3} \mathrm{CN}(5-4)$ & 89331.297 & 3.36 & 27 & $a, b$ \\
\hline $\mathrm{CH}_{3} \mathrm{C}_{2} \mathrm{H}(6-5)$ & 102547.984 & 0.22 & 23 & $\mathrm{~b}$ \\
\hline $\mathrm{C}^{34} \mathrm{~S}(3-2)$ & 144617.147 & 0.16 & 17 & $\mathrm{c}$ \\
\hline $\mathrm{CH}_{3} \mathrm{OH}\left(\mathrm{V}_{t}=1\right)$ & 145103.230 & 2.07 & 17 & c \\
\hline $\mathrm{CS}(3-2)$ & 146969.049 & 0.16 & 16 & $\mathrm{~d}$ \\
\hline $\mathrm{CH}_{3} \mathrm{CN}(8-7)$ & 147174.592 & 2.04 & 16 & $\mathrm{~b}, \mathrm{~d}$ \\
\hline $\mathrm{C}^{18} \mathrm{O}(2-1)$ & 219560.328 & 0.11 & 11 & \\
\hline${ }^{13} \mathrm{CO}(2-1)$ & 220398.686 & 0.11 & 11 & e \\
\hline $\mathrm{CH}_{3} \mathrm{CN}(12-11)$ & 220747.268 & 1.36 & 11 & $\mathrm{~b}, \mathrm{e}$ \\
\hline \multicolumn{5}{|c|}{ KOSMA 3-m } \\
\hline $\mathrm{HCO}^{+}(4-3)$ & 356734.253 & 0.14 & 74 & \\
\hline
\end{tabular}

\footnotetext{
${ }^{1}$ Highest velocity resolution available; a,c,d,e Transitions measured in same receiver; b Frequency of the $K=0$ transition.
} 


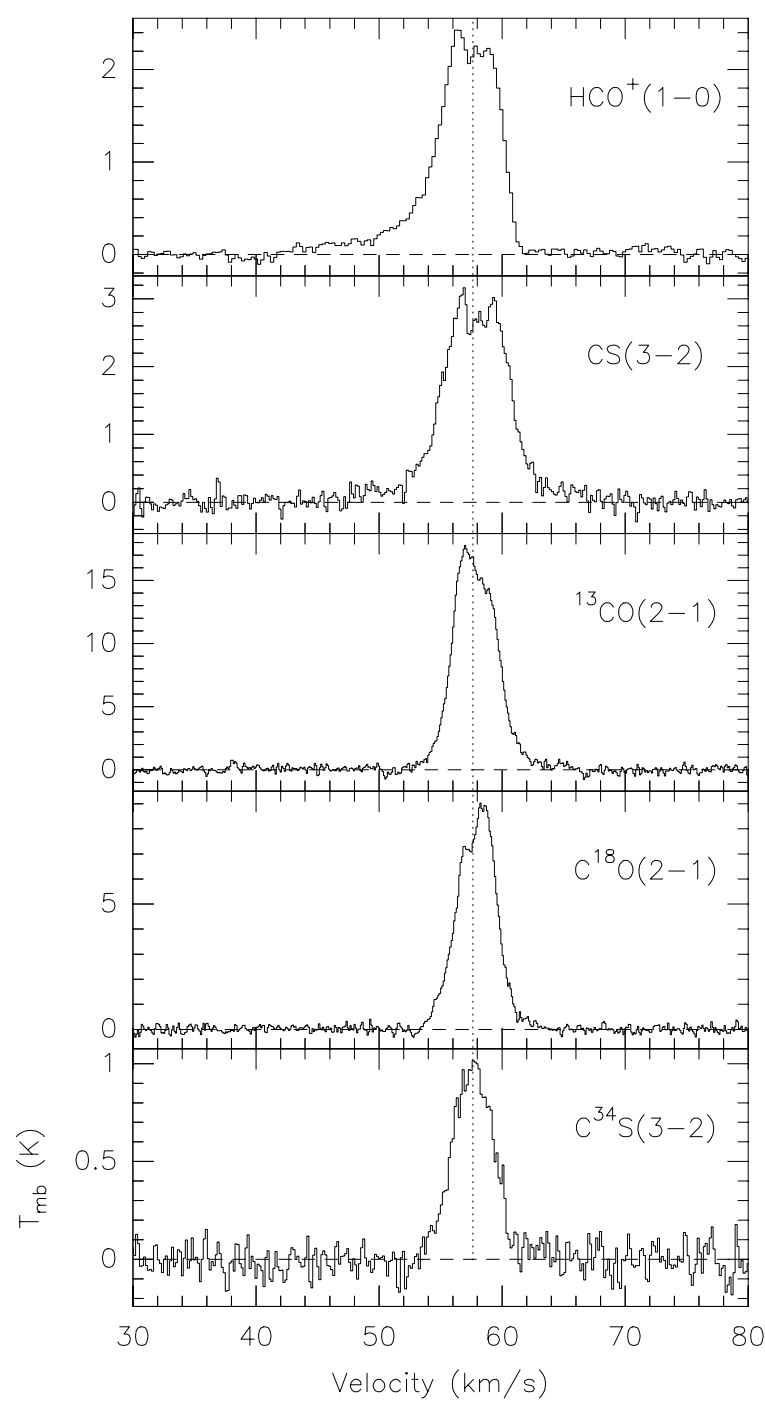

Fig. 1. f) As a), for Mol 98

In Sect. 3 the observations are described; the data are presented in Sect. 4. Individual sources are commented in Sect. 5, and the results are summarized and compared with those of High sources in Sect. 6 .

\section{Observations}

\subsection{IRAM}

Observations were carried out between October 10-12, 1997, with the IRAM 30-m telescope at Pico Veleta (Granada, Spain). We used three SIS receivers simultaneously, in combination with the $100 \mathrm{kHz}$ and $1 \mathrm{MHz}$ resolution filterbanks, and the autocorrelator, split into as many as five parts. The observed molecules and frequencies are listed in Table 1, where we also indicate the molecules that were observed simultaneously with the same receiver; this was achieved by tuning the receiver to a frequency intermediate between those of the two transitions.

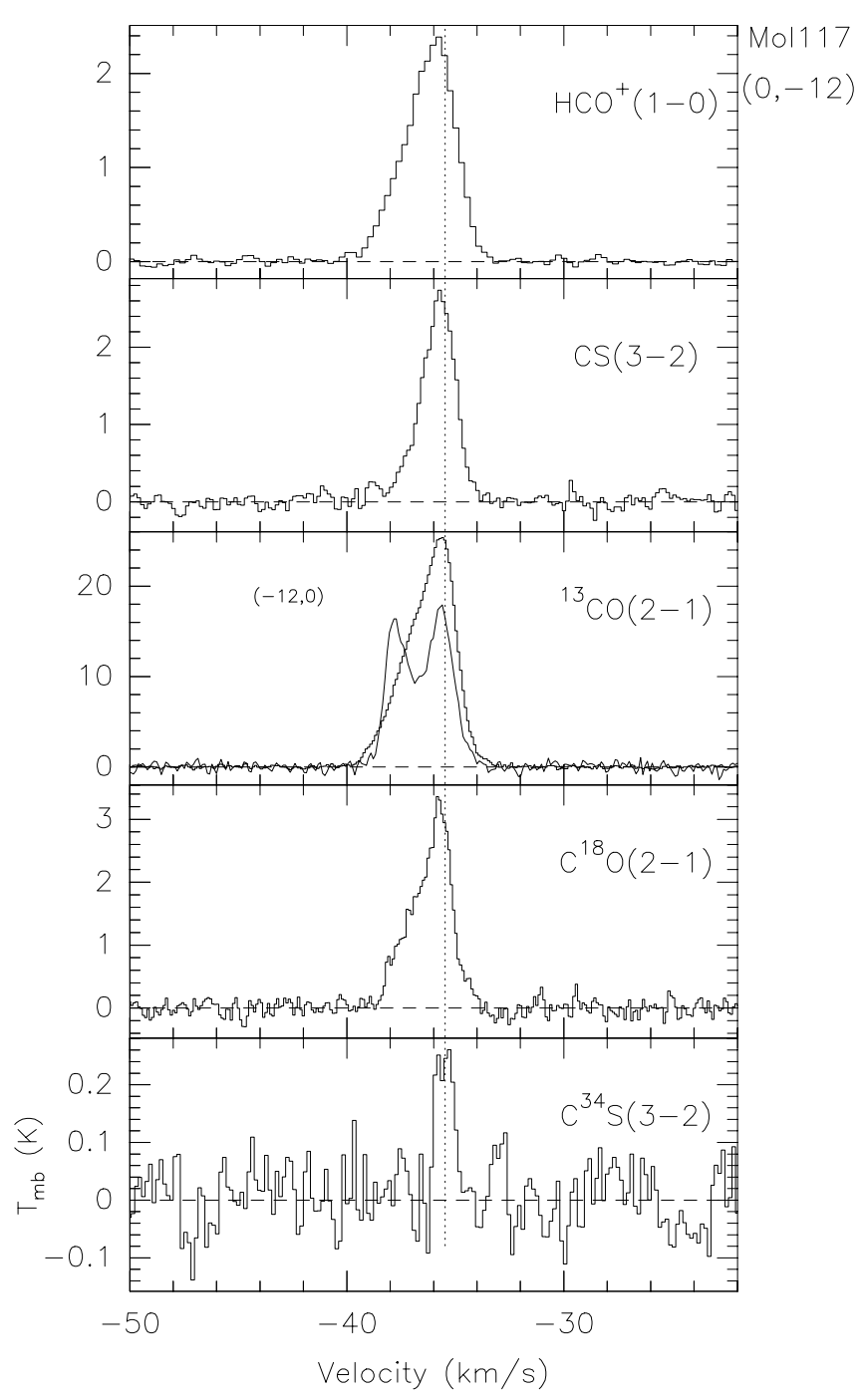

Fig. 1. g) As a), for Mol 117. The ${ }^{13} \mathrm{CO}(2-1)$ spectrum at $\left(12^{\prime \prime}, 0^{\prime \prime}\right)$ is also shown (drawn), for comparison

Focus, calibration, and receiver alignment were checked by observations of Venus; the alignment was within $2^{\prime \prime}$. During the observations of the program sources, pointing and calibration were checked by observations of well-known UC HII regions and continuum sources; the rms pointing accuracy was found to be $3^{\prime \prime}$, while line intensities were reproducible within 10\%-20\%. All line intensities in this paper are on a main beam brightness temperature $\left(T_{\mathrm{mb}}\right)$ scale.

Our sample of sources is listed in Table 2. Column 1 gives the source number from the list of Molinari et al. (1996); Cols. 2 and 3 give the equatorial (B1950) coordinates of the central position of the maps $(\approx$ the position of the sub-mm peak as found by Molinari et al. 2000); Cols. 4 and 5 give the galactic coordinates of the IRAS source, the name of which is in Col. 6; Col. 7 lists the radial velocity of the $\mathrm{NH}_{3}(1,1)$ main line (from Molinari et al. 1996). In Col. 8 we give the kinematic distances (from Molinari et al. 2000, 1996), while the luminosity, derived from the 


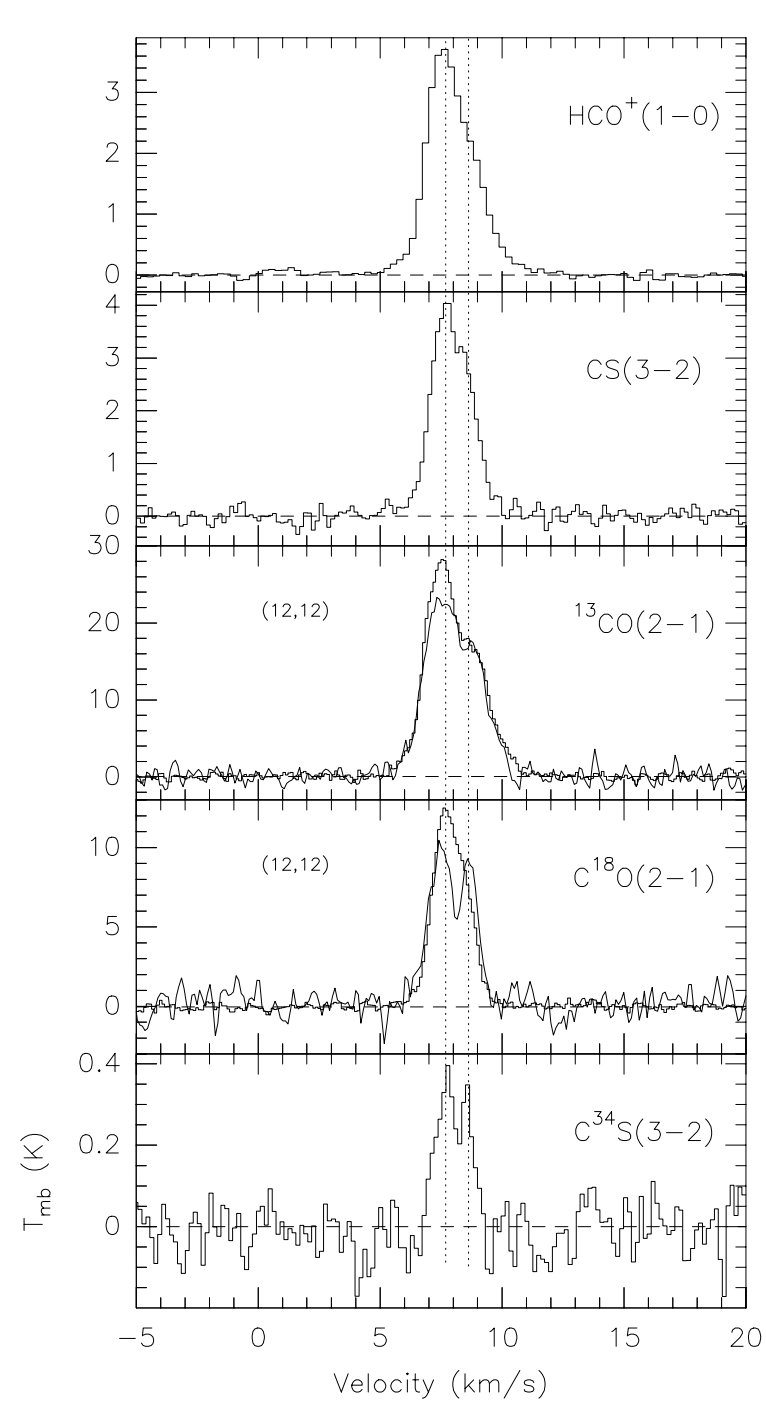

Fig. 1. h) As a), for Mol 118. The ${ }^{13} \mathrm{CO}(2-1)$ and $\mathrm{C}^{18} \mathrm{O}(2-1)$ spectra at $\left(12^{\prime \prime}, 12^{\prime \prime}\right)$ are also shown (drawn), for comparison. The dotted line with the red-shifted velocity, with respect to the $V_{\mathrm{lsr}}$ of the $\mathrm{C}^{34} \mathrm{~S}$ line, indicates the second component present in these observations (see Sect. 5)

IRAS fluxes, and (when available) from (sub-)mm data, is given in Col. 9. In Cols. 10 to 12 we present information on the presence/absence of $\mathrm{H}_{2} \mathrm{O}$ maser emission (from Palla et al. 1991), radio continuum (Molinari et al. 1996), and a mm-detected compact core (Molinari et al. 2000).

We started by making small maps around the sub-mm peak position in (simultaneously) $\mathrm{HCO}^{+},{ }^{13} \mathrm{CO}$, and CS. The initial grid size was $24^{\prime \prime}$, after which we zoomed in on the peak position on a $12^{\prime \prime}$ grid size. The maps were repeated several times, at least in the inner parts, in order to get a good signal-to-noise ratio. Total map extent was always $<100^{\prime \prime}$ in either direction. After having identified the peak position in this way, $\mathrm{C}^{18} \mathrm{O}, \mathrm{C}^{34} \mathrm{~S}, \mathrm{CH}_{3} \mathrm{C}_{2} \mathrm{H}$, and $\mathrm{CH}_{3} \mathrm{CN}$ were observed on a $3 \times 3$ grid, with step size $12^{\prime \prime}$, around the peak. The peak position itself was observed for up to $60 \mathrm{~min}$ in $\mathrm{CH}_{3} \mathrm{C}_{2} \mathrm{H}$ and $\mathrm{CH}_{3} \mathrm{CN}$. Observations were primarily done in total power mode, with an offset

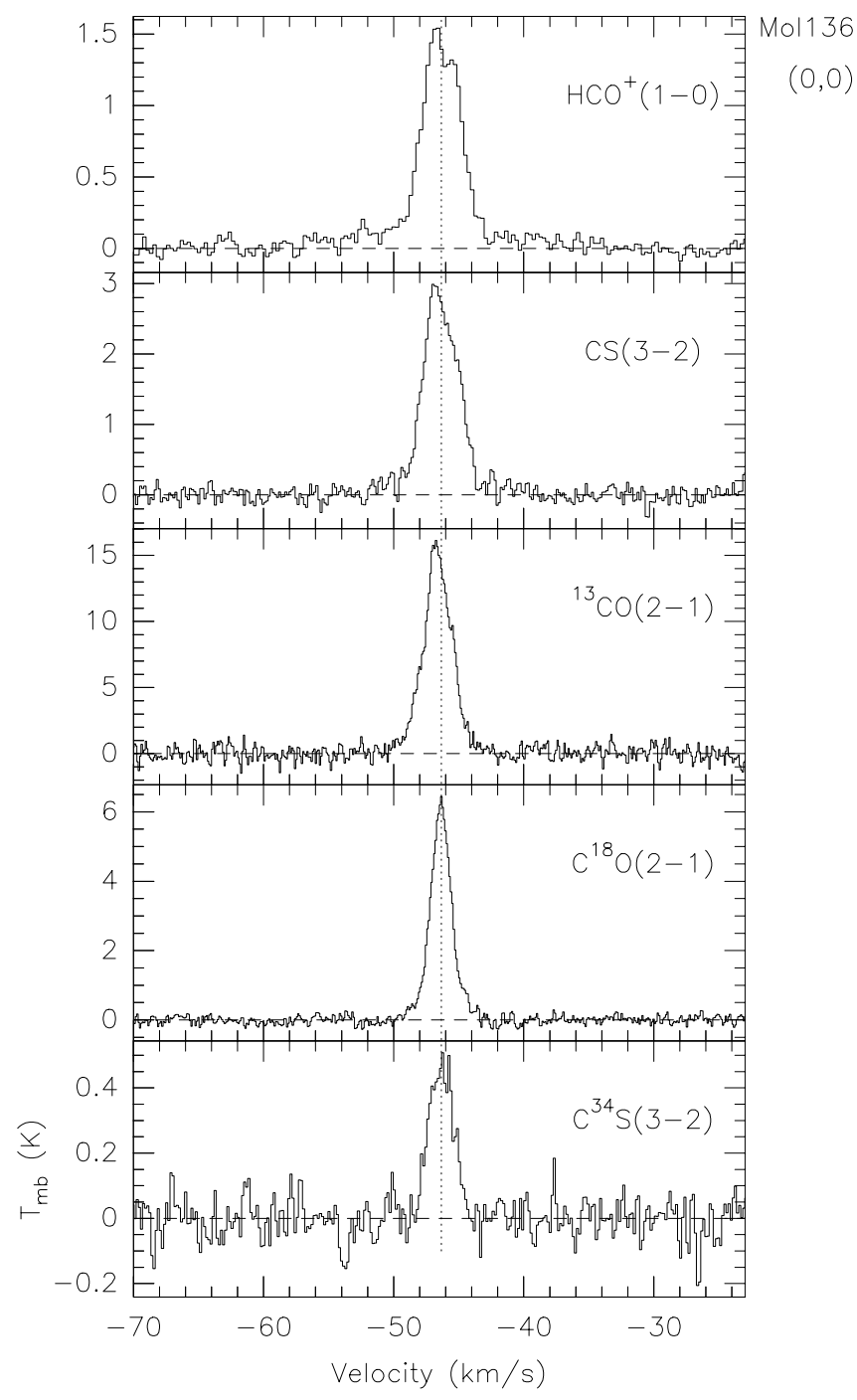

Fig. 1. i) As a), for Mol 136

position $1800^{\prime \prime}$ to the $\mathrm{W}$; observations of $\mathrm{CH}_{3} \mathrm{C}_{2} \mathrm{H}$ and $\mathrm{CH}_{3} \mathrm{CN}$ were done in wobbler mode, where the secondary mirror was offset by $240^{\prime \prime}$.

\subsection{KOSMA}

In April 1999, 6 sources from Table 2 were searched for $\mathrm{HCO}^{+}(4-3)$ emission $(\nu=356734.253 \mathrm{MHz})$ with the 3-m KOSMA telescope at Gornergrat. A liquid He-cooled SIS receiver was used, and the AOS backend provided a resolution of $0.14 \mathrm{~km} \mathrm{~s}^{-1}$. Single-pointing observations were made at the peak positions previously identified at IRAM. The KOSMA beam size was $\sim 70^{\prime \prime} \times 78^{\prime \prime}$; the beam efficiency was 0.75 . Each position was observed for 15 to $30 \mathrm{~min}$, resulting in an rms $\left(T_{\mathrm{mb}}\right)$ of $0.05-0.08 \mathrm{~K}$. The spectra suffer from standing waves, and are therefore of rather poor quality. 


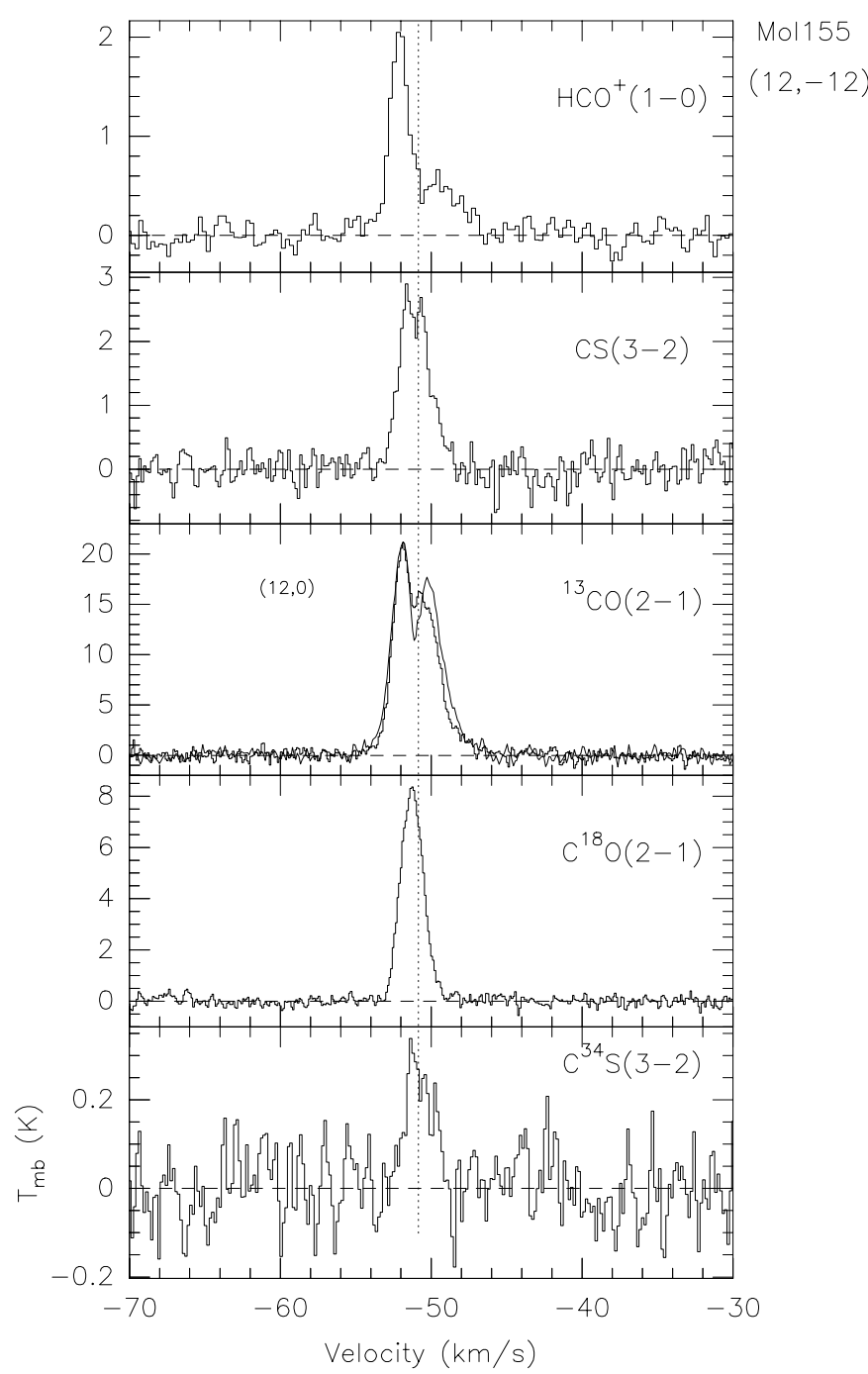

Fig. 1. j) As a), for Mol 155. The ${ }^{13} \mathrm{CO}(2-1)$ spectrum at $\left(12^{\prime \prime}, 0^{\prime \prime}\right)$ is also shown (drawn), for comparison

\section{Presentation of the data}

In this section a general presentation of the available data is made, before discussing the individual sources in more detail in Sect. 5. Because of the large amount of data, a selection had to be made. In the following, we try to show a representative sample of the available observations; individual spectra and maps can be made available upon request ${ }^{1}$.

\subsection{Spectra at the peak positions}

The spectra of $\mathrm{HCO}^{+}, \mathrm{CS},{ }^{13} \mathrm{CO}, \mathrm{C}^{18} \mathrm{O}$ and $\mathrm{C}^{34} \mathrm{~S}$, taken at the peak position, are shown in Figs. 1a-k. The relevant parameters of these lines are collected in Table 3. In this table we give the following information: in Col. 1

\footnotetext{
1 Contact J. Brand.
}

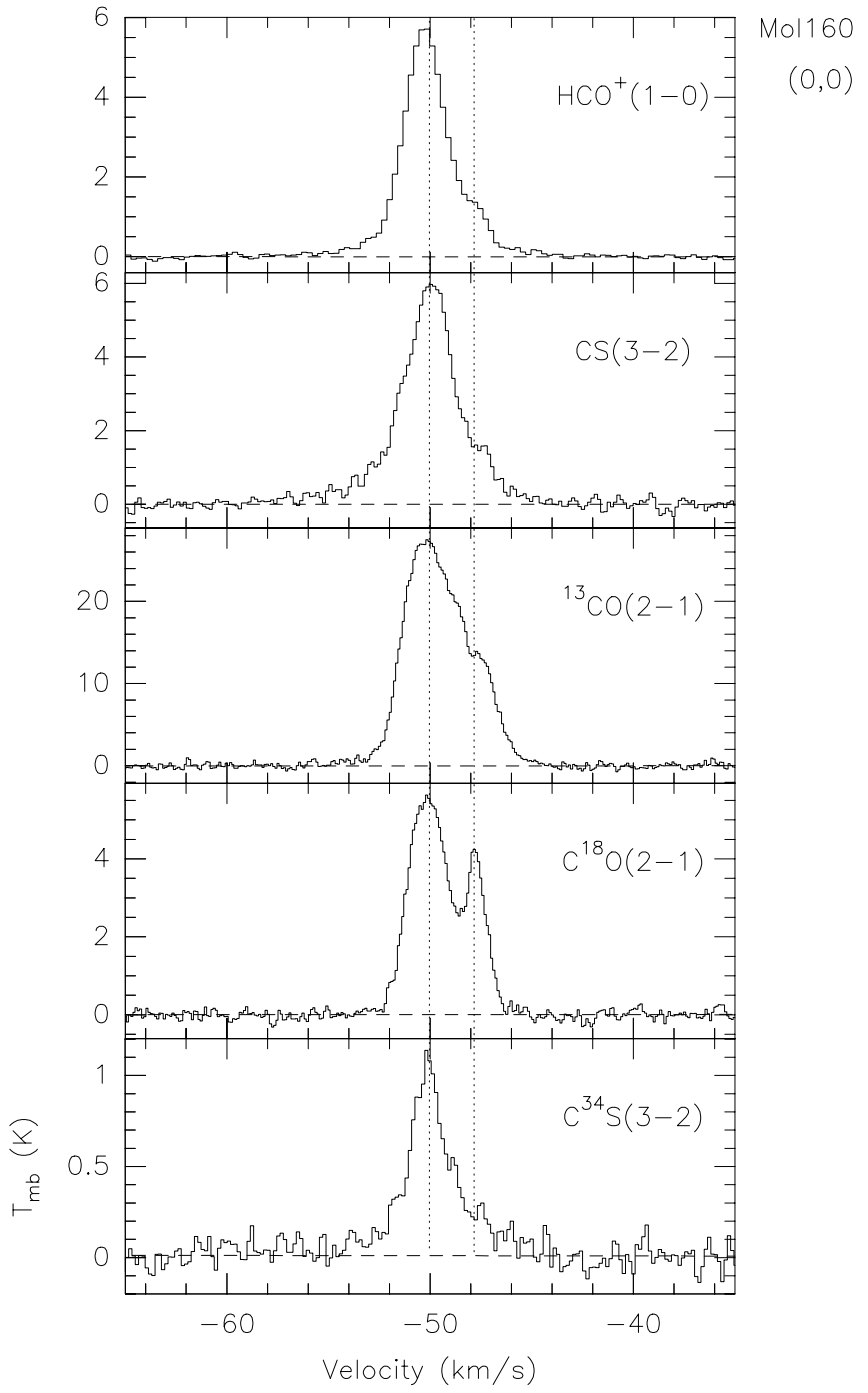

Fig. 1. k) As a), for Mol 160. The dotted line with the redshifted velocity, with respect to the $V_{\text {lsr }}$ of the $\mathrm{C}^{34} \mathrm{~S}$ line, indicates the second component present in these observations (see Sect. 5)

the molecular transition; in Col. 2 the velocity resolution of the spectrum; the rms noise of the spectrum in Col. 3; in Cols. 4 and 5 the extreme velocities $V_{\min }, V_{\max }$ where the line intensity drops below the $2 \sigma$ level; in Cols. 6,7 , and 8 the peak temperature, the velocity of the peak, and the FWHM line width, respectively. Because of the nonGaussian nature of many of the line profiles, these values are read off from the spectra (as opposed to being determined from Gaussian fits); in Cols. 9 and 10 the values of the integral over the line, between $V_{\min }$ and $V_{\max }(\mathrm{Col} .9)$, and between the velocities where $T_{\mathrm{mb}}=0 \mathrm{~K}$.

A comparison between the (IRAM) $\mathrm{HCO}^{+}(1-0)$ and the (KOSMA) $\mathrm{HCO}^{+}(4-3)$ emission is shown in Figs. 2a, b. The IRAM spectra were convolved to a $70^{\prime \prime}$ beam.

The spectra of the $\mathrm{CH}_{3} \mathrm{C}_{2} \mathrm{H}(6-5)$ and $\mathrm{CH}_{3} \mathrm{CN}(8-7)$ detections are presented in Figs. 3a-d and 4, respectively. Gaussfit parameters of these lines can be found in Tables 4 
Table 2. Observed sources

\begin{tabular}{|c|c|c|c|c|c|c|c|c|c|c|c|}
\hline $\begin{array}{l}\text { (1) } \\
\mathrm{Mol} \\
\# \\
\end{array}$ & $\begin{array}{c}(2) \\
\alpha(1950)^{\star} \\
(\mathrm{h} \mathrm{m} \mathrm{s})\end{array}$ & $\begin{array}{c}(3) \\
\delta(1950)^{\star} \\
(\circ \quad \prime \prime \prime) \\
\end{array}$ & $\begin{array}{c}(4) \\
l \\
\left({ }^{\circ}\right) \\
\end{array}$ & $\begin{array}{c}(5) \\
b \\
\left({ }^{\circ}\right) \\
\end{array}$ & $\begin{array}{c}(6) \\
\text { IRAS }\end{array}$ & $\begin{array}{c}(7) \\
V_{\mathrm{lsr}}\left(\mathrm{NH}_{3}\right) \\
\left(\mathrm{km} \mathrm{s}^{-1}\right) \\
\end{array}$ & $\begin{array}{c}(8) \\
\mathrm{d}_{\mathrm{kin}} \\
(\mathrm{kpc}) \\
\end{array}$ & $\begin{array}{c}(9) \\
L_{\mathrm{fir}} \\
\left(10^{4} L_{\odot}\right) \\
\end{array}$ & $\begin{array}{l}(10) \\
\mathrm{H}_{2} \mathrm{O}\end{array}$ & $\begin{array}{l}(11) \\
\text { Radio }^{1}\end{array}$ & $\begin{array}{l}(12) \\
\mathrm{mm}^{2}\end{array}$ \\
\hline+3 & 004206.3 & +553050 & 122.015 & -7.072 & $00420+5530$ & -51.2 & 7.7 & 5.15 & $\mathrm{Y}$ & $\mathrm{N}^{3}$ & Npp \\
\hline 8 & 051346.0 & +391910 & 168.061 & +0.821 & $05137+3919$ & -25.4 & 10.8 & $3.93^{5}$ & $\mathrm{Y}$ & $\mathrm{N}^{3}$ & $\mathrm{Y}$ \\
\hline 59 & 182749.7 & -100919 & 21.561 & -0.030 & $18278-1009$ & +93.7 & ${ }^{\dagger} 6.6$ & $1.46^{5,6}$ & $\mathrm{Y}$ & $\mathrm{N}$ & $\mathrm{Y}$ \\
\hline 75 & 185106.4 & +014640 & 34.821 & +0.351 & $18511+0146$ & +56.8 & 3.9 & $1.30^{5}$ & $\mathrm{~N}$ & $\mathrm{U}$ & $\mathrm{Y}$ \\
\hline 77 & 185246.2 & +030113 & 36.115 & +0.554 & $18527+0301$ & +76.0 & 5.3 & $0.90^{5}$ & $\mathrm{~N}$ & $\mathrm{~N}$ & $\mathrm{Y}$ \\
\hline 98 & 190913.0 & +084127 & 43.035 & -0.447 & $19092+0841$ & +58.0 & 4.5 & $0.92^{5}$ & $\mathrm{Y}$ & $\mathrm{U}^{3}$ & $\mathrm{Y}$ \\
\hline * 117 & 200954.3 & +364037 & 74.161 & +1.644 & $20099+3640$ & -36.4 & 8.7 & $2.51^{5}$ & $\mathrm{~N}$ & $\mathrm{Y}$ & $\mathrm{Y}$ \\
\hline * 118 & 201038.3 & +354542 & 73.479 & +1.016 & $20106+3545$ & +7.8 & 1.6 & 0.18 & $\mathrm{~N}$ & $\mathrm{~N}$ & Npp \\
\hline 136 & 213047.1 & +504901 & 94.262 & -0.411 & $21307+5049$ & -46.7 & 6.2 & $1.16^{5}$ & $\mathrm{Y}$ & $\mathrm{U}^{3}$ & $\mathrm{Y}$ \\
\hline 155 & 231403.4 & +612117 & 111.871 & +0.820 & $23140+6121$ & -51.5 & 5.2 & $1.06^{5}$ & $\mathrm{~N}^{4}$ & $\mathrm{Y}$ & $\mathrm{Y}$ \\
\hline * 160 & 233831.2 & +605343 & 114.531 & -0.543 & $23385+6053$ & -50.0 & 4.9 & $1.60^{5}$ & $\mathrm{Y}$ & $\mathrm{N}$ & $\mathrm{Y}$ \\
\hline
\end{tabular}

- map center (sub-mm peak Molinari et al. 2000).

* peak position also observed in $\mathrm{HCO}^{+}(4-3)$; see Sect. 3 .

$\dagger$ Derived from the present data; see Sect. 5 .

${ }^{1} \mathrm{U}=$ unassociated radio source is within $3^{\prime}$ radius.

${ }^{2}$ Compact mm-emission; Npp = Detected, but missed primary peak; see Molinari et al. (2000).

${ }^{3}$ Recently detected with the VLA-D array at 3.6-cm (see Molinari et al. 2000); detected signal is compatible with the non-detections at 2 and 6-cm reported in Molinari et al. (1998a).

${ }^{4}$ Maser discovered by Valdettaro et al. (2001).

${ }^{5}$ Luminosity from Molinari et al. (2000).

${ }^{6}$ Corrected for revised distance.

and 5. When making the Gaussian fits, we fixed the velocity separation of the various K-components, and forced the widths of all K-components in a spectrum to have the same value. In none of the sources the other transitions of $\mathrm{CH}_{3} \mathrm{CN}$ were detected, nor the $\mathrm{CH}_{3} \mathrm{OH}\left(v_{\mathrm{t}}=1\right)$ lines, which happen to lie close to the $\mathrm{C}^{34} \mathrm{~S}$ line.

Density-tracers, such as $\mathrm{CS}$ and the rare isotope $\mathrm{C}^{34} \mathrm{~S}$, have been detected in all sources of our sample, implying that dense cores are indeed present. The $\mathrm{C}^{34} \mathrm{~S}$ lines vary in strength from a few tenths of a $\mathrm{K}$, to more than $1 \mathrm{~K}$; only in Mol 3 was it barely detected.

\subsection{Distribution of the integrated emission}

The distributions of the integrated intensities of the emission of $\mathrm{HCO}^{+}(1-0),{ }^{13} \mathrm{CO}(2-1), \mathrm{CS}(3-2), \mathrm{C}^{18} \mathrm{O}(2-1)$, $\mathrm{C}^{34} \mathrm{~S}(3-2)$ (where strong enough) and $\mathrm{CH}_{3} \mathrm{C}_{2} \mathrm{H}(6-5)$ (for Mol 98; the only source where it has been detected at more than one position) are shown in Figs. 5a-k. For easy reference we have shown in all maps the location of the IRAS source (not always coinciding with the map center), and the location of the peak position at which longer integrations were made. From these distributions we derived the (beam-corrected) source sizes, and a mass estimate of the molecular cores; the results are collected in Table 6 . In this Table, Col. 1 gives the source name, Col. 2 the kinematic distance. In Cols. 3 to 6 we give the core size (in arcseconds and parsec), as determined from the transitions listed in the column headers. The sizes given here are those of the diameter of a circle with the same area as that enclosed by the FWHM contours in Figs. 5a-k. If the observed angular diameter is $\theta_{\mathrm{obs}}$, and the beam size at $F W H P$ is $\theta_{\text {beam }}$, then the beam-corrected size listed in the table is $\theta_{\text {cor }}=\sqrt{\theta_{\text {obs }}^{2}-\theta_{\text {beam }}^{2}}$. Finally, in Col. 7 we give a mass estimate of the core, as determined from the ${ }^{13} \mathrm{CO}$ observations. We note that this mass represents all emission above the FWHM contour in Figs. 5a-k, regardless of whether all this emission is associated with the embedded YSO. For this latter information, and for the assumptions made in the mass estimate, we refer to Sect. 5 . The mass estimates are lower limits, because only emission above the $50 \%$ level was used, and the FWHM contour is usually not closed inside the mapped area. The mass range is an order of magnitude, from $130 M_{\odot}(\mathrm{Mol} 118)$ to $2700 M_{\odot}(\mathrm{Mol} 3)$.

The $F W H M$ contour of the ${ }^{13} \mathrm{CO}$ emission extends beyond the limits of the maps in all but two of the observed sources. For the $\mathrm{HCO}^{+}$emission we find the same, for most sources. Only the CS emission is well within the map boundaries, except for Mol 155, but even there the FWHM contour is almost closed inside the mapped region (Fig. 5j). Unfortunately the maps in $\mathrm{C}^{18} \mathrm{O}$ and $\mathrm{C}^{34} \mathrm{~S}$ are too small to allow a size determination.

Figure 5 shows that the peak position of the higherdensity tracers does not always coincide with that of the mm-continuum peak $\left(=0^{\prime \prime}, 0^{\prime \prime}\right)$. However, for those sources for which new SCUBA observations (at $450 \mu \mathrm{m}$ and/or $850 \mu \mathrm{m}$; Molinari et al., in preparation) are available, we find that the correspondence between molecularand sub-mm-peak is good. The reason is that Molinari et al. (2000) determined the location of the mm-peak from 5 -point cross observations in most cases, rather than from 
Table 3. Line parameters at peak position

\begin{tabular}{|c|c|c|c|c|c|c|c|c|c|}
\hline Line & $\begin{array}{c}V_{\text {res }} \\
\left(\mathrm{kms}^{-1}\right)\end{array}$ & $\begin{array}{l}\mathrm{rms} \\
(\mathrm{K}) \\
\end{array}$ & $\begin{array}{c}V_{\min } \\
\left(\mathrm{km} \mathrm{s}^{-1}\right)\end{array}$ & $\begin{array}{c}V_{\max } \\
\left(\mathrm{km} \mathrm{s}^{-1}\right)\end{array}$ & $\begin{array}{r}T_{\mathrm{mb}} \\
(\mathrm{K}) \\
\end{array}$ & $\begin{array}{c}V_{\mathrm{lsr}} \\
\left(\mathrm{km} \mathrm{s}^{-1}\right)\end{array}$ & $\begin{array}{c}\Delta v \\
\left(\mathrm{~km} \mathrm{~s}^{-1}\right)\end{array}$ & $\begin{array}{r}\int T_{\mathrm{mb}} \mathrm{d} v^{1} \\
\left(\mathrm{Kkm} \mathrm{s}^{-1}\right) \\
\end{array}$ & $\underset{\left(\mathrm{Kkm} \mathrm{s}^{-1}\right)}{\int T_{\mathrm{mb}} \mathrm{d} v^{2}}$ \\
\hline \multicolumn{10}{|c|}{$\operatorname{Mol} 3\left(0^{\prime \prime},-24^{\prime \prime}\right)$} \\
\hline $\mathrm{HCO}^{+}(1-0)$ & 0.26 & 0.045 & -53.8 & -48.3 & 2.4 & -51.9 & $\begin{array}{l}1.3 \\
3.2\end{array}$ & 6.2 & 6.6 \\
\hline${ }^{13} \mathrm{CO}(2-1)$ & 0.11 & 0.78 & -53.2 & -48.4 & 27.0 & -52.0 & $\begin{array}{c}1.5 \\
3.1^{3}\end{array}$ & 67.3 & 68.8 \\
\hline $\mathrm{CS}(3-2)$ & 0.16 & 0.12 & -53.2 & -48.5 & 2.1 & -50.8 & 2.7 & 5.7 & 5.8 \\
\hline $\mathrm{C}^{18} \mathrm{O}(2-1)$ & 0.11 & 0.16 & -52.8 & -49.0 & 6.7 & -50.3 & 2.7 & 16.6 & 16.9 \\
\hline $\mathrm{C}^{34} \mathrm{~S}(3-2)$ & 0.16 & 0.073 & & & & & & & \\
\hline smoothed: & 0.65 & 0.039 & -52.0 & -49.4 & 0.1 & -50.4 & 2.6 & 0.3 & 0.4 \\
\hline \multicolumn{10}{|c|}{$\operatorname{Mol} 8\left(0^{\prime \prime}, 0^{\prime \prime}\right)$} \\
\hline $\mathrm{HCO}^{+}(1-0)$ & 0.26 & 0.082 & -30.7 & -21.5 & 1.3 & -25.3 & 3.4 & 5.3 & 6.4 \\
\hline${ }^{13} \mathrm{CO}(2-1)$ & 0.11 & 0.25 & -29.6 & -20.3 & 16.6 & -25.4 & 2.8 & 56.6 & 59.4 \\
\hline $\mathrm{CS}(3-2)$ & 0.16 & 0.16 & -27.8 & -22.7 & 4.1 & -25.1 & 2.1 & 10.3 & 10.5 \\
\hline $\mathrm{C}^{18} \mathrm{O}(2-1)$ & 0.11 & 0.14 & -27.3 & -22.6 & 3.5 & -25.3 & 2.1 & 7.7 & 7.9 \\
\hline $\mathrm{C}^{34} \mathrm{~S}(3-2)$ & 0.16 & 0.079 & -26.3 & -23.9 & 1.2 & -25.1 & 1.3 & 1.7 & 2.0 \\
\hline \multicolumn{10}{|c|}{ Mol $59\left(12^{\prime \prime}, 0^{\prime \prime}\right)$} \\
\hline $\mathrm{HCO}^{+}(1-0)$ & 0.26 & 0.11 & 111.8 & 118.9 & 1.4 & 115.5 & $\begin{array}{c}1.8 \\
4.2^{3}\end{array}$ & 4.1 & 4.2 \\
\hline${ }^{13} \mathrm{CO}(2-1)$ & 0.11 & 0.49 & 107.2 & 120.1 & 9.1 & 115.1 & 5.5 & 58.3 & 61.3 \\
\hline $\mathrm{CS}(3-2)$ & 0.16 & 0.32 & 112.3 & 115.8 & 2.4 & 114.8 & 2.9 & 5.4 & 6.8 \\
\hline $\mathrm{C}^{18} \mathrm{O}(2-1)$ & 0.11 & 0.25 & 111.0 & 117.1 & 4.9 & 113.4 & 2.0 & 13.3 & 15.8 \\
\hline $\mathrm{C}^{34} \mathrm{~S}(3-2)$ & 0.16 & 0.12 & 113.6 & 115.1 & 0.4 & 113.9 & 1.9 & 0.5 & 0.8 \\
\hline \multicolumn{10}{|c|}{ Mol $75\left(0^{\prime \prime}, 0^{\prime \prime}\right)$} \\
\hline $\mathrm{HCO}^{+}(1-0)$ & 0.26 & 0.069 & 54.7 & 69.1 & 1.6 & 59.6 & $\begin{array}{c}1.8 \\
2.9^{3}\end{array}$ & 7.4 & 9.8 \\
\hline${ }^{13} \mathrm{CO}(2-1)$ & 0.11 & 0.55 & 50.5 & 61.3 & 11.5 & 57.0 & 4.4 & 59.2 & 65.3 \\
\hline $\mathrm{CS}(3-2)$ & 0.16 & 0.20 & 53.6 & 61.2 & 5.0 & 56.8 & 2.9 & 17.7 & 17.8 \\
\hline $\mathrm{C}^{18} \mathrm{O}(2-1)$ & 0.11 & 0.17 & 53.0 & 60.4 & 8.1 & 57.3 & 3.3 & 27.9 & 28.3 \\
\hline $\mathrm{C}^{34} \mathrm{~S}(3-2)$ & 0.16 & 0.094 & 56.2 & 59.3 & 0.7 & 57.0 & 1.9 & 1.4 & 1.6 \\
\hline \multicolumn{10}{|c|}{ Mol $77\left(0^{\prime \prime}, 0^{\prime \prime}\right)$} \\
\hline $\mathrm{HCO}^{+}(1-0)$ & 0.26 & 0.090 & 72.1 & 79.1 & 1.4 & 75.2 & 1.8 & 4.0 & 4.3 \\
\hline${ }^{13} \mathrm{CO}(2-1)$ & 0.11 & 0.35 & 71.9 & 82.1 & 10.1 & 75.1 & 4.2 & 42.5 & 42.9 \\
\hline $\mathrm{CS}(3-2)$ & 0.16 & 0.25 & 72.8 & 78.0 & 2.6 & 75.5 & 2.9 & 7.4 & 7.8 \\
\hline $\mathrm{C}^{18} \mathrm{O}(2-1)$ & 0.11 & 0.22 & 73.8 & 78.3 & 8.5 & 75.7 & 2.4 & 20.8 & 21.1 \\
\hline $\mathrm{C}^{34} \mathrm{~S}(3-2)$ & 0.16 & 0.073 & 74.1 & 77.5 & 1.0 & 76.4 & 1.6 & 1.9 & 2.4 \\
\hline
\end{tabular}

\footnotetext{
${ }^{1}$ Integral taken between $V_{\min }$ and $V_{\max }$.

${ }^{2}$ Integral taken between velocities where $T_{\mathrm{mb}}=0 \mathrm{~K}$.

${ }^{3}$ In line profiles with a "dip" which is $<0.5 T_{\text {peak }}$, the $\Delta v$ on the second line is the $F W H M$ taken over the whole line.
}

mapping, which may have resulted in a not very accurate determination of the mm-peak in some cases. The IRAS source is usually within a few arcseconds from the mm-peak position, with the exception of Mols $3\left(8^{\prime \prime} .7\right)$, $98\left(5^{\prime \prime} .9\right), 155\left(11^{\prime \prime} .9\right)$, and $160\left(8^{\prime \prime}\right)$. Considering the sizes of the IRAS point source error ellipses (see e.g. Molinari et al. 2000), these deviations are not significant.

\subsection{Line shapes}

At many positions in the maps, but certainly at the peak positions, the signal-to-noise of the spectra is high enough for a detailed look at the line shapes. The rare isotopomer
$\mathrm{C}^{34} \mathrm{~S}$, with its low abundance and relatively high critical density, is a tracer of the denser regions of molecular clouds. Its line profiles can be fitted with a single Gaussian, with only a few exceptions: Mol 118 and Mol 160, where there are two components (see Sect. 5). In Mol 77 and 98, at low emission levels, there are deviations from a Gaussian on the blue side (perhaps due to outflowing gas). Because of this, the velocity of $\mathrm{C}^{34} \mathrm{~S}$ can be assumed to represent the velocity of the high-density gas, and can be used to identify asymmetries in the line profiles of the other transitions observed here. For this purpose, in Figs. 6a-d we show the spectra of ${ }^{13} \mathrm{CO}(2-1)$, 
Table 3. continued

\begin{tabular}{|c|c|c|c|c|c|c|c|c|c|}
\hline Line & $\begin{array}{c}V_{\text {res }} \\
\left(\mathrm{km} \mathrm{s}^{-1}\right)\end{array}$ & $\begin{array}{l}\mathrm{rms} \\
(\mathrm{K})\end{array}$ & $\begin{array}{c}V_{\min } \\
\left(\mathrm{km} \mathrm{s}^{-1}\right)\end{array}$ & $\begin{array}{c}V_{\max } \\
\left(\mathrm{km} \mathrm{s}^{-1}\right) \\
\end{array}$ & $\begin{array}{r}T_{\mathrm{mb}} \\
(\mathrm{K}) \\
\end{array}$ & $\begin{array}{c}V_{\mathrm{lsr}} \\
\left(\mathrm{km} \mathrm{s}^{-1}\right)\end{array}$ & $\begin{array}{c}\Delta v \\
\left(\mathrm{~km} \mathrm{~s}^{-1}\right)\end{array}$ & $\underset{\left(\mathrm{Kkm} \mathrm{s}^{-1}\right)}{\int T_{\mathrm{mb}} \mathrm{d} v^{1}}$ & $\begin{array}{r}\int T_{\mathrm{mb}} \mathrm{d} v^{2} \\
\left(\mathrm{Kkm} \mathrm{s}^{-1}\right)\end{array}$ \\
\hline \multicolumn{10}{|c|}{ Mol $98\left(24^{\prime \prime}, 0^{\prime \prime}\right)$} \\
\hline $\mathrm{HCO}^{+}(1-0)$ & 0.26 & 0.041 & 45.1 & 61.4 & 2.4 & 56.4 & 5.7 & 14.8 & 15.0 \\
\hline${ }^{13} \mathrm{CO}(2-1)$ & 0.11 & 0.22 & 53.0 & 63.2 & 17.7 & 57.0 & 4.0 & 74.6 & 75.1 \\
\hline $\mathrm{CS}(3-2)$ & 0.16 & 0.096 & 52.2 & 63.8 & 3.1 & 56.9 & 6.1 & 18.9 & 20.2 \\
\hline $\mathrm{C}^{18} \mathrm{O}(2-1)$ & 0.11 & 0.13 & 53.7 & 62.3 & 9.0 & 58.3 & 3.6 & 33.7 & 33.9 \\
\hline $\mathrm{C}^{34} \mathrm{~S}(3-2)$ & 0.16 & 0.070 & 54.0 & 60.5 & 1.0 & 57.7 & 4.1 & 4.0 & 4.3 \\
\hline \multicolumn{10}{|c|}{$\operatorname{Mol} 117\left(0^{\prime \prime},-12^{\prime \prime}\right)$} \\
\hline $\mathrm{HCO}^{+}(1-0)$ & 0.26 & 0.034 & -40.1 & -33.5 & 2.4 & -35.8 & 2.6 & 6.7 & 6.8 \\
\hline${ }^{13} \mathrm{CO}(2-1)$ & 0.11 & 0.14 & -39.7 & -33.3 & 25.3 & -35.6 & 2.6 & 66.8 & 66.9 \\
\hline $\mathrm{CS}(3-2)$ & 0.16 & 0.085 & -38.1 & -34.1 & 2.7 & -35.7 & 1.8 & 5.1 & 5.4 \\
\hline $\mathrm{C}^{18} \mathrm{O}(2-1)$ & 0.11 & 0.12 & -38.3 & -34.2 & 3.3 & -35.8 & 1.8 & 6.6 & 6.7 \\
\hline $\mathrm{C}^{34} \mathrm{~S}(3-2)$ & 0.16 & 0.057 & -36.0 & -34.9 & 0.2 & -35.5 & 1.0 & 0.24 & 0.25 \\
\hline \multicolumn{10}{|c|}{ Mol $118\left(12^{\prime \prime}, 0^{\prime \prime}\right)$} \\
\hline $\mathrm{HCO}^{+}(1-0)$ & 0.26 & 0.048 & 5.2 & 11.2 & 3.7 & 7.7 & 2.4 & 8.6 & 8.7 \\
\hline${ }^{13} \mathrm{CO}(2-1)$ & 0.11 & 0.39 & 5.4 & 10.7 & 28.0 & 7.6 & 2.5 & 67.7 & 68.4 \\
\hline $\mathrm{CS}(3-2)$ & 0.16 & 0.13 & 6.2 & 10.0 & 4.0 & 7.7 & 1.9 & 7.7 & 7.9 \\
\hline $\mathrm{C}^{18} \mathrm{O}(2-1)$ & 0.11 & 0.19 & 6.2 & 9.6 & 12.4 & 7.7 & 1.7 & 21.2 & 21.5 \\
\hline $\mathrm{C}^{34} \mathrm{~S}(3-2)$ & 0.16 & 0.058 & 6.9 & 9.1 & 0.4 & 7.8 & 1.8 & 0.57 & 0.56 \\
\hline \multicolumn{10}{|c|}{ Mol $136\left(0^{\prime \prime}, 0^{\prime \prime}\right)$} \\
\hline $\mathrm{HCO}^{+}(1-0)$ & 0.26 & 0.041 & -52.8 & -43.0 & 1.5 & -46.7 & 3.4 & 5.8 & 6.4 \\
\hline${ }^{13} \mathrm{CO}(2-1)$ & 0.11 & 0.54 & -49.4 & -44.1 & 15.7 & -46.8 & 2.3 & 40.3 & 41.8 \\
\hline $\mathrm{CS}(3-2)$ & 0.16 & 0.098 & -49.6 & -43.7 & 3.0 & -46.8 & 3.2 & 9.3 & 10.1 \\
\hline $\mathrm{C}^{18} \mathrm{O}(2-1)$ & 0.11 & 0.11 & -49.1 & -43.4 & 6.3 & -46.3 & 1.8 & 12.7 & 12.7 \\
\hline $\mathrm{C}^{34} \mathrm{~S}(3-2)$ & 0.16 & 0.054 & -47.9 & -44.9 & 0.5 & -46.2 & 1.9 & 1.0 & 1.1 \\
\hline \multicolumn{10}{|c|}{ Mol $155\left(12^{\prime \prime},-12^{\prime \prime}\right)$} \\
\hline $\mathrm{HCO}^{+}(1-0)$ & 0.26 & 0.11 & -53.6 & -47.6 & 2.0 & -52.0 & 1.3 & 4.6 & 4.9 \\
\hline${ }^{13} \mathrm{CO}(2-1)$ & 0.11 & 0.47 & -53.6 & -47.2 & 20.9 & -51.9 & 3.1 & 61.5 & 62.2 \\
\hline $\mathrm{CS}(3-2)$ & 0.16 & 0.21 & -52.8 & -49.2 & 2.8 & -51.6 & 2.1 & 6.2 & 6.6 \\
\hline $\mathrm{C}^{18} \mathrm{O}(2-1)$ & 0.11 & 0.14 & -53.0 & -49.3 & 8.3 & -51.3 & 1.7 & 15.3 & 15.5 \\
\hline $\mathrm{C}^{34} \mathrm{~S}(3-2)$ & 0.16 & 0.075 & -51.6 & -50.1 & 0.3 & -51.4 & 1.5 & 0.38 & 0.61 \\
\hline \multicolumn{10}{|c|}{ Mol $160\left(0^{\prime \prime}, 0^{\prime \prime}\right)$} \\
\hline $\mathrm{HCO}^{+}(1-0)$ & 0.26 & 0.044 & -56.4 & -44.2 & 5.8 & -50.3 & 2.3 & 17.5 & 17.6 \\
\hline${ }^{13} \mathrm{CO}(2-1)$ & 0.11 & 0.32 & -54.8 & -44.8 & 27.0 & -50.2 & $\begin{array}{c}3.7 \\
4.0^{3}\end{array}$ & 107.2 & 107.7 \\
\hline $\mathrm{CS}(3-2)$ & 0.16 & 0.12 & -55.1 & -45.2 & 6.0 & -50.0 & 2.9 & 21.2 & 22.2 \\
\hline $\mathrm{C}^{18} \mathrm{O}(2-1)$ & 0.11 & 0.12 & -52.3 & -46.4 & 5.6 & -50.1 & 2.5 & 18.5 & 18.8 \\
\hline $\mathrm{C}^{34} \mathrm{~S}(3-2)$ & 0.16 & 0.065 & -52.1 & -47.0 & 1.1 & -50.1 & 2.0 & 2.6 & 3.0 \\
\hline
\end{tabular}

$\mathrm{HCO}^{+}(1-0), \mathrm{CS}(3-2)$, and $\mathrm{C}^{18} \mathrm{O}(2-1)$ aligned with the $\mathrm{C}^{34} \mathrm{~S}(3-2)$ velocity.

Figure 6a shows that none of the ${ }^{13} \mathrm{CO}$ profiles is a simple Gaussian, except perhaps for Mol 8, although broader emission is visible in the wings on both sides. Several of the profiles show dips, especially Mol 3, 59, and 77, while others (Mol 118, 155, 160) show shoulders. These deviations may be due to the superposition at that location of separate velocity components, or due to self-absorption. The latter can be produced either by an intervening colder cloud at the same velocity, or by a temperature gradient in the cloud itself. Profiles with stronger blue peaks can also be the signature of infalling gas (e.g. Myers et al. 2000), although it is unlikely that such a phenomenon is visible in the present observations, considering the large distances of the sources in our sample, and the relatively large beam sizes involved.

The asymmetry of the profiles can be quantified by looking at the ratio of $\int T \mathrm{~d} v$ on the blue- and red sides of the lines (where "blue" and "red" are relative to the $\mathrm{C}^{34} \mathrm{~S}$ velocity). The distribution of this ratio for each transition is shown in Fig. 7. The average ratio (indicated in each panel), is $>1$ for all tracers, indicating a clear blue asymmetry in the sample. The largest deviations from a Gaussian shape are found for the $\mathrm{HCO}^{+}$profiles (Fig. 6b). By contrast, the CS spectra are all more or less symmetric with respect to the $\mathrm{C}^{34} \mathrm{~S}$ velocity: deviations from Gaussian profiles are seen in the CS spectra for Mol 3, 77, and 98 , which are flat-topped, while there may be a dip in that for Mol 155. Mol 8, 59, 98, and 160 have ratios $\approx 1$. 


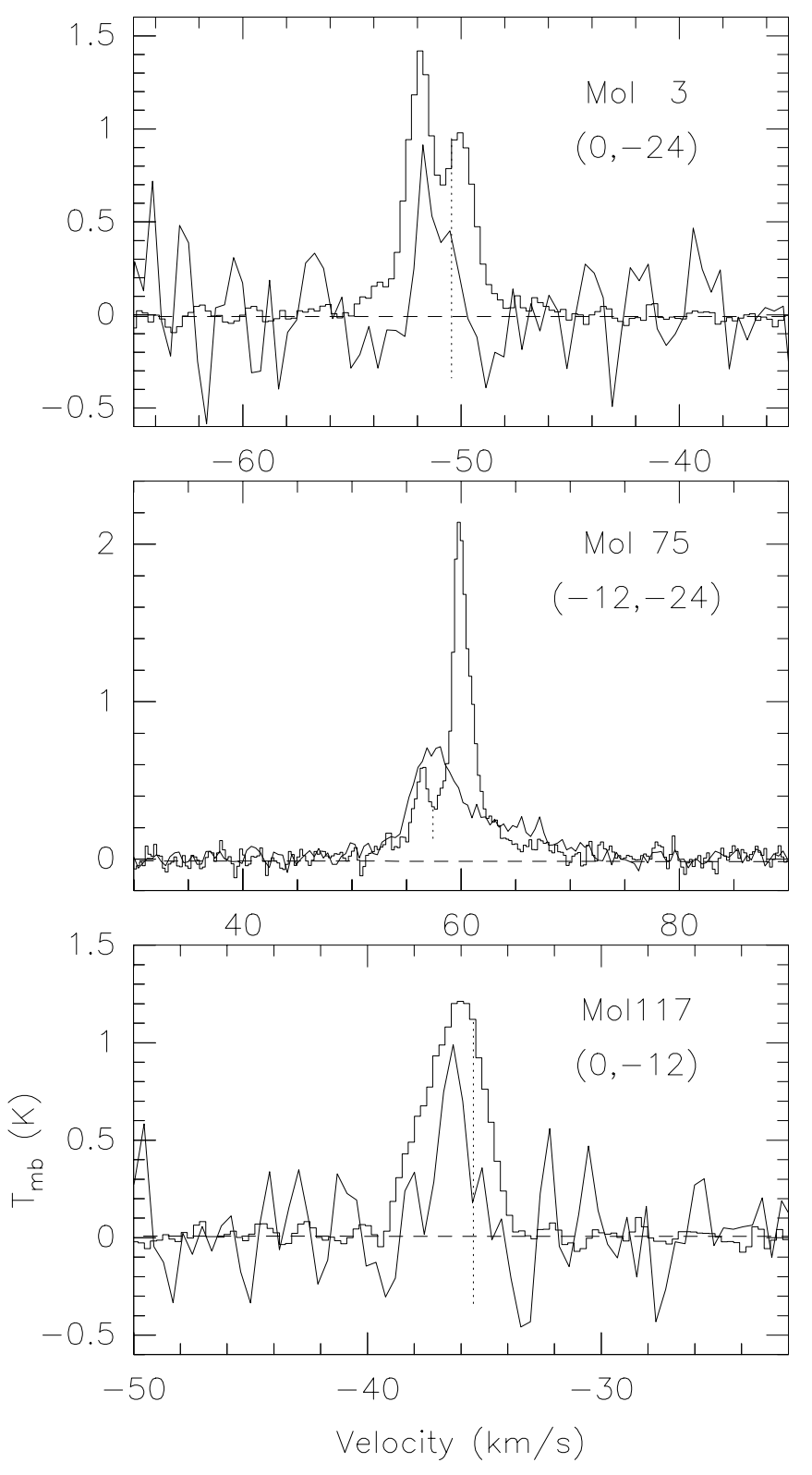

Fig. 2. a) Comparison of the IRAM $\mathrm{HCO}^{+}(1-0)$ [histogram] spectra with the KOSMA $\mathrm{HCO}^{+}(4-3)$ [drawn] spectra, at the indicated positions, for Mol 3, 75, and 117. The dotted vertical lines indicate the $V_{\text {lsr }}$ of the $\mathrm{C}^{34} \mathrm{~S}(3-2)$ emission (from Gaussian fits); the dashed horizontal lines indicate the $0 \mathrm{~K}$ level. The IRAM spectra were convolved to a $70^{\prime \prime}$ beam; the KOSMA spectra were smoothed to a resolution of $0.4 \mathrm{~km} \mathrm{~s}^{-1}$, and multiplied by 7 (Mol 3), and 5 (Mol 117) to make comparison easier

The $\mathrm{C}^{18} \mathrm{O}$ profiles are also more Gaussian in shape than those of ${ }^{13} \mathrm{CO}$ and $\mathrm{HCO}^{+}$, although less so than those of CS.

The interpretation of these numbers is not straightforward, because most spectra have multiple emission components (see Sect. 5), some of which may not be associated with the embedded IRAS sources (about half of the objects under investigation are located in the inner Galaxy). Some general remarks can be made, however. The range

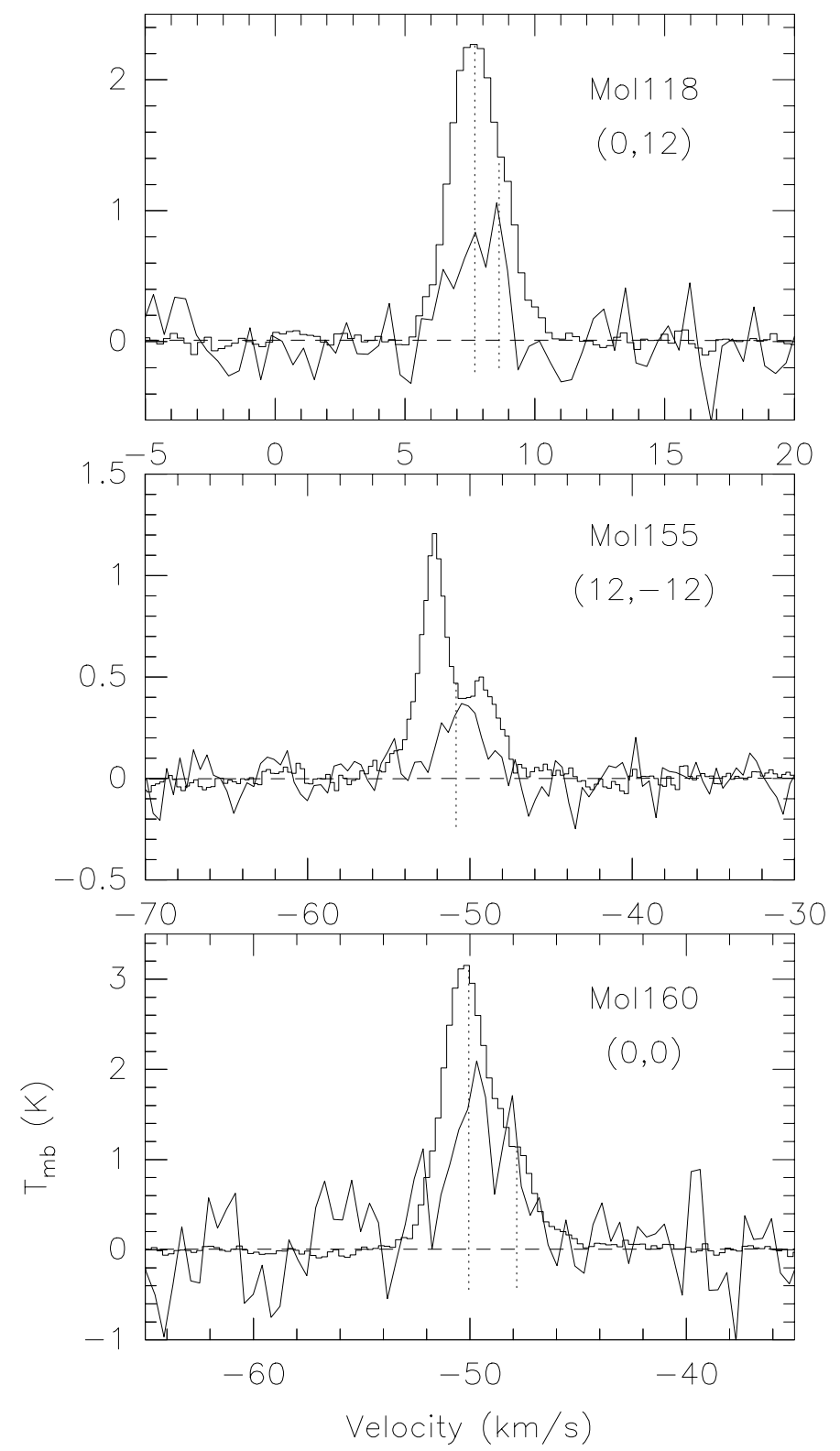

Fig. 2. b) As a), for Mol 118, 155, and 160. The KOSMA spectra were multiplied by 9 (Mol 118), 2 (Mol 155), and 10 (Mol 160), to make comparison easier

in the ratios for the various tracers is an indication for their kinematical behaviour. For instance, the profiles of the tracers of the lower-density gas (like ${ }^{13} \mathrm{CO}$ or $\mathrm{C}^{18} \mathrm{O}$ ) show evidence of a kinematical behaviour different from that of the higher-density gas (as represented by the $\mathrm{C}^{34} \mathrm{~S}$ lines). On the other hand, the higher-density tracers (CS) tend to have narrower profiles, which are more symmetric with respect to the velocity of the high-density gas. The profiles of transitions like ${ }^{13} \mathrm{CO}$, which sample the 


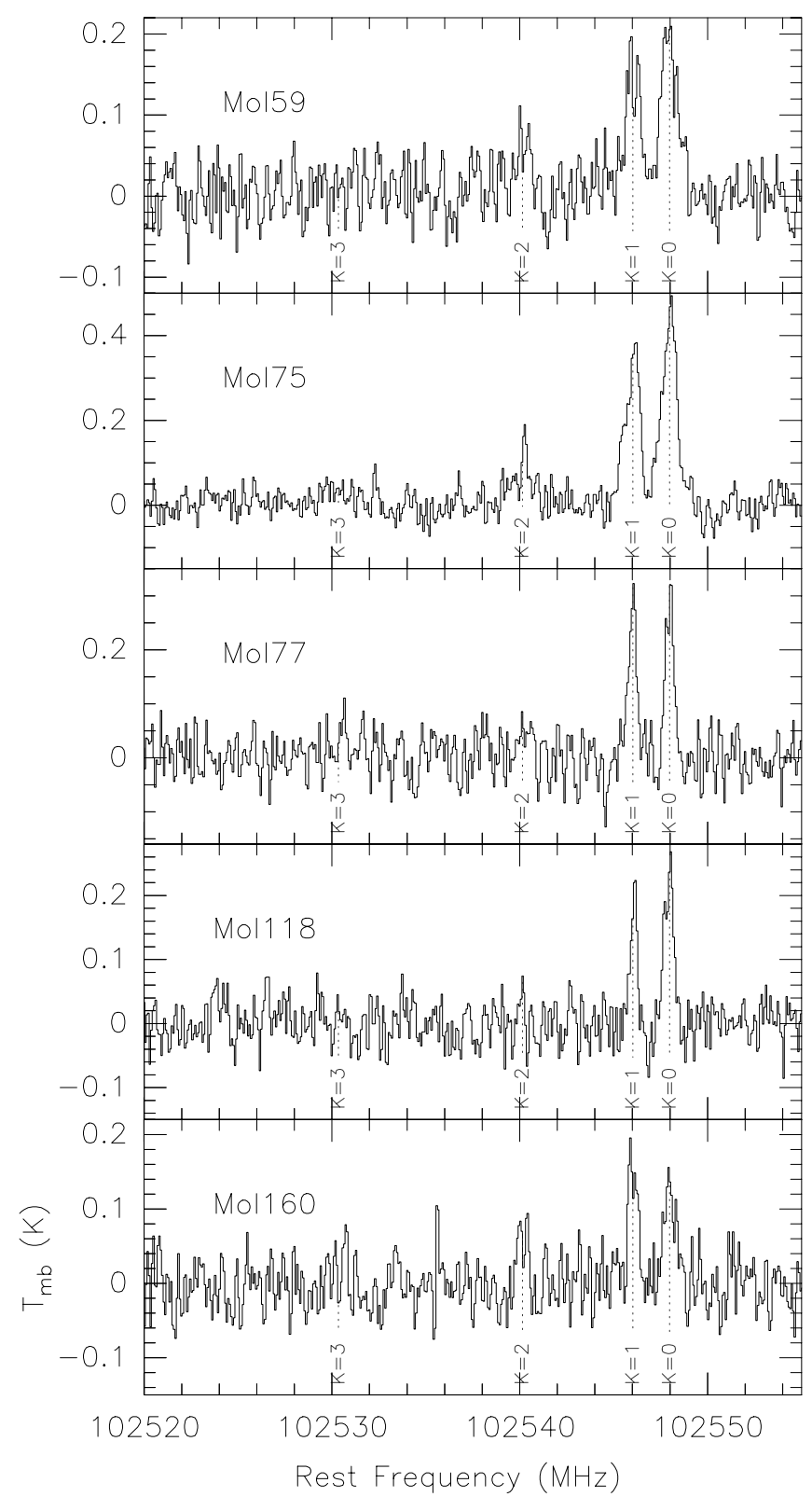

Fig. 3. a) Spectra of the $\mathrm{CH}_{3} \mathrm{C}_{2} \mathrm{H}(6-5)$ rotational transition towards the peak position. Only the higher-resolution $\left(0.23 \mathrm{~km} \mathrm{~s}^{-1}\right)$ spectra are shown. The location of the first few $\mathrm{K}$-components are indicated in the spectra

more diffuse gas in which the high-density clumps are embedded, are shaped by larger-scale turbulence and by the superposition of various velocity components, that are not directly related to the molecular core in which the YSO is embedded. The $\mathrm{HCO}^{+}$emission shows the largest average $\int T \mathrm{~d} v$ blue/red ratio, as well as the largest range; this may be related to the fact that $\mathrm{HCO}^{+}$is also produced in shocks, causing its kinematics to differ considerably from that of the $\mathrm{C}^{34} \mathrm{~S}$ gas.

The ratio of $\int T \mathrm{~d} v \quad \mathrm{CS} / \mathrm{C}^{34} \mathrm{~S}$ ranges between 4.7 (Mol 98) and 21.6 (Mol 117); the peak temperature ratio

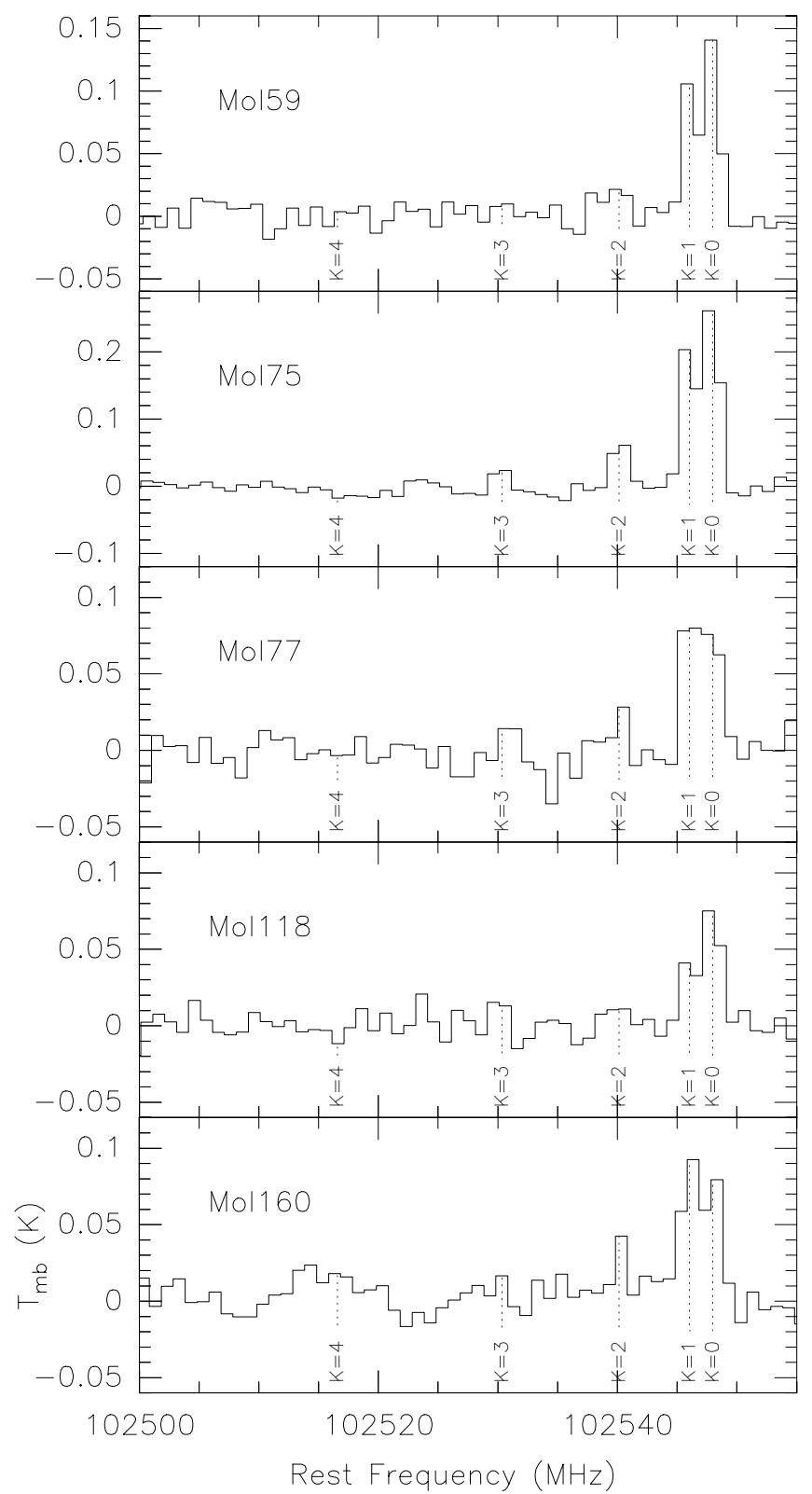

Fig. 3. b) As a), but for the low-resolution $\left(2.92 \mathrm{~km} \mathrm{~s}^{-1}\right)$ spectra

varies from $2.2(\mathrm{Mol} 77)$ to $15.4(\mathrm{Mol} \mathrm{3})$. The ${ }^{32} \mathrm{~S} /{ }^{34} \mathrm{~S}-$ value for the local ISM $\approx 22$ (Wilson \& Rood 1994). The difference is likely due to CS being optically thick.

Note that if the Low objects are really representatives of a very early evolutionary stage of high-mass stars, they are expected to be associated with molecular outflows. These will contribute to the broadening of the line profiles discussed above, even though the presence of multiple emission components tends to obscure the visibility of line wings in the spectra. 


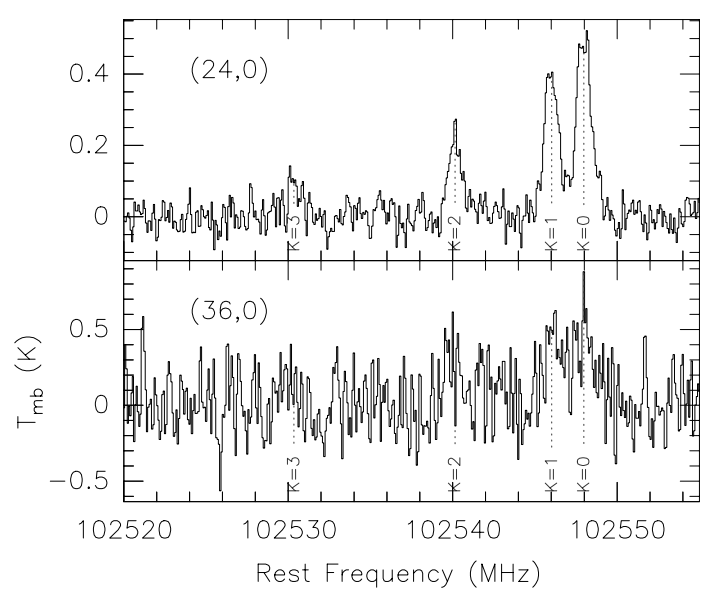

Fig. 3. c) As a), but for two positions towards Mol 98. The velocity resolution of the spectra is $0.23 \mathrm{~km} \mathrm{~s}^{-1}$; the offset position at which they were taken are indicated in the panels

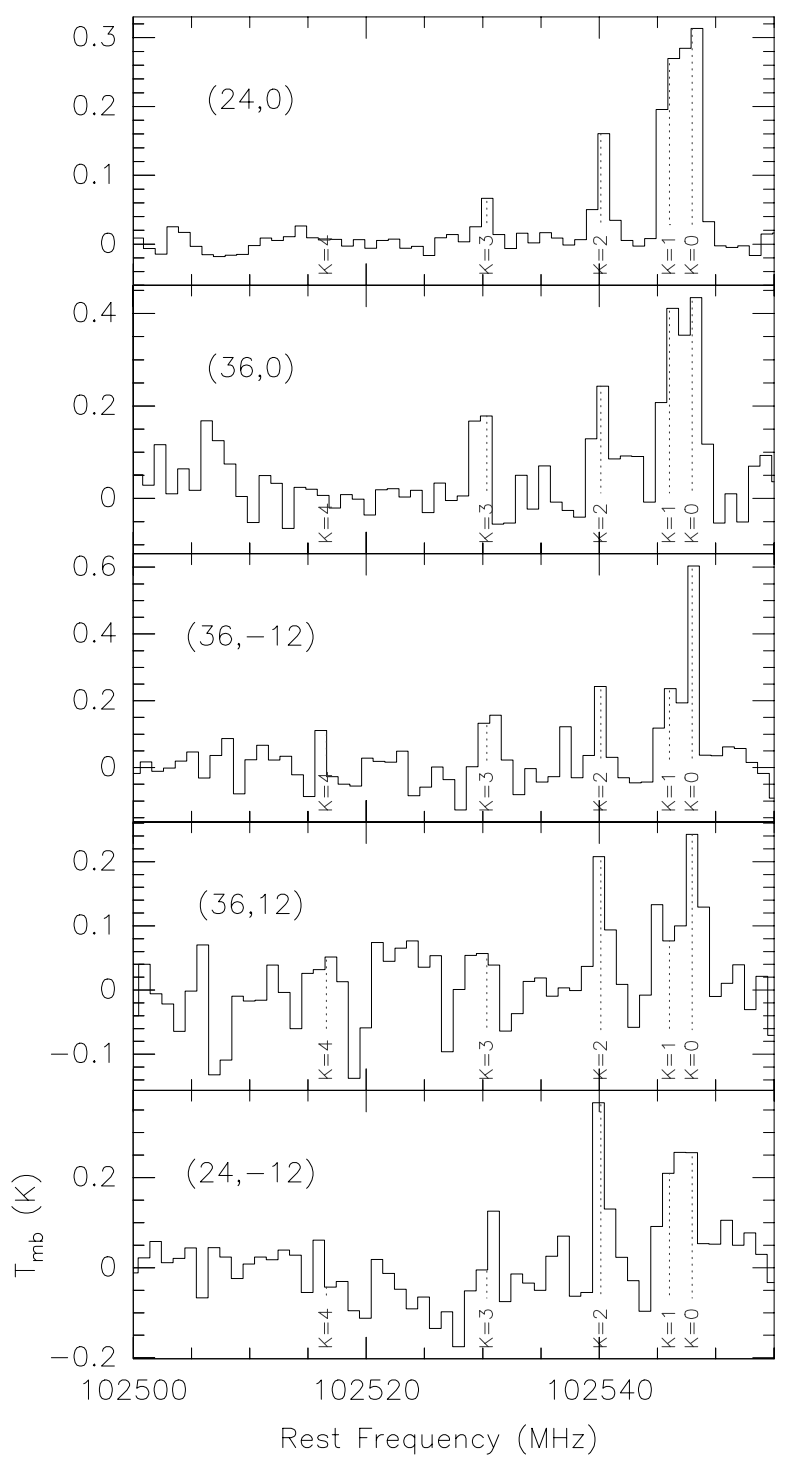

Fig. 3. d) As a), but for various positions towards Mol 98 . The velocity resolution of the spectra is $2.92 \mathrm{~km} \mathrm{~s}^{-1}$; the offset position at which they were taken are indicated in the panels

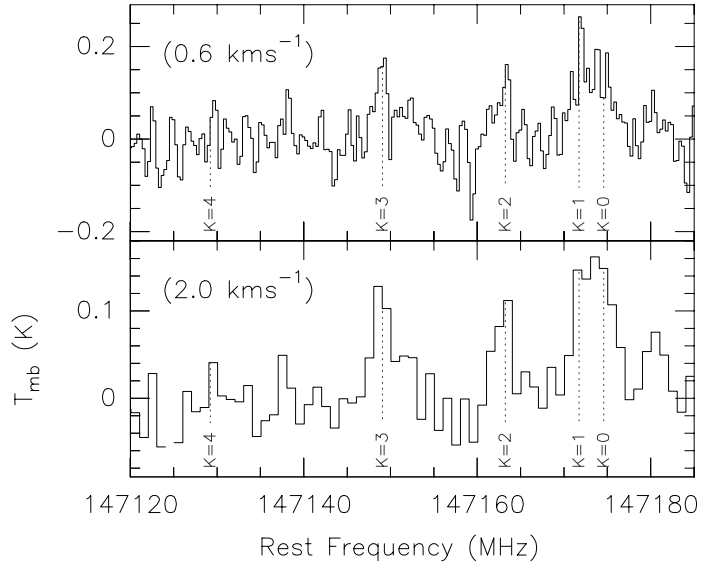

Fig. 4. Spectra of the $\mathrm{CH}_{3} \mathrm{CN}(8-7)$ rotational transition towards the peak position $\left(24^{\prime \prime}, 0^{\prime \prime}\right)$ in Mol 98 . The velocity resolution of the spectra is indicated in the panels. The location of the first few K-components are indicated in the spectra

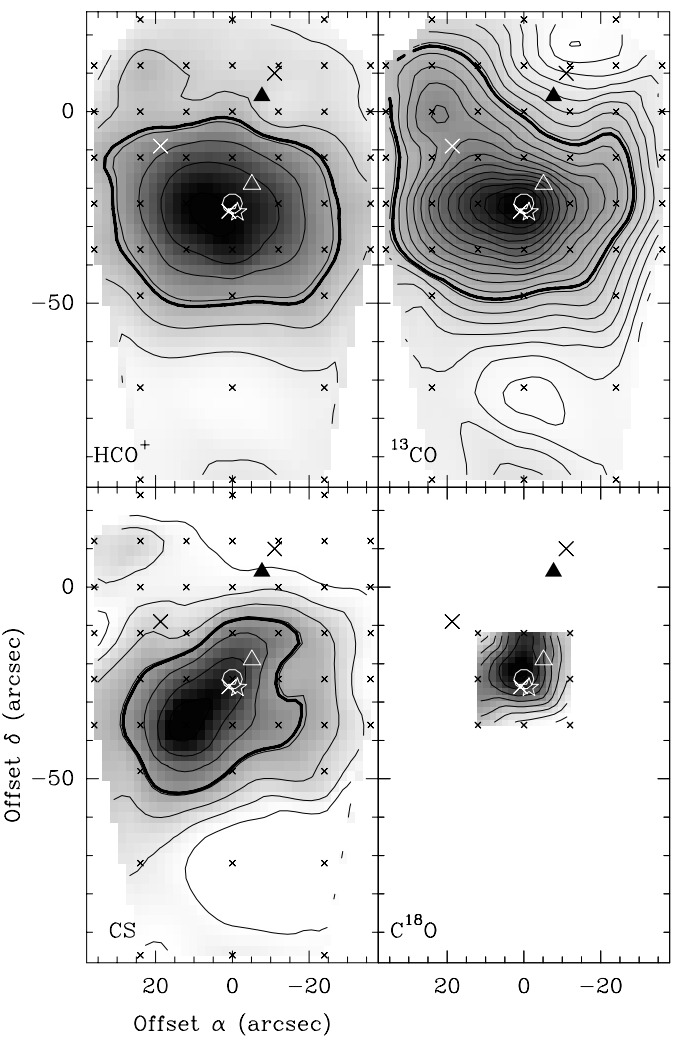

Fig. 5. Maps towards all sources of the integrated emission in the lines of $\mathrm{HCO}^{+}(1-0),{ }^{13} \mathrm{CO}(2-1), \mathrm{CS}(3-2)$, and $\mathrm{C}^{18} \mathrm{O}(2-1)$. Where $\mathrm{C}^{34} \mathrm{~S}(3-2)$ was strong enough, a panel with a map of that molecule is shown as well. Thick contours indicate the $50 \%$ level in each map. The $(0,0)$ position is the position of the sub-mm peak (Molinari et al. 2000). The small crosses mark the observed positions, the filled triangle indicates the IRAS point source position, and the circle marks the position where we made longer integrations at the frequency of the $\mathrm{CH}_{3} \mathrm{C}_{2} \mathrm{H}(6-5)$ and $\mathrm{CH}_{3} \mathrm{CN}$ lines. a) Data for Mol 3. The open triangle is the peak of the $850 \mu \mathrm{m}$ emission, while the star indicates the position of the $\mathrm{H}_{2} \mathrm{O}$ maser (Jenness et al. 1995). The big crosses indicate the positions of the peaks of the radio continuum emission (VLA D-array, $3.6 \mathrm{~cm}$; Molinari et al., in preparation). For details, see Sect. 5. Contour values (low(step)high, in $\mathrm{Kkm} \mathrm{s}^{-1}$ ) are: 0.5(1)6.5 $\left(\mathrm{HCO}^{+}\right) ; 13(3) 69$ $\left({ }^{13} \mathrm{CO}\right) ; 0.5(1) 6.5(\mathrm{CS}) ; 9(1) 17\left(\mathrm{C}^{18} \mathrm{O}\right)$ 


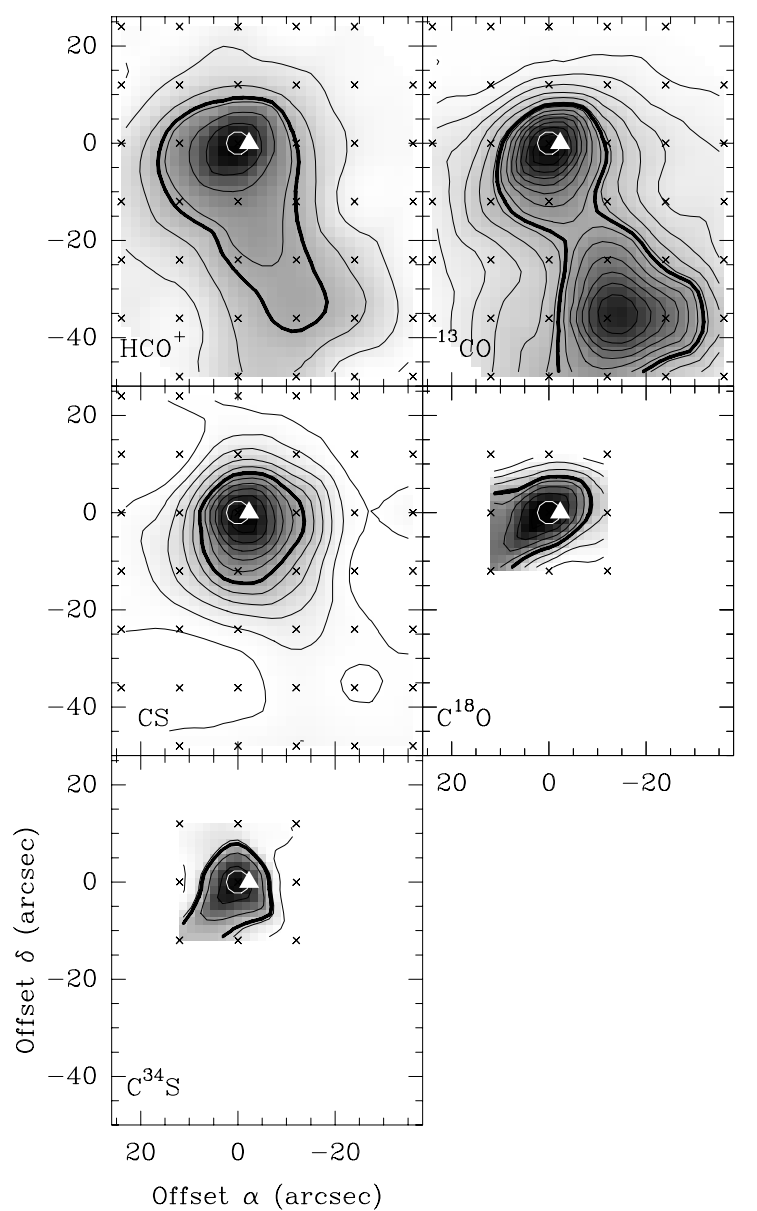

Fig. 5. b) Same as a), for Mol 8. Contour values (low (step) high, in $\left.\mathrm{Kkm} \mathrm{s}^{-1}\right)$ are: $0.5(1) 6.5\left(\mathrm{HCO}^{+}\right) ; 4.5(4) 60.5\left({ }^{13} \mathrm{CO}\right)$; $0.5(1) 11(\mathrm{CS}) ; 1(1) 8\left(\mathrm{C}^{18} \mathrm{O}\right) ; 0.4(0.4) 2\left(\mathrm{C}^{34} \mathrm{~S}\right)$

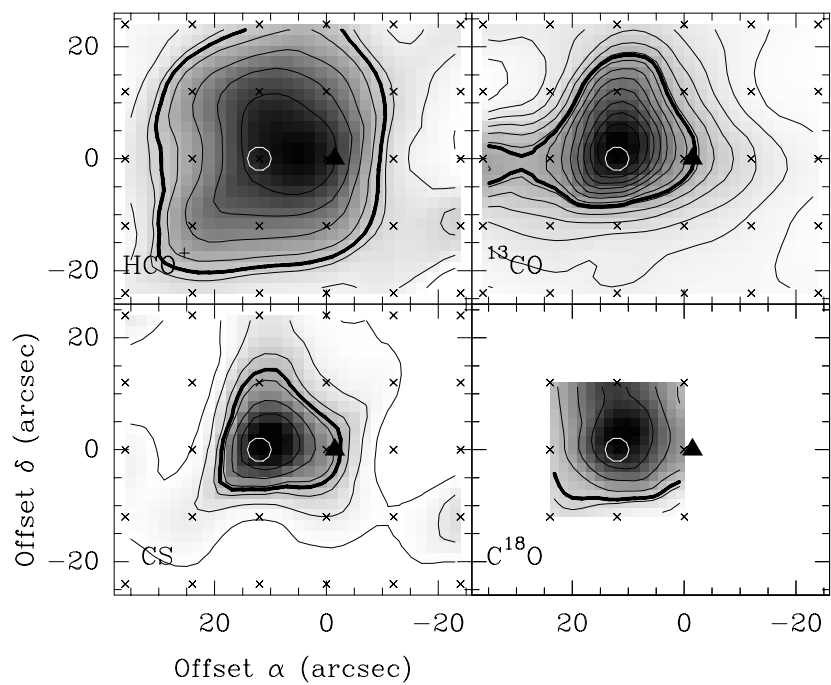

Fig. 5. c) Same as a), for Mol 59. Contour values (low (step) high, in $\left.\mathrm{Kkm} \mathrm{s}^{-1}\right)$ are: $1(0.5) 4.5\left(\mathrm{HCO}^{+}\right) ; 6(4) 62\left({ }^{13} \mathrm{CO}\right) ; 1(1) 7$ (CS); 3(2)15 ( $\left.\mathrm{C}^{18} \mathrm{O}\right)$

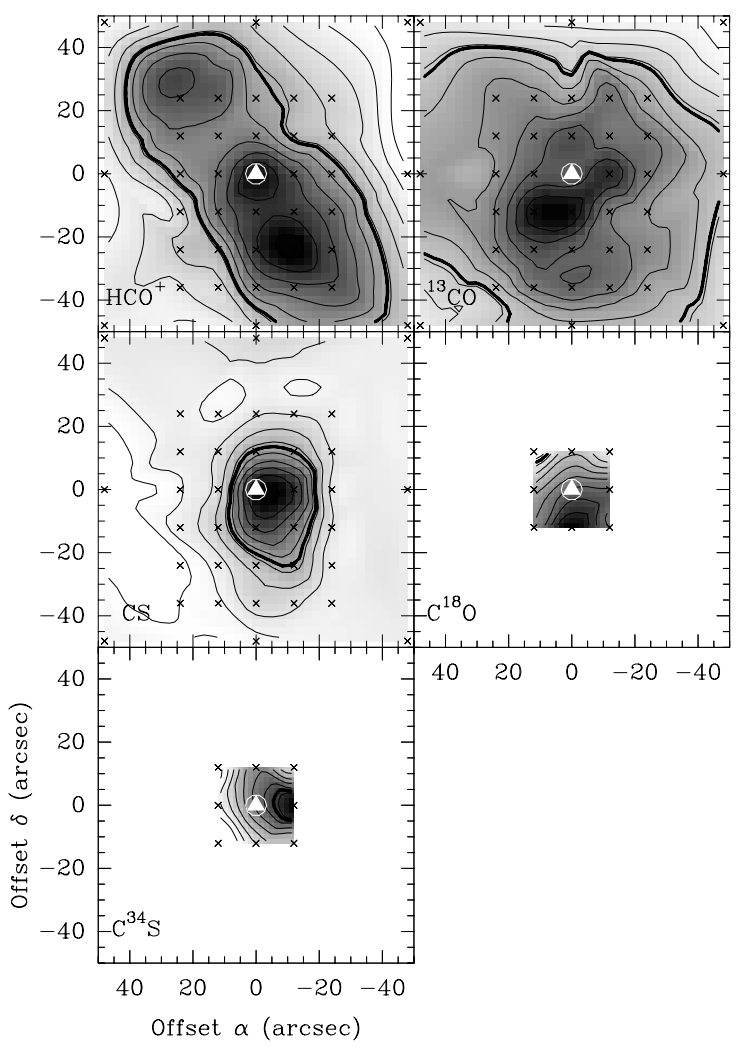

Fig. 5. d) Same as a), for Mol 75. Contour values (low (step) high, in $\mathrm{Kkm} \mathrm{s}^{-1}$ ) are: $1(1) 10\left(\mathrm{HCO}^{+}\right) ; 15(6) 81\left({ }^{13} \mathrm{CO}\right) ; 1(2) 19$ (CS); $14(2) 34\left(\mathrm{C}^{18} \mathrm{O}\right) ; 0.3(0.3) 2.7\left(\mathrm{C}^{34} \mathrm{~S}\right)$

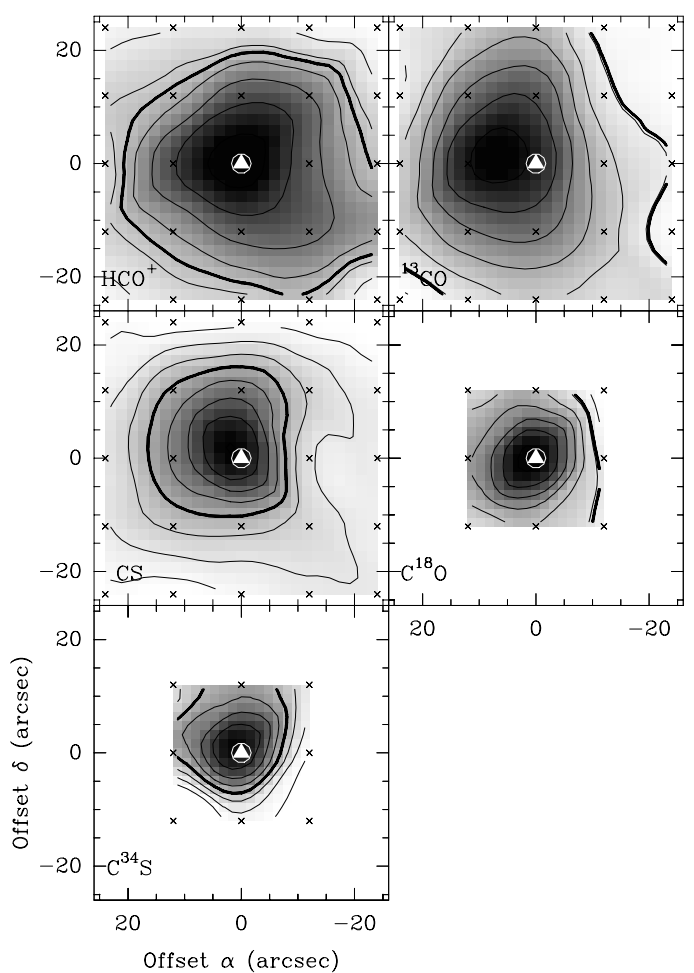

Fig. 5. e) Same as a), for Mol 77. Contour values (low (step) high, in $\left.\mathrm{Kkm} \mathrm{s}^{-1}\right)$ are: $1(0.5) 4\left(\mathrm{HCO}^{+}\right) ; 18(4) 42\left({ }^{13} \mathrm{CO}\right) ; 1(1) 8$ $(\mathrm{CS}) ; 7(2) 21\left(\mathrm{C}^{18} \mathrm{O}\right) ; 0.3(0.3) 2.4\left(\mathrm{C}^{34} \mathrm{~S}\right)$ 


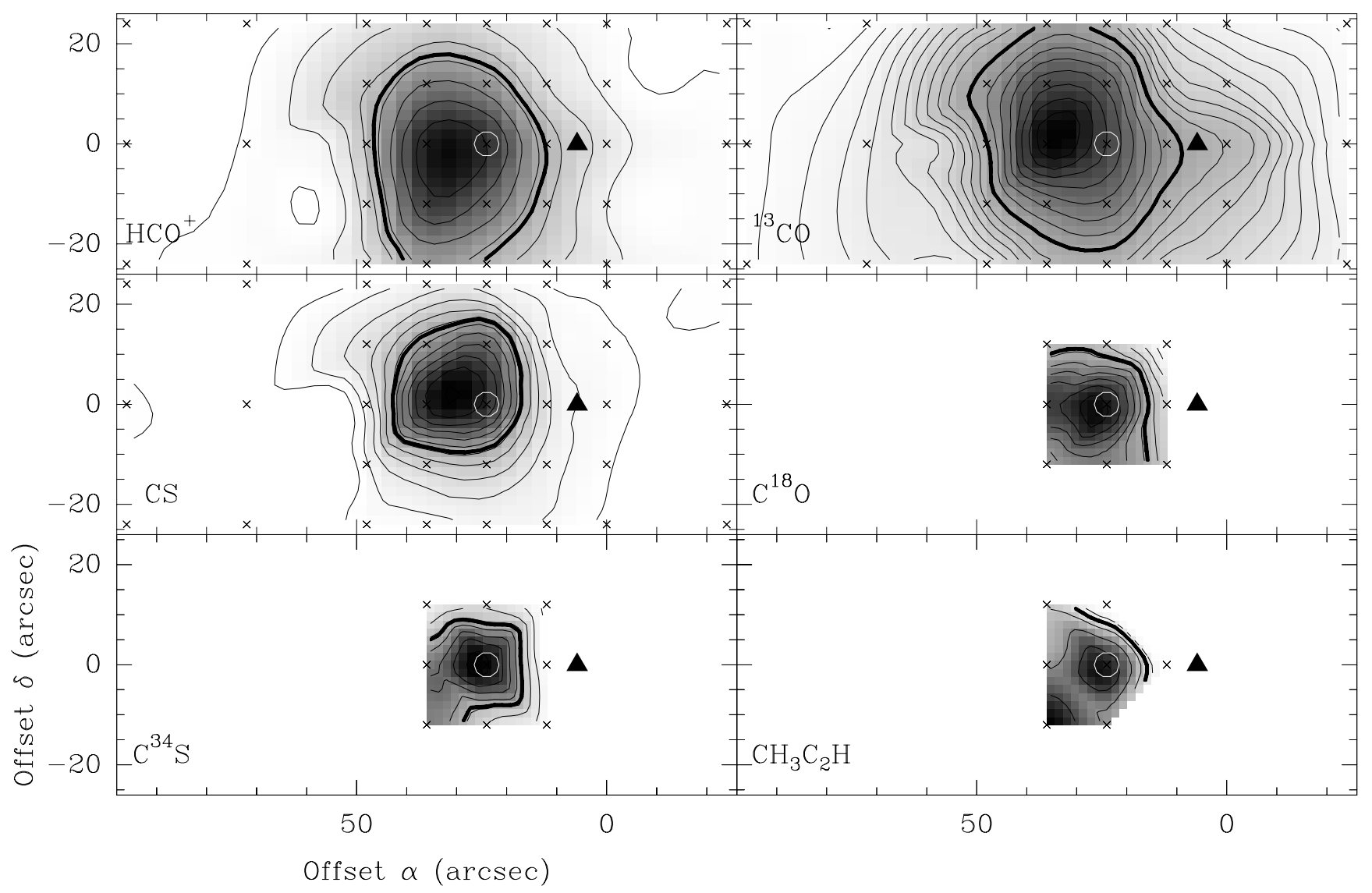

Fig. 5. f) Same as a), for Mol 98. Added here is a panel showing the distribution of the integrated emission over the $K=0,1$ components of $\mathrm{CH}_{3} \mathrm{C}_{2} \mathrm{H}(6-5)$. Contour values (low (step) high, in $\mathrm{Kkm} \mathrm{s}^{-1}$ ) are: 1(2)17 $\left(\mathrm{HCO}^{+}\right) ; 1(4) 41(6) 95\left({ }^{13} \mathrm{CO}\right) ; 1(2) 23$ (CS); $3(3) 35\left(\mathrm{C}^{18} \mathrm{O}\right) ; 0.5(0.5) 4\left(\mathrm{C}^{34} \mathrm{~S}\right) ; 1(0.5) 3.5\left(\mathrm{CH}_{3} \mathrm{C}_{2} \mathrm{H}\right)$

\subsection{Boltzmann plots}

Figure 8 shows the Boltzmann plots constructed from the $\mathrm{CH}_{3} \mathrm{C}_{2} \mathrm{H}(6-5)$ observations. From weighted least-squares fits to the data points, temperature and column densities can be derived (see e.g. Kuiper et al. 1984; Bergin et al. 1994). The resulting rotational temperature $T_{\text {rot }}$, asumed to be equal to the gas kinetic temperature $T_{\text {kin }}$, and column densities, assuming optically thin emission, are collected in Cols. 4 and 6 of Table 7 , respectively. The uncertainty in $T_{\text {kin }}$ in Col. 5 is derived from that in the slope of the fit. An uncertainty for the column density $N$ was obtained in two ways: by calculating an $N_{\text {low }}$ and $N_{\text {high }}$ with the extremes in $T_{\text {kin }}$, and taking the average difference between these two values and the $N$ derived for $T_{\text {kin }}$ (given in Col. 6); and by calculating the average difference in $N$ as a result of the uncertainty in the slope of the fit. The uncertainties in $N$ derived in these two ways were added in quadrature, and the resulting value is listed in Col. 7.

We list the parameters obtained from both the highand the low-resolution spectra, for comparison. Only for Mol 118 is there a significant difference between the two. The derived $T_{\text {kin }}$ are between 20 and $45 \mathrm{~K}$, in agreement with the dust temperatures (Molinari et al. 2000). The low temperatures for these Low sources are in marked contrast with those found for members of the High group such as IRAS20126+4104 (Mol 119), where $T_{\text {kin }} \sim 200 \mathrm{~K}$ (Cesaroni et al 1997; from $\mathrm{CH}_{3} \mathrm{CN}$ observations).

The $\mathrm{CH}_{3} \mathrm{CN}(8-7)$ data for Mol 98 at $\left(24^{\prime \prime}, 0^{\prime \prime}\right)$ yield $T_{\text {kin }} \approx 666 \mathrm{~K}(425-1535 \mathrm{~K})$ from the high-resolution spectrum, and $1073 \mathrm{~K}(577-7599 \mathrm{~K})$ from the low-resolution spectrum. Though the uncertainties are very large, it does show that the temperature derived from $\mathrm{CH}_{3} \mathrm{CN}$ is much larger than that, derived from $\mathrm{CH}_{3} \mathrm{C}_{2} \mathrm{H}$, indicating that the emission of the former originates from deeper in the cloud, i.e. from closer to the embedded heating source (i.e. the YSO).

\section{Comments on individual sources}

In the following subsections we shall discuss the observed sources in some detail. If it helps our understanding of the molecular line data, we shall make use of as yet unpublished observations of ${ }^{12} \mathrm{CO}(2-1)$ and $\mathrm{C}^{18} \mathrm{O}(2-1)$ [NRAO 12-m], 3.6-cm radio continuum [VLA D-array], and $450 \mu \mathrm{m}$ and/or $850 \mu \mathrm{m}$ [SCUBA]. We try to identify the velocity components of the molecular emission which are associated with the Molinari sources, and estimate the masses of these components from the ${ }^{13} \mathrm{CO}$ data. 


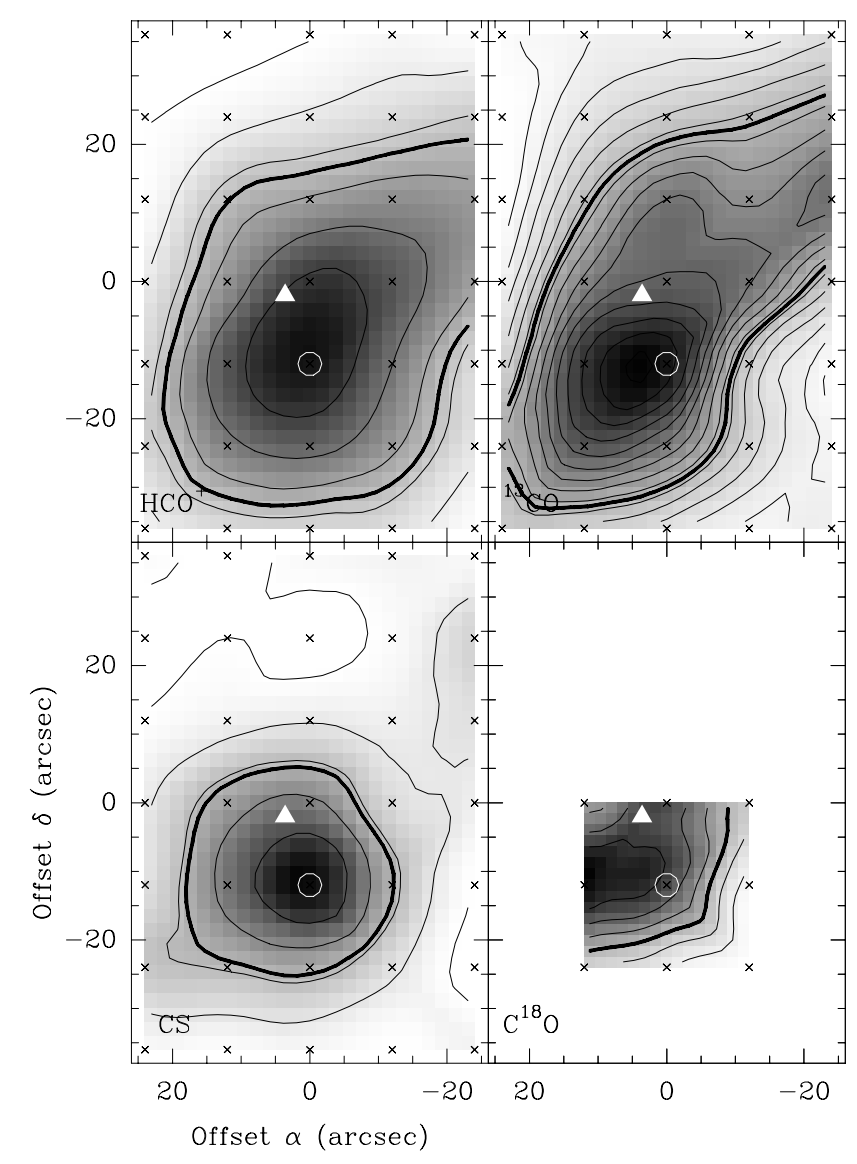

Fig. 5. g) Same as a), for Mol 117. Contour values (low(step)high, in $\mathrm{Kkm} \mathrm{s}^{-1}$ ) are: $1(1) 7\left(\mathrm{HCO}^{+}\right) ; 5(4) 70\left({ }^{13} \mathrm{CO}\right) ; 0.5(1) 5.5$ $(\mathrm{CS}) ; 1(1) 8\left(\mathrm{C}^{18} \mathrm{O}\right)$

The first step is to derive the column densities of the observed molecule, using the familiar equations (see e.g. Brand \& Wouterloot 1998; Rohlfs \& Wilson 1996) with the appropriate constants for the molecule under consideration put in, and assuming the emission is optically thin. The excitation temperature $T_{\mathrm{ex}}$ is estimated from the NRAO CO spectra. To get the column density of $\mathrm{H}_{2}$ we have used the relative abundance $\left[\mathrm{H}_{2}\right] /\left[{ }^{13} \mathrm{CO}\right]=5.010^{5}$. Masses are estimated from the average $\mathrm{H}_{2}$ column density, using all emission above the $F W H M$ level of the integrated ${ }^{13} \mathrm{CO}$ emission, and correcting the enclosed area for the beam of the observations. A correction for $\mathrm{He}(\times 1.36)$ has been applied as well. Mass estimates are lower limits, because not all emission is used, and the FWHM contour is not always closed within the area mapped with the 30-m. The results are collected in Table 8.

\subsection{Mol 3}

An optical image of a $10^{\prime} \times 10^{\prime}$ region around this object is shown in Fig. 9 (taken from the Digital Sky Survey: DSS). Clearly visible are several nebulous patches, that have been identified by Neckel \& Staude (1984), and which are known as GN0042.0-1 and GN0042.0-2 (Neckel \& Vehrenberg 1985). In Fig. 9 the easternmost cross marks the position of the sub-mm peak (Molinari et al. 2000); the IRAS position is slightly to the NW of this, and almost coincides with the star which is located at the vertex of one of the nebulous patches. Neckel \& Staude derive a photometric distance to this star (and to the star in the nebulosity to the SW) of $1.7 \mathrm{kpc}$, based on a B5 spectral type for both stars, and an assumed luminosity class V. White \& Gee (1986) observed both objects with the VLA D-array. GN0042.0-1 was not detected (at $>0.3 \mathrm{mJy} /$ beam), while GN0042.0-2 was detected at $6 \mathrm{~cm}$. The westernmost cross in Fig. 9 indicates this radio continuum peak. The source has a peak flux density of $0.9 \mathrm{mJy} /$ beam $\left(\int F \mathrm{~d} \nu=3.5 \mathrm{mJy}\right)$; the spectral type derived from the radio data is B1-2, which is a large discrepancy with the Neckel \& Staude optical data (B5). Hence, their photometric distance is uncertain, and we will use the kinematic distance.

GN0042.0-1, the collection of nebulosities nearest to Mol 3, was recently observed by Molinari et al. (in preparation) with the VLA D-array at $3.6 \mathrm{~cm}$. They detected 3 faint radio sources, one of which (peak flux density $\approx 0.19 \mathrm{mJy} /$ beam) is very close to the IRAS source. A second $3.6 \mathrm{~cm}$ source, with similar peak flux, lies at the same position as the $\mathrm{H}_{2} \mathrm{O}$ maser and very close $\left(\sim 8^{\prime \prime} \mathrm{SE}\right)$ to the $850 \mu \mathrm{m}$ peak, both measured by Jenness et al. (1995). This sub-mm peak lies $\sim-5^{\prime \prime},-19^{\prime \prime}$ from the submm peak given in Molinari et al. (2000; taken as the 0,0 


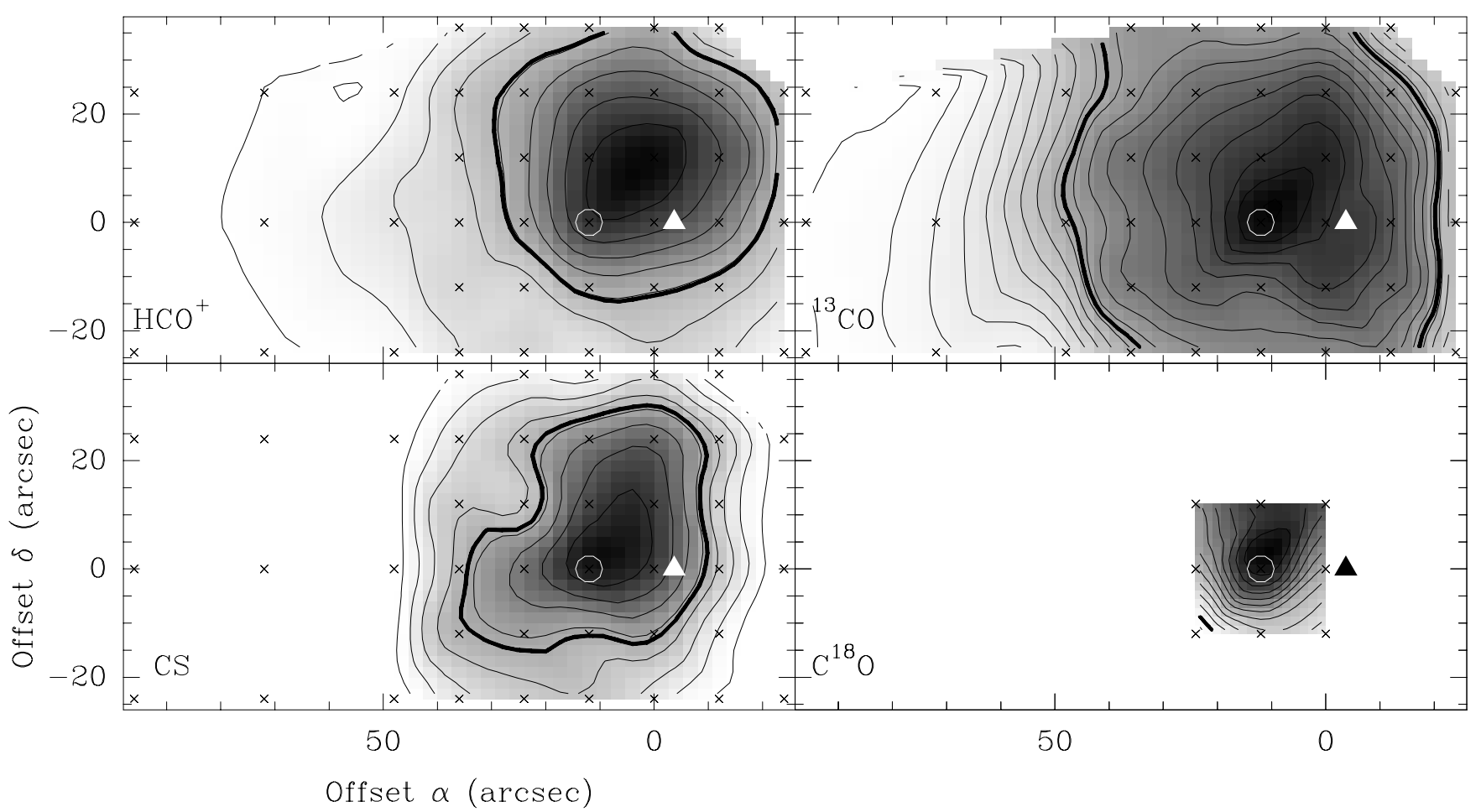

Fig. 5. h) Same as a), for Mol 118. Contour values (low (step) high, in $\mathrm{Kkm} \mathrm{s}^{-1}$ ) are: $1(1) 10\left(\mathrm{HCO}^{+}\right) ; 1(4) 71\left({ }^{13} \mathrm{CO}\right) ; 0.5(1) 8.5$

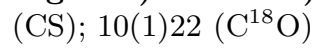

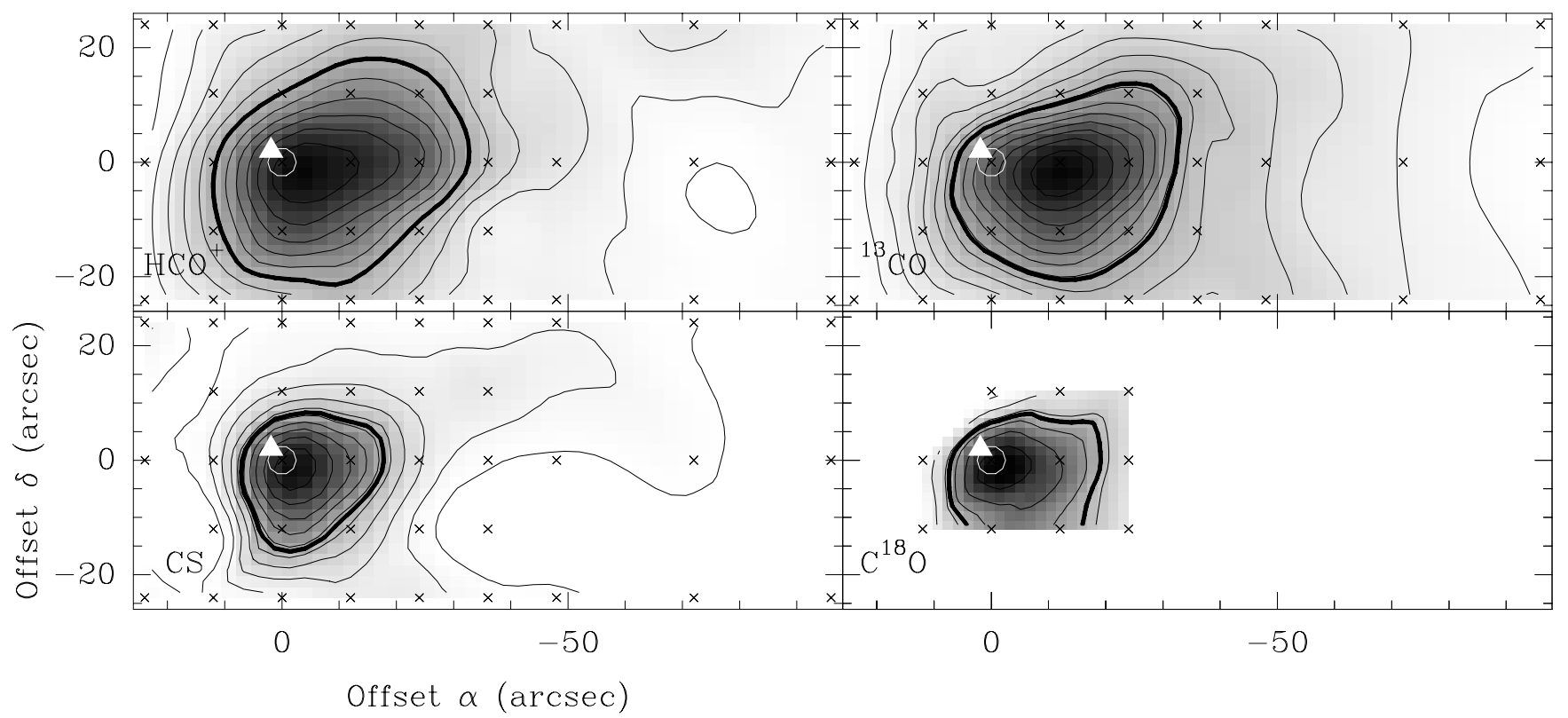

Fig. 5. i) Same as a), for Mol 136. Contour values (low (step) high, in $\mathrm{Kkm} \mathrm{s}^{-1}$ ) are: $0.5(0.5) 6\left(\mathrm{HCO}^{+}\right) ; 1(4) 57\left({ }^{13} \mathrm{CO}\right)$; $0.5(1) 10.5(\mathrm{CS}) ; 2(2) 13\left(\mathrm{C}^{18} \mathrm{O}\right)$

position of the present maps), who noted however that they missed the primary peak in their observations (a 5-point cross near the IRAS position only). Recent, unpublished SCUBA $(850 \mu \mathrm{m})$ observations by Molinari et al. show that the true sub-mm peak coincides with the Jenness et al. $850 \mu \mathrm{m}$ peak. In Fig. $5 \mathrm{a}$ it is seen that the CS peak is slightly offset to the SE with respect to the communal location of the other molecular peaks, the sub-mm peak, the $\mathrm{H}_{2} \mathrm{O}$ maser, and one of the radio continuum sources.

A look at Fig. 1a shows, that at the peak position in $\mathrm{Mol} 3$ the ${ }^{13} \mathrm{CO}, \mathrm{C}^{18} \mathrm{O}$, and $\mathrm{HCO}^{+}$spectra are double-peaked, while the CS spectrum is flat-topped. 


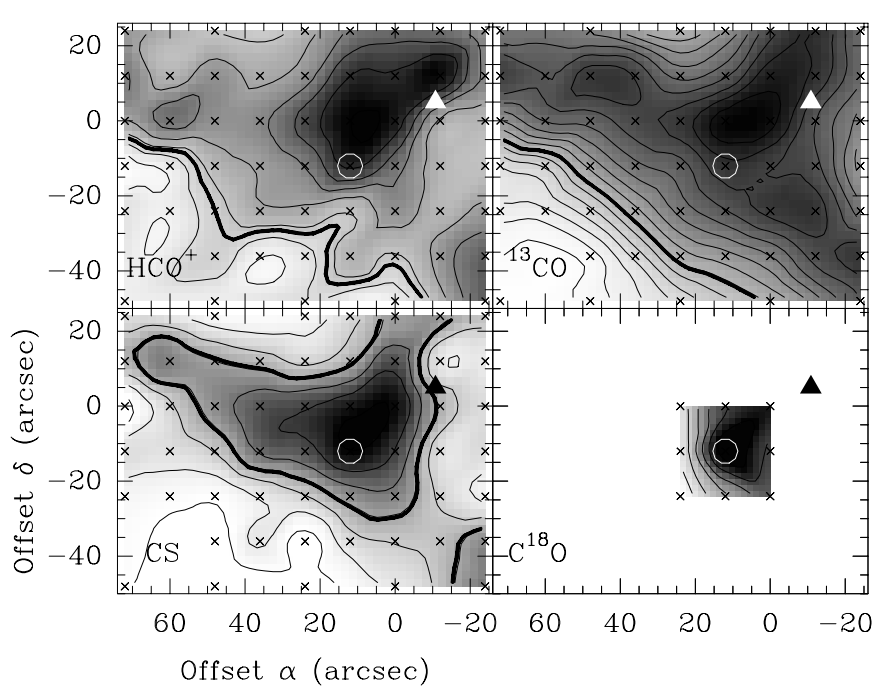

Fig. 5. j) Same as a), for Mol 155. Contour values (low (step) high, in $\left.\mathrm{Kkm} \mathrm{s}^{-1}\right)$ are: $1.5(1) 5.5\left(\mathrm{HCO}^{+}\right)$; $8(4) 72\left({ }^{13} \mathrm{CO}\right)$; $0.5(1) 6.5(\mathrm{CS}) ; 9(1) 15\left(\mathrm{C}^{18} \mathrm{O}\right)$

The velocity of the (weak) $\mathrm{C}^{34} \mathrm{~S}$ line falls more or less in between the dip in the line profiles and the peak of the red component. Inspection of all profiles in the map, and of position-velocity plots of the molecular emission shows, that two components can be clearly seen over the whole mapped region; also the CS profiles are double-peaked away from the central position. In Fig. 10 we show the integrated areas over the blue- (at $-50.85 \mathrm{~km} \mathrm{~s}^{-1}$; lefthand panels) and the red side of the dip (right-hand panels). The sub-mm peak, the $\mathrm{H}_{2} \mathrm{O}$ maser, and one of the $3.6 \mathrm{~cm}$ sources lie towards the peaks of the gas distribution for both components, although the correspondence with the blue peaks is slightly better (while the red component is more compact). In ${ }^{13} \mathrm{CO}$, blue component, a separate clump is visible, which may also be present in the $\mathrm{HCO}^{+}$and $\mathrm{CS}$ maps.

\section{2. $\mathrm{Mol} 8$}

The finding chart (at $8000 \AA$ ) published by Campbell et al. (1989) shows that some very faint diffuse emission is associated with the IRAS source, which coincides with a star-like object. This source has been included in various

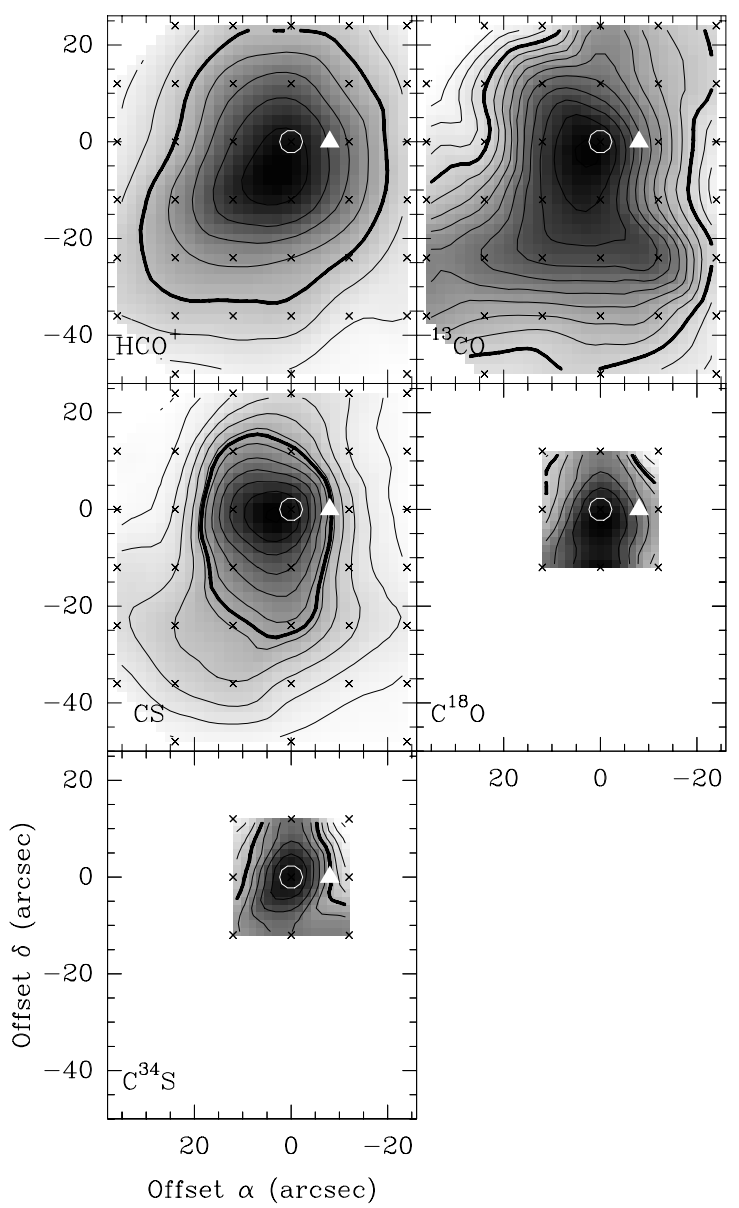

Fig. 5. k) Same as a), for Mol 160. Contour values (low (step) high, in $\mathrm{Kkm} \mathrm{s}^{-1}$ ) are: $3(2) 18\left(\mathrm{HCO}^{+}\right) ; 31(6) 110\left({ }^{13} \mathrm{CO}\right)$; $0.5(2) 22.5(\mathrm{CS}) ; 5(2) 19\left(\mathrm{C}^{18} \mathrm{O}\right) ; 0.3(0.3) 3\left(\mathrm{C}^{34} \mathrm{~S}\right)$

studies. Ishii et al. (1998) measured its NIR $(1.3-4.2 \mu \mathrm{m})$ spectrum, and found the $3.1 \mu \mathrm{m}$ absorption feature due to $\mathrm{H}_{2} \mathrm{O}$ ice. This feature is found in environments protected from UV radiation, i.e. high-density molecular clouds, and is indeed often detected in deeply embedded YSOs (Ishii et al. 1998). Slysh et al. (1997) found a strongly circularly polarized 2.7 Jy $\mathrm{OH}$ maser at $1665 \mathrm{MHz}$. No $6.7 \mathrm{GHz}$ methanol maser was detected by MacLeod et al. (1998), nor was this object detected by Harju et al. (1998) in their search for $\mathrm{SiO}$ maser emission.

Although the spectra taken at the peak position (Fig. 1b) seem to show a single emission component, the channel maps in Fig. 11 clearly show the existence of two components, at -25.5 and $-26.5 \mathrm{~km} \mathrm{~s}^{-1}$ respectively. Although the velocity difference is small, the two components have a different spatial distribution and are therefore easily separated by Gaussian fitting to the line profiles. As an illustration we show in Fig. 12 the distributions of the areas under the blue and red components for ${ }^{13} \mathrm{CO}$. The redder (northern) component is associated with the embedded object.

Mol 8 is WB89 621 in the catalogue of Wouterloot \& Brand (1989), who detected (with the IRAM 30-m) 
Table 4. Gaussfit parameters of $\mathrm{CH}_{3} \mathrm{C}_{2} \mathrm{H}(6-5)$ lines

\begin{tabular}{|c|c|c|c|c|c|c|c|c|}
\hline \multirow[b]{2}{*}{$\begin{array}{r}\text { Mol } \\
\# \\
\end{array}$} & \multirow[b]{2}{*}{$\begin{array}{c}\text { offset } \\
\left({ }^{\prime \prime}\right) \\
\end{array}$} & \multirow[b]{2}{*}{$\begin{array}{c}V_{\text {res }} \\
\left(\mathrm{km} \mathrm{s}^{-1}\right) \\
\end{array}$} & \multirow[b]{2}{*}{$\begin{array}{r}V_{\mathrm{lsr}} \\
\left(\mathrm{km} \mathrm{s}^{-1}\right) \\
\end{array}$} & \multirow[b]{2}{*}{$\begin{array}{c}\Delta v \\
\left(\mathrm{~km} \mathrm{~s}^{-1}\right)\end{array}$} & \multicolumn{4}{|c|}{$\int T_{\mathrm{mb}} \mathrm{d} v\left(\mathrm{Kkm} \mathrm{s}^{-1}\right)$} \\
\hline & & & & & $K=0$ & 1 & 2 & 3 \\
\hline \multirow[t]{2}{*}{59} & 12,0 & 0.23 & $+113.9 \pm 0.1$ & $2.98 \pm 0.09$ & $0.64 \pm 0.04$ & $0.49 \pm 0.03$ & $0.21 \pm 0.03$ & \\
\hline & & 2.92 & $+114.0 \pm 0.2$ & $4.02 \pm 0.16$ & $0.62 \pm 0.04$ & $0.48 \pm 0.04$ & $0.12 \pm 0.04$ & \\
\hline \multirow[t]{2}{*}{75} & 0,0 & 0.23 & $+57.28 \pm 0.03$ & $2.78 \pm 0.04$ & $1.31 \pm 0.03$ & $1.02 \pm 0.03$ & $0.33 \pm 0.03$ & $0.13 \pm 0.03$ \\
\hline & & 2.92 & $+57.18 \pm 0.11$ & $3.62 \pm 0.24$ & $1.25 \pm 0.04$ & $1.03 \pm 0.04$ & $0.33 \pm 0.04$ & $0.12 \pm 0.04$ \\
\hline \multirow[t]{2}{*}{77} & 0,0 & 0.23 & $+76.04 \pm 0.04$ & $1.52 \pm 0.05$ & $0.50 \pm 0.03$ & $0.46 \pm 0.03$ & $0.10 \pm 0.03$ & $0.06 \pm 0.03$ \\
\hline & & 2.92 & $+76.04 \pm 0.05$ & $2.36 \pm 0.58$ & $0.50 \pm 0.23$ & $0.57 \pm 0.27$ & $0.13 \pm 0.06$ & $0.04 \pm 0.03$ \\
\hline \multirow[t]{7}{*}{98} & 24,0 & 0.23 & $+57.79 \pm 0.03$ & $3.06 \pm 0.04$ & $1.68 \pm 0.04$ & $1.32 \pm 0.04$ & $0.69 \pm 0.04$ & $0.29 \pm 0.04$ \\
\hline & & 2.92 & $+57.68 \pm 0.07$ & $4.33 \pm 0.14$ & $1.80 \pm 0.05$ & $1.43 \pm 0.06$ & $0.74 \pm 0.05$ & $0.31 \pm 0.05$ \\
\hline & 36,0 & 0.23 & $+57.48 \pm 0.21$ & $3.89 \pm 0.23$ & $2.11 \pm 0.26$ & $1.92 \pm 0.25$ & $1.23 \pm 0.25$ & $0.56 \pm 0.24$ \\
\hline & & 2.92 & $+57.43 \pm 0.21$ & $4.75 \pm 0.26$ & $2.43 \pm 0.21$ & $2.02 \pm 0.21$ & $1.30 \pm 0.21$ & $0.88 \pm 0.20$ \\
\hline & $36,-12$ & 2.92 & $+56.83 \pm 0.41$ & $3.77 \pm 0.26$ & $2.45 \pm 0.35$ & $1.00 \pm 0.31$ & $0.95 \pm 0.31$ & $0.72 \pm 0.34$ \\
\hline & 36,12 & 2.92 & $+56.43 \pm 0.49$ & $4.96 \pm 0.48$ & $1.28 \pm 0.27$ & $0.50 \pm 0.25$ & $1.07 \pm 0.26$ & $0.34 \pm 0.25$ \\
\hline & $24,-12$ & 2.92 & $+56.32 \pm 0.61$ & $4.45 \pm 0.47$ & $1.25 \pm 0.43$ & $1.20 \pm 0.43$ & $1.64 \pm 0.40$ & \\
\hline \multirow[t]{2}{*}{118} & 12,0 & 0.23 & $+7.92 \pm 0.04$ & $1.55 \pm 0.06$ & $0.41 \pm 0.03$ & $0.29 \pm 0.02$ & $0.05 \pm 0.02$ & \\
\hline & & 2.92 & $+8.16 \pm 0.28$ & $3.96 \pm 0.56$ & $0.39 \pm 0.05$ & $0.21 \pm 0.04$ & $0.07 \pm 0.04$ & $0.07 \pm 0.04$ \\
\hline \multirow[t]{2}{*}{160} & 0,0 & 0.23 & $-50.45 \pm 0.06$ & $1.85 \pm 0.08$ & $0.28 \pm 0.03$ & $0.31 \pm 0.03$ & $0.14 \pm 0.03$ & $0.05 \pm 0.03$ \\
\hline & & 2.92 & $-50.48 \pm 0.25$ & $4.17 \pm 0.41$ & $0.41 \pm 0.05$ & $0.47 \pm 0.05$ & $0.19 \pm 0.05$ & $0.07 \pm 0.05$ \\
\hline
\end{tabular}

Table 5. Gaussfit parameters of $\mathrm{CH}_{3} \mathrm{CN}(8-7)$ lines

\begin{tabular}{|c|c|c|c|c|c|c|c|c|}
\hline \multirow[b]{2}{*}{$\begin{array}{r}\text { Mol } \\
\#\end{array}$} & \multirow[b]{2}{*}{$\begin{array}{c}\text { offset } \\
\left({ }^{\prime \prime}\right)\end{array}$} & \multirow[b]{2}{*}{$\begin{array}{c}V_{\text {res }} \\
\left(\mathrm{km} \mathrm{s}^{-1}\right)\end{array}$} & \multirow[b]{2}{*}{$\begin{array}{r}V_{\mathrm{lsr}} \\
\left(\mathrm{km} \mathrm{s}^{-1}\right)\end{array}$} & \multirow[b]{2}{*}{$\begin{array}{c}\Delta v \\
\left(\mathrm{~km} \mathrm{~s}^{-1}\right)\end{array}$} & \multicolumn{4}{|c|}{$\int T_{\mathrm{mb}} \mathrm{d} v\left(\mathrm{Kkm} \mathrm{s}^{-1}\right)$} \\
\hline & & & & & $K=0$ & 1 & 2 & 3 \\
\hline 98 & 24,0 & 0.64 & $+58.31 \pm 0.28$ & $4.85 \pm 0.36$ & $0.77 \pm 0.11$ & $0.88 \pm 0.12$ & $0.51 \pm 0.11$ & $0.67 \pm 0.11$ \\
\hline & & 2.04 & $+58.12 \pm 0.40$ & $5.65 \pm 0.51$ & $0.93 \pm 0.15$ & $0.77 \pm 0.15$ & $0.59 \pm 0.14$ & $0.74 \pm 0.14$ \\
\hline
\end{tabular}

a strong $\mathrm{CO}(1-0)$ line at the IRAS position, and found evidence for wings. Wings are also visible in the present $\left({ }^{13} \mathrm{CO}, \mathrm{HCO}^{+}, \mathrm{CS}\right)$ spectra. Position-velocity plots show that wing emission occurs primarily in an area within $12^{\prime \prime}$ from map center. The blue and red lobes are centered on the IRAS/sub-mm peak position; the closeness of the peaks of the lobes suggests that the bipolar flow is seen nearly pole-on.

\subsection{Mol 59}

The distance $(5.7 \mathrm{kpc})$ to this object was based on $V_{\text {lsr }}\left[\mathrm{NH}_{3}(1,1)\right]=93.7 \mathrm{~km} \mathrm{~s}^{-1}$. This line is however very weak $\left(T_{\mathrm{mb}}=0.34 \pm 0.09 \mathrm{~K}\right)$, and the $(2,2)$ line $(0.23 \pm$ $0.10 \mathrm{~K}$ ) is found at $V_{\mathrm{lsr}}=97.7 \mathrm{~km} \mathrm{~s}^{-1}$ (Molinari et al. 1996), casting some doubt on the validity of this distance calculation. The lines measured with the $30-\mathrm{m}$ are however detected with reasonable signal-to-noise, and the emission is moreover clearly associated with Mol 59, as can be seen from Fig. 5 c. The average velocity of $\approx 114.5 \mathrm{~km} \mathrm{~s}^{-1}$, corresponds to $d_{\text {kin }}=6.6 \mathrm{kpc}$ (using the Brand \& Blitz 1993, rotation curve).
This object lies in the galactic plane, $\sim 20^{\circ}$ from the Galactic Center, and there is emission at various velocities. In the ${ }^{13} \mathrm{CO}$ spectra we also found emission at $67 \mathrm{~km} \mathrm{~s}^{-1}$ $(\sim 8 \mathrm{~K})$ for instance. In the NRAO ${ }^{12} \mathrm{CO}$ spectra, which have a larger velocity range, we find emission at practically all velocities between 0 and $170 \mathrm{~km} \mathrm{~s}^{-1}$. In those spectra, emission is also found at $\sim 117-128 \mathrm{~km} \mathrm{~s}^{-1}$; in the ${ }^{13} \mathrm{CO}$ spectra (e.g. Fig. 1c) this is visible too, sometimes as a red shoulder to the line which has its peak at $\sim 115 \mathrm{~km} \mathrm{~s}^{-1}$.

The spectra in Fig. 1c show a depression at $114.3 \mathrm{~km} \mathrm{~s}^{-1}$, at least in $\mathrm{HCO}^{+},{ }^{13} \mathrm{CO}$, and perhaps even in $\mathrm{CS}$. The $\mathrm{C}^{34} \mathrm{~S}$ line, although weak, peaks more or less at the velocity of this depression, as the $\mathrm{CH}_{3} \mathrm{C}_{2} \mathrm{H}$ and $\mathrm{C}^{18} \mathrm{O}$ lines seem to do. All $\mathrm{HCO}^{+}$and most ${ }^{13} \mathrm{CO}$ spectra in the mapped region (30 spectra, covering an area of $\left.60^{\prime \prime} \times 48^{\prime \prime}\right)$ show this dip (the ${ }^{13} \mathrm{CO}$ spectra at the edge of the map show asymmetries or shoulders), whereas CS has been detected at only a few positions near the peak at $\left(12^{\prime \prime}, 0^{\prime \prime}\right)$. This large area, and the relatively large beams involved in the observations, make self-absorption due to infalling gas an unlikely interpretation, also because there is little consistency between the relative strengths of the 
Table 6. Deconvolved source equivalent diameter (arcsec, pc) in different lines, and mass ${ }^{1}$

\begin{tabular}{|c|c|c|c|c|c|c|}
\hline $\begin{array}{r}\text { Mol } \\
\# \\
\end{array}$ & $\begin{array}{r}d \\
(\mathrm{kpc}) \\
\end{array}$ & $\begin{array}{r}\mathrm{HCO}^{+}(1-0) \\
\left({ }^{\prime \prime}, \mathrm{pc}\right) \\
\end{array}$ & $\begin{array}{r}{ }^{13} \mathrm{CO}(2-1) \\
\left({ }^{\prime \prime}, \mathrm{pc}\right) \\
\end{array}$ & $\begin{array}{r}\mathrm{CS}(3-2) \\
\left({ }^{\prime \prime}, \mathrm{pc}\right) \\
\end{array}$ & $\begin{array}{r}\mathrm{C}^{18} \mathrm{O}(2-1)^{2} \\
\left({ }^{\prime \prime}, \mathrm{pc}\right) \\
\end{array}$ & $\begin{array}{l}\operatorname{Mass}^{1} \\
\left(10^{3} M_{\odot}\right)\end{array}$ \\
\hline 3 & 7.7 & $46,1.7$ & $\gtrsim 59, \gtrsim 2.2$ & $39,1.5$ & n.d. & 2.8 \\
\hline 8 & 10.8 & $23,1.2$ & $\gtrsim 37, \gtrsim 1.9$ & $17,0.9$ & n.d. & 1.6 \\
\hline 59 & 6.6 & $\gtrsim 35, \gtrsim 1.1$ & $>25,>0.8$ & $13,0.4$ & n.d. & 0.25 \\
\hline 75 & 3.9 & $\gtrsim 70, \gtrsim 1.3$ & $>93,>1.8$ & $28,0.5$ & n.d. & 1.3 \\
\hline 77 & 5.3 & $\underset{\sim}{\sim} 34, \underset{\sim}{\sim} 0.9$ & $>52,>1.3$ & $21,0.5$ & n.d. & 0.34 \\
\hline 98 & 4.5 & $\gtrsim 27, \gtrsim 0.6$ & $39,0.9$ & $21,0.5$ & n.d. & 0.42 \\
\hline 117 & 8.7 & $\gtrsim 41, \gtrsim 1.7$ & $\gtrsim 46, \gtrsim 1.9$ & $25,1.1$ & n.d. & 1.7 \\
\hline 118 & 1.6 & $\sim 44, \sim 0.4$ & $>66,>0.5$ & $40,0.3$ & n.d. & 0.13 \\
\hline 136 & 6.2 & $30,0.9$ & $34,1.0$ & $17,0.5$ & $>21,>0.6$ & 0.37 \\
\hline 155 & 5.2 & $>81,>2.0$ & $>84,>2.1$ & $\gtrsim 51, \gtrsim 1.3$ & n.d. & 2.2 \\
\hline 160 & 4.9 & $46,1.1$ & $>65,>1.5$ & $30,0.7$ & n.d. & 2.0 \\
\hline
\end{tabular}

blue and red peaks in the profiles of the different tracers. The dip could be due to only a temperature gradient (without infall) in the cloud, or to an intervening colder cloud at the same velocity, but we prefer to interpret the spectra as being due to the superposition of several components, although this does not exclude the presence of self-absorption at the peak position. We integrated the emission over 5 velocity intervals, distinguishing between 5 components: 101-105 (comp. 1), 105-111 (comp. 2), 111-114.3 (comp. 3), 114.3-117 (comp. 4), and 117-122 (comp. 5) $\mathrm{km} \mathrm{s}^{-1}$. The first component is present mostly in the $\mathrm{E}$ part of the map, and appears to have its maximum outside the mapped region. The distribution of the integrated ${ }^{13} \mathrm{CO}$ emission from components (2) to (5) is shown in Fig. 13, together with that of components (3) and (4) for $\mathrm{HCO}^{+}$(where the other components are much fainter or absent). Components (3) and (4) are dominating the spectra, and are referred to as the "main line blue" and "main line red", respectively. Component (2) may be outflow emission connected to component (3), while (5) is most likely not outflowing gas, but emission from (a) separate component(s) (based on inspection of the 12-m ${ }^{12} \mathrm{CO}$ spectrum). From Fig. 1c it is not evident which of the two main lines is associated with the sub-mm peak, but Fig. 13 suggests that it is the red component, which is more compact than the distribution of the blue line, and which has a maximum at the sub-mm peak in both ${ }^{13} \mathrm{CO}$ and $\mathrm{HCO}^{+}$.

\section{4. $\mathrm{Mol} 75$}

Ishii et al. (1998) measured the NIR $(1.3-4.2 \mu \mathrm{m})$ spectrum of this source, and found the $3.1 \mu \mathrm{m}$ absorption feature due to $\mathrm{H}_{2} \mathrm{O}$ ice. As for $\mathrm{Mol} 3$, this is an indication of the presence of high-density material associated with this object. No $6.7 \mathrm{GHz}$ methanol maser was detected by MacLeod et al. (1998).
Table 7. Temperature and column densities, derived from the Boltzmann plots of $\mathrm{CH}_{3} \mathrm{C}_{2} \mathrm{H}(6-5)$ (see Fig. 8)

\begin{tabular}{rcccccc}
\hline $\begin{array}{r}\text { Mol } \\
\#\end{array}$ & $\begin{array}{c}\Delta \alpha, \Delta \delta \\
\left({ }^{\prime \prime},{ }^{\prime \prime}\right)\end{array}$ & $\begin{array}{c}\mathrm{km} \mathrm{s}^{1} \\
\mathrm{~km} \mathrm{~s}^{-1}\end{array}$ & $\begin{array}{c}T_{\mathrm{k}} \\
\mathrm{K}\end{array}$ & $\begin{array}{c}\delta T_{\mathrm{k}} \\
\mathrm{K}\end{array}$ & $\begin{array}{c}N \\
10^{14} \mathrm{~cm}^{-2}\end{array}$ & $\begin{array}{c}\delta N \\
10^{13} \mathrm{~cm}^{-2}\end{array}$ \\
\hline 59 & 12,0 & 0.23 & 28.6 & 0.0 & 0.35 & 0.00 \\
& & 2.92 & 21.8 & 1.2 & 0.27 & 0.25 \\
75 & 0,0 & 0.23 & 27.3 & 0.5 & 0.69 & 0.21 \\
& & 2.92 & 27.7 & 0.6 & 0.69 & 0.25 \\
77 & 0,0 & 0.23 & 32.1 & 3.0 & 0.33 & 0.51 \\
& & 2.92 & 24.0 & 3.4 & 0.27 & 0.76 \\
98 & 24,0 & 0.23 & 40.4 & 0.3 & 1.35 & 0.14 \\
& & 2.92 & 40.0 & 0.4 & 1.42 & 0.20 \\
118 & \multirow{2}{*}{12,0} & 0.23 & 18.4 & 2.0 & 0.15 & 0.28 \\
& & 2.92 & 45.9 & 5.3 & 0.45 & 0.81 \\
160 & 0,0 & 0.23 & 48.4 & 3.6 & 0.33 & 0.39 \\
& & 2.92 & 45.9 & 5.3 & 0.45 & 0.81 \\
\hline
\end{tabular}

${ }^{1}$ Resolution of the spectrum.

Our observations show that the gas around the IRAS source is distributed over several clumps. This is particularly so in ${ }^{13} \mathrm{CO}$, where lines are found at $V_{\mathrm{lsr}} \approx$ $41,49,53,56-58$ and $58-60 \mathrm{~km} \mathrm{~s}^{-1}$. The latter two components are also found in $\mathrm{HCO}^{+}$and $\mathrm{CS}$, suggesting that one or both of these are the associated components. We note that the $\mathrm{NH}_{3}(1,1)$ line is at $56.8 \mathrm{~km} \mathrm{~s}^{-1}$, and that at the map's peak position all lines (including $\mathrm{CH}_{3} \mathrm{C}_{2} \mathrm{H}$ ) detected with the $30-\mathrm{m}$, except $\mathrm{HCO}^{+}$, have $V_{\mathrm{lsr}} \approx 57 \mathrm{kms}^{-1}$. The $\mathrm{HCO}^{+}$line has a dominant component at $\sim 60 \mathrm{~km} \mathrm{~s}^{-1}$, and the dip seen in Fig. 1d is at or close to the velocity where the other lines have their peak (except for CS, which peaks at the velocity of the blue component of the $\mathrm{HCO}^{+}$emission). Note also that the $\mathrm{KOSMA} \mathrm{HCO}^{+}(4-3)$ spectrum seems to peak at the dip in the (1-0) spectrum (Fig. 2a), but Fig. 14 suggests the presence of two components. 


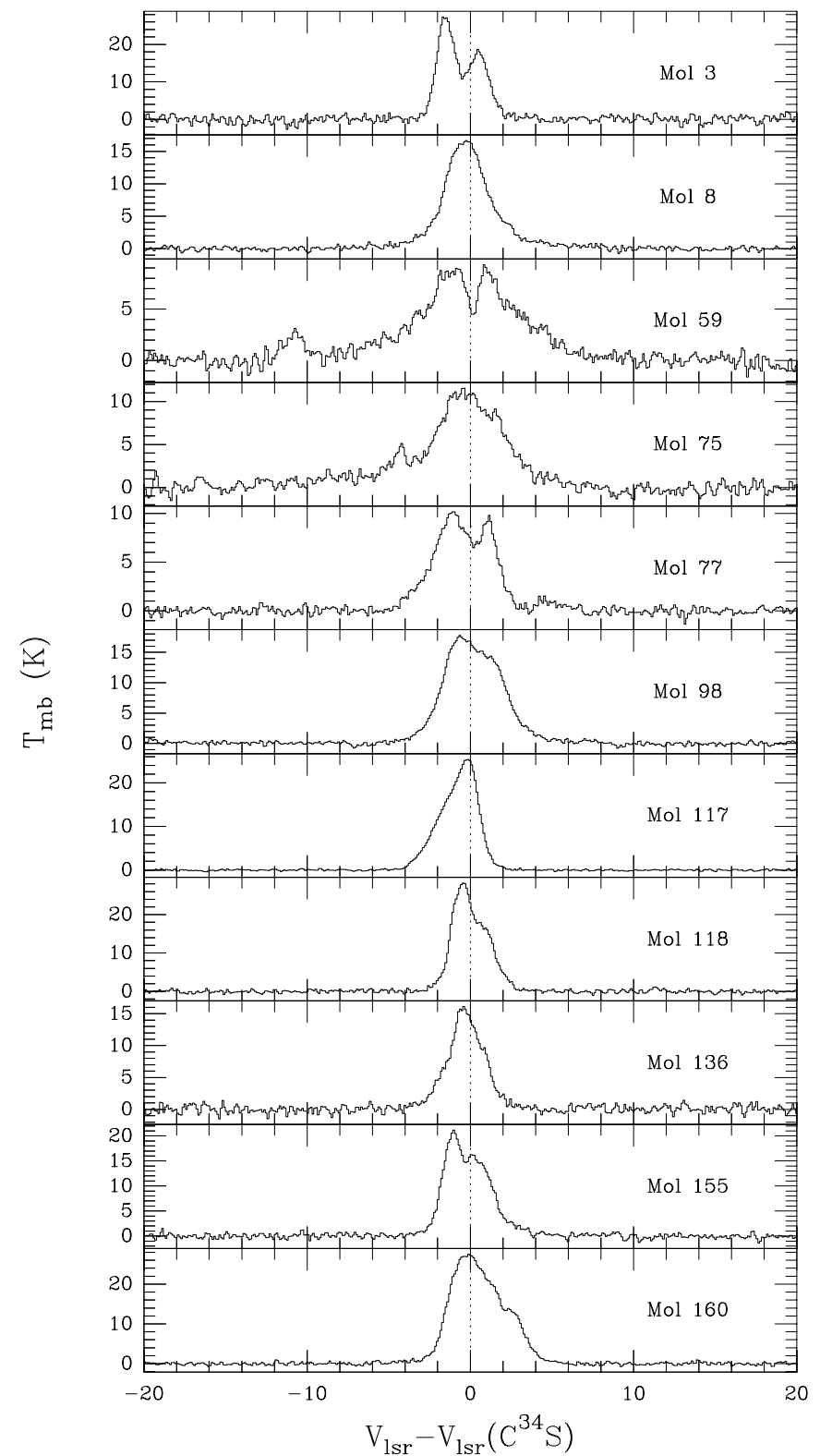

Fig. 6. a) Spectra of the ${ }^{13} \mathrm{CO}(2-1)$ emission towards the peak position in each source (as indicated in Fig. 1). The velocity is indicated with respect to that of the $\mathrm{C}^{34} \mathrm{~S}(3-2)$ line, taken to respresent the velocity of the bulk of the high-density gas

We separated the two main components by integrating over the appropriate velocity intervals $(55-58.6-$ $61.5,55-57.6-65$, and $54-58.2-62$ for ${ }^{13} \mathrm{CO}, \mathrm{HCO}^{+}$, and CS, respectively). The resulting distributions of the integrated emission are shown in Fig. 14. From this figure it is seen that the blue component peaks at or near the position of the IRAS source $/ 850 \mu \mathrm{m}$ peak, while the red component peaks south of that. Because the $\mathrm{NH}_{3}$ and $\mathrm{CH}_{3} \mathrm{C}_{2} \mathrm{H}$ lines have the same velocity as the blue component, we assume that this defines the clump in which the FIR source is embedded.

We note that the $\mathrm{HCO}^{+}$spectra in the central region of the map show a very broad red wing, up to $V_{\text {lsr }} \sim 80 \mathrm{~km} \mathrm{~s}^{-1}$ at $\left(0^{\prime \prime}, 0^{\prime \prime}\right)$ (i.e. more than $20 \mathrm{~km} \mathrm{~s}^{-1}$

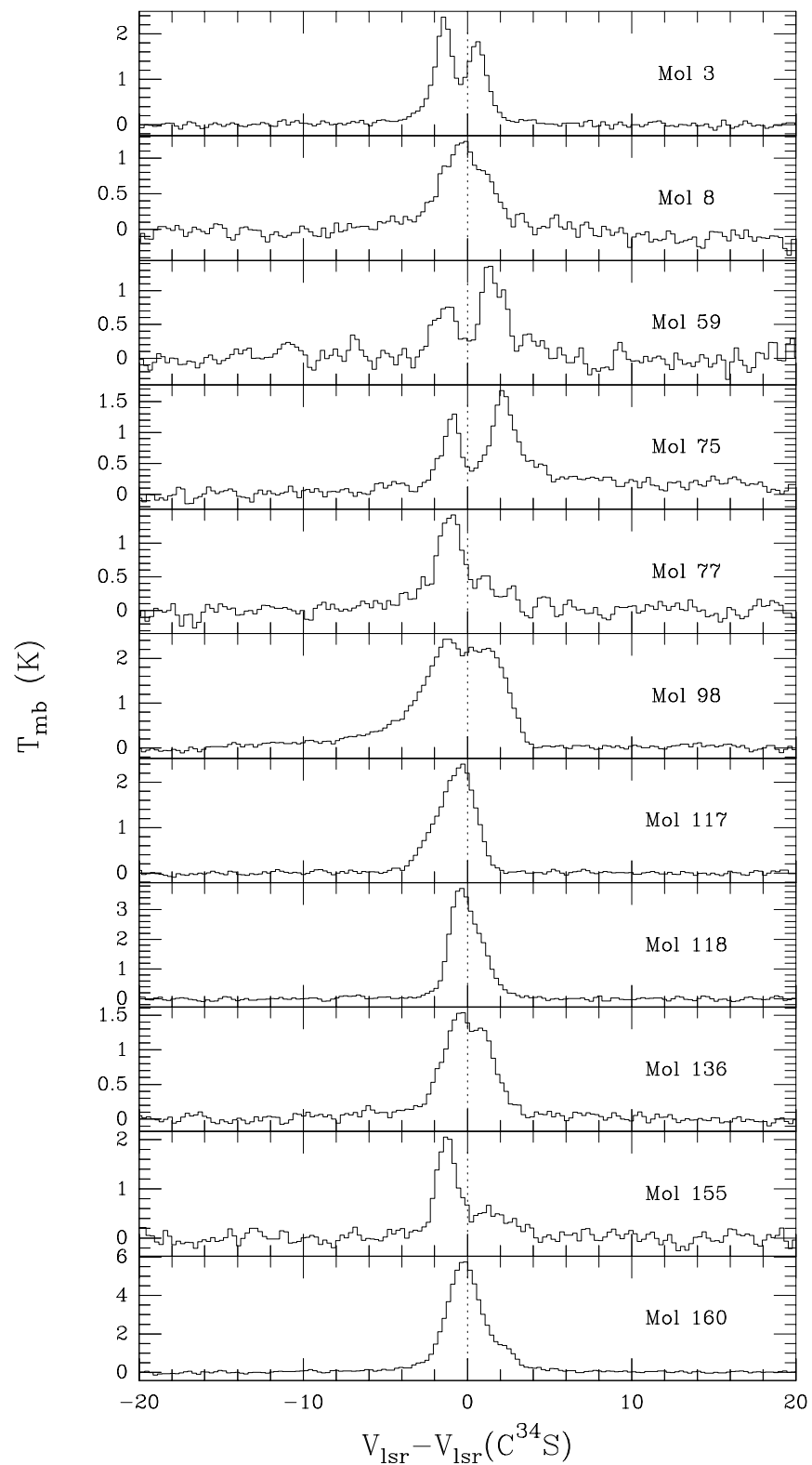

Fig. 6. b) As a), but for the $\mathrm{HCO}^{+}(1-0)$ spectra

from the center of the red component; Fig. 1d). This broad emission is found in all the individual observations (ranging in number from 2 to 8 ) contributing to the average spectrum, and is therefore unlikely to be an artifact. This is strengthened by the fact that the wing is also present in the $\mathrm{HCO}^{+}(4-3)$ spectrum (see Fig. 2). Some extended emision on the red side of the ${ }^{13} \mathrm{CO}$ spectra is also present, although with a much smaller extent in velocity (up to $6 \mathrm{~km} \mathrm{~s}^{-1}$ from the line center).

\section{5. $\mathrm{Mol} 77$}

The ${ }^{13} \mathrm{CO}$ spectrum at $\left(0^{\prime \prime}, 0^{\prime \prime}\right)$ is double-peaked (Fig. 1e). All spectra of this molecule show either two peaks, or a 


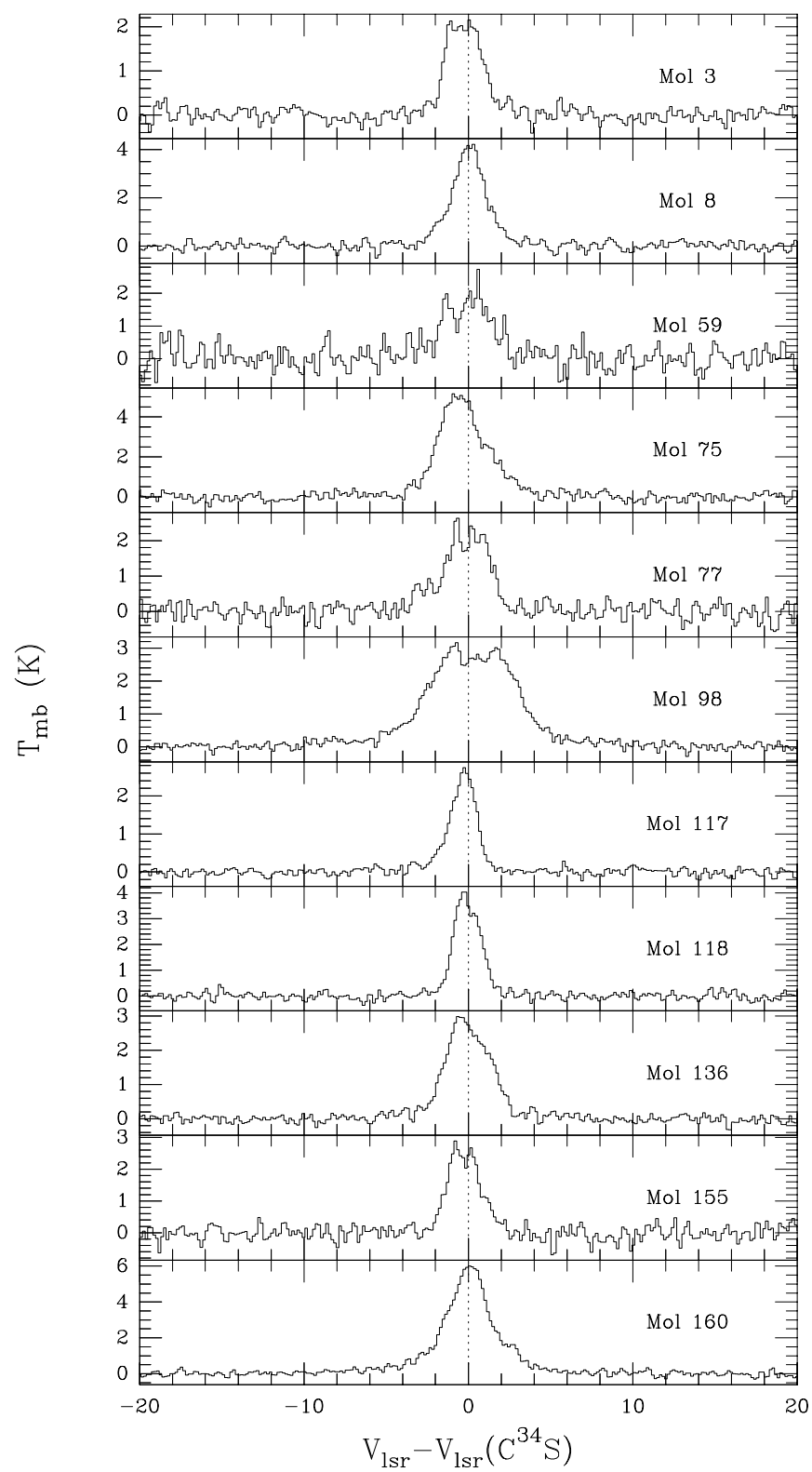

Fig. 6. c) As a), but for the $\operatorname{CS}(3-2)$ spectra

shoulder (blue or red). The dip (or the start of the shoulder) is at the velocity of the $\mathrm{C}^{34} \mathrm{~S}$ line, which is also the velocity of the peak of the $\mathrm{C}^{18} \mathrm{O}$ and $\mathrm{CH}_{3} \mathrm{C}_{2} \mathrm{H}$ lines, and that of the $\mathrm{NH}_{3}(1,1)$ and $(2,2)$ lines (Molinari et al. 1996). At all observed positions, the $\mathrm{HCO}^{+}$spectrum shows only very weak emission on the red side of this dip. In Fig. 15 we compare the spectra of ${ }^{13} \mathrm{CO}$ and $\mathrm{HCO}^{+}$with the ${ }^{12} \mathrm{CO}$ spectra we obtained with the NRAO $12-\mathrm{m}$ (beam $\left.F W H P \sim 29^{\prime \prime}\right)$. From this comparison it seems likely that the ${ }^{13} \mathrm{CO}$ line profiles are the result of the superposition of two components, rather than being due to self-absorption. In the $\mathrm{HCO}^{+}$spectra, the bluer component clearly is the dominant one. The ${ }^{12} \mathrm{CO}$ spectra show broad emission at velocities $>80 \mathrm{~km} \mathrm{~s}^{-1}$, some of which is also seen in ${ }^{13} \mathrm{CO}$ (see Fig. 16a), and is probably the result of additional emission components. On the blue side however

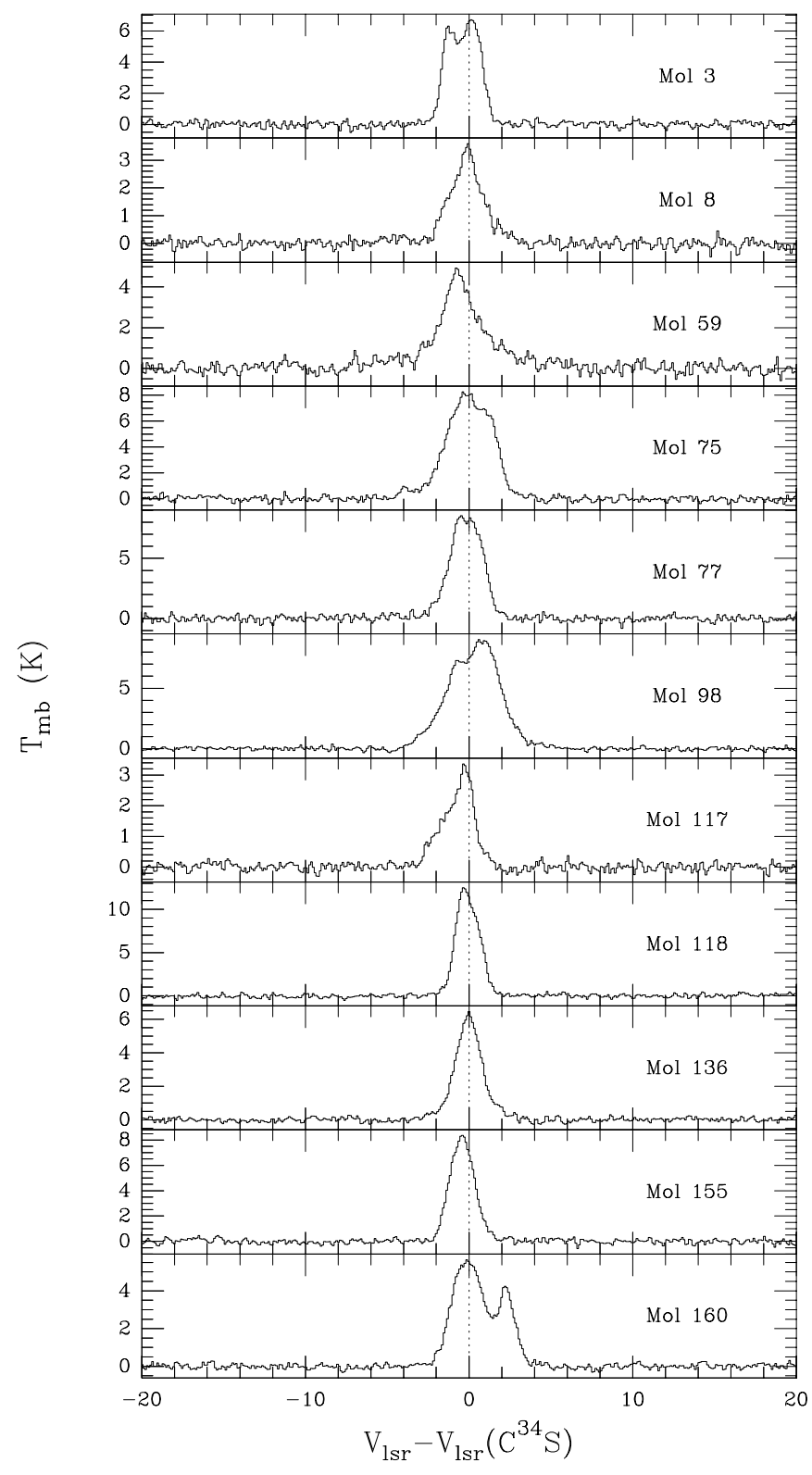

Fig. 6. d) As a) but for the $\mathrm{C}^{18} \mathrm{O}(2-1)$ spectra

$\left(V_{\mathrm{lsr}} \lesssim 74 \mathrm{~km} \mathrm{~s}^{-1}\right)$, the ${ }^{12} \mathrm{CO}$ spectra are much steeper. At these velocities some emission is also present in the spectra of the molecules observed at IRAM (see Figs. 1e, and 16), and may be outflow emission associated with the component on the blue side of the dip; an eventual red component of this outflow is obscured by the emission of the other components redward of the dip.

To isolate the various components, we have integrated the line profiles over appropriately chosen velocity intervals; the results are shown in Fig. 16. From the dominance of the $75 \mathrm{~km} \mathrm{~s}^{-1}$ component of $\mathrm{HCO}^{+}$, and the fact that it peaks at or very near the IRAS source and submm peak, we conclude that the emission in the interval $\sim 74-76 \mathrm{~km} \mathrm{~s}^{-1}$ is likely to represent the associated component. However, also the emission between 76 and $80 \mathrm{kms}^{-1}$ peaks at that location, most clearly seen in 


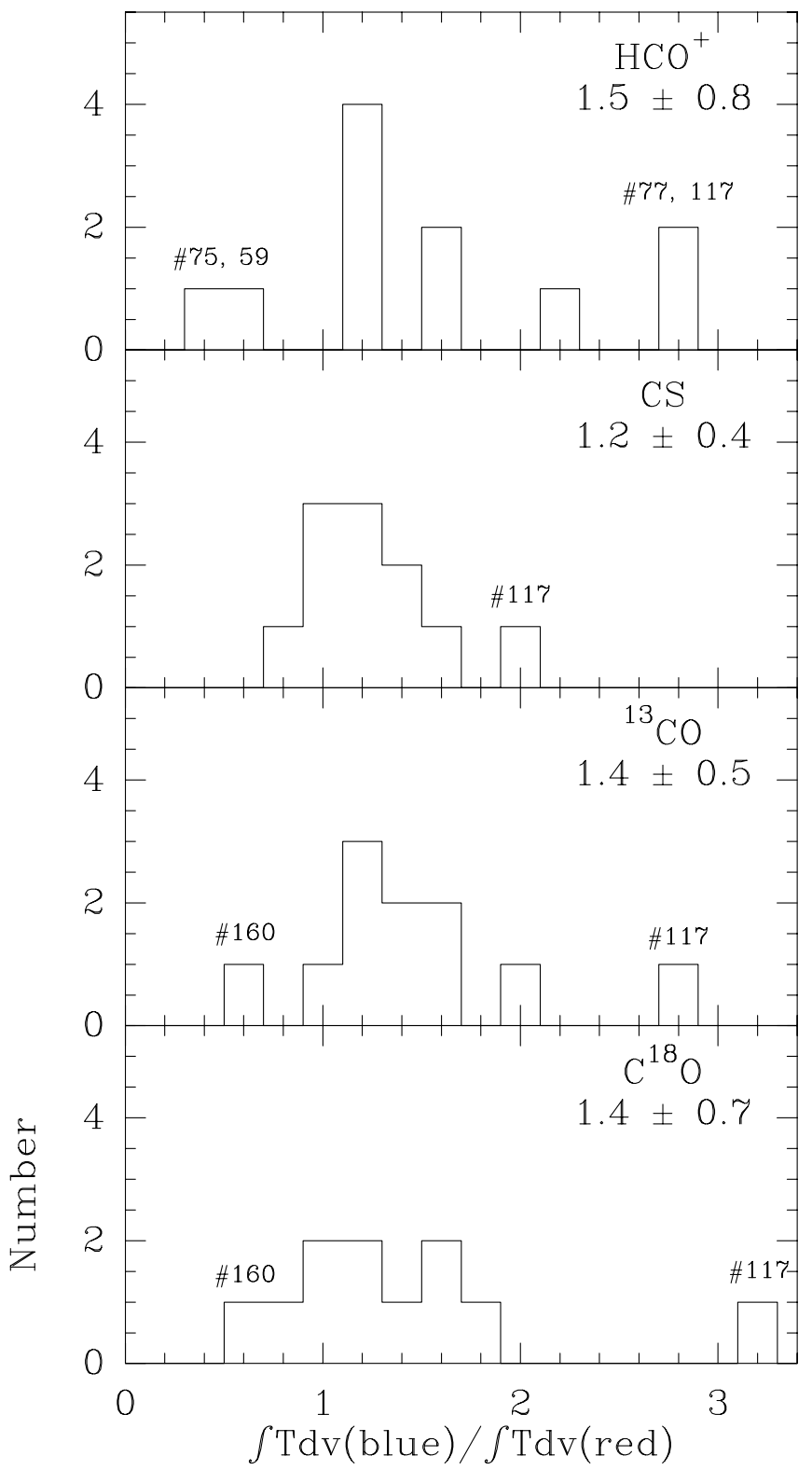

Fig. 7. Distribution of the ratio of $\int T \mathrm{~d} v$ over the blue and red (with respect to the $\mathrm{C}^{34} \mathrm{~S}$ velocity) parts of the spectra at the peak positions, for the indicated tracers. For each molecule, the average ratio is indicated, as are the source numbers of the extreme values

the distribution for the density tracers $\mathrm{C}^{18} \mathrm{O}$ and $\mathrm{CS}$ (Fig. 16c), as does the emission between 71 and $74 \mathrm{~km} \mathrm{~s}^{-1}$.

\section{6. $\mathrm{Mol} 98$}

Towards this region we found an $\mathrm{H}_{2} \mathrm{O}$ maser (Palla et al. 1991), while a $6.7 \mathrm{GHz}$ methanol maser was detected by MacLeod et al. (1998). As they mention, methanol masers are unique indicators of massive star-forming regions, because unlike water- and hydroxyl masers, methanol masers have not been found towards stars of spectral type later than B2. These latter authors also detected circularly polarized $1665 \mathrm{MHz} \mathrm{OH}$ maser emission. Molinari et al. (1998a) found no 2 and $6-\mathrm{cm}$ radio continuum

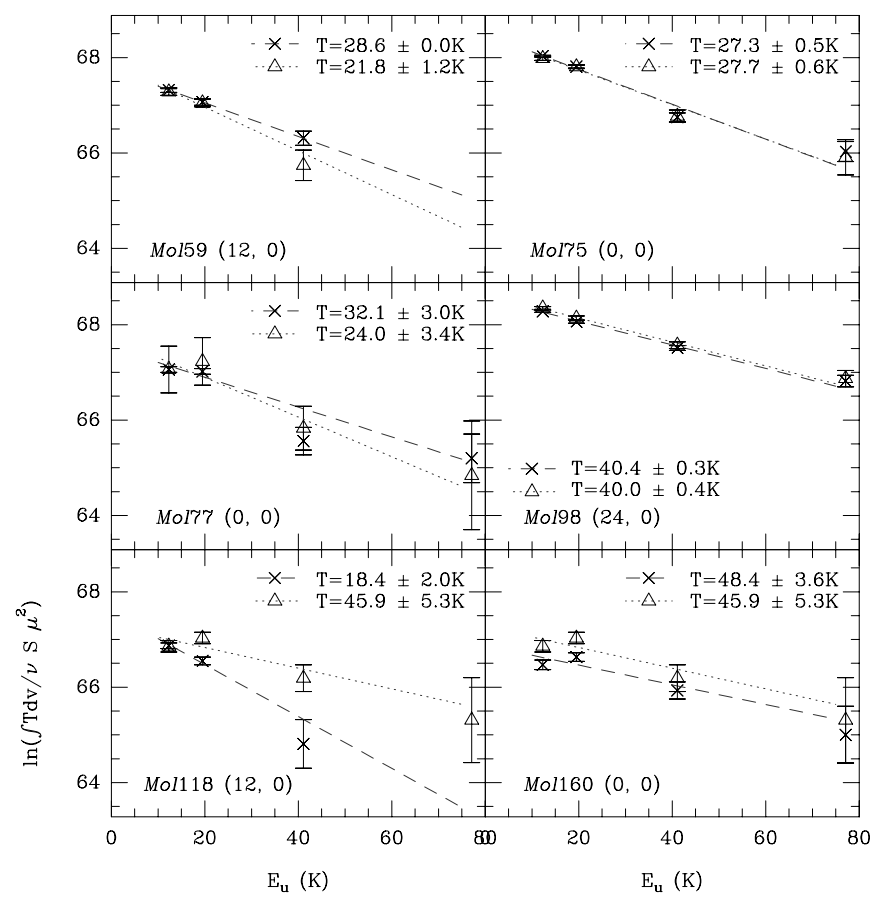

Fig. 8. Boltzmann plots, constructed from the $\mathrm{CH}_{3} \mathrm{C}_{2} \mathrm{H}(6-5)$ observations. The results from the high- $\left(0.23 \mathrm{~km} \mathrm{~s}^{-1}\right)$ and lowresolution $\left(2.92 \mathrm{~km} \mathrm{~s}^{-1}\right)$ spectra are shown as crosses and triangles, respectively. The dashed and dotted lines are weighted least-squares fits through the data points. The sources are identified in the panels, where also the derived $T_{\text {kin }}$ is given. Full results are presented in Table 7

Table 8. Adopted $T_{\mathrm{ex}}$ and estimated masses of clumps associated with the IRAS sources

\begin{tabular}{rrrl}
\hline $\begin{array}{r}\text { Mol } \\
\#\end{array}$ & $\begin{array}{r}T_{\text {ex }} \\
(\mathrm{K})\end{array}$ & $\begin{array}{r}\text { Mass }^{1} \\
\left(M_{\odot}\right)\end{array}$ & Associated velocity component \\
\hline 3 & 35 & 2140 & blue $\left(V_{\mathrm{lsr}}<-50.85\right)$ \\
& & 534 & red $\left(V_{\mathrm{lsr}}>-50.85\right)$ \\
8 & 25 & 238 & red $\left(V_{\mathrm{lsr}} \approx-25.5\right)$ \\
59 & 15 & 65 & red $\left(114.3<V_{\mathrm{lsr}}<117.0\right)$ \\
75 & 25 & 504 & blue $\left(55.0<V_{\mathrm{lsr}}<58.6\right)$ \\
77 & 25 & 187 & blue $\left(73.8<V_{\mathrm{lsr}}<76.3\right)$ \\
98 & 35 & 424 & $\left(V_{\mathrm{lsr}} \approx 57.0\right)$ \\
117 & 40 & 1124 & red $\left(-36.9<V_{\mathrm{lsr}}<-33.0\right)$ \\
118 & 35 & 93 & blue $\left(6.5<V_{\mathrm{lsr}}<8.5\right)$ \\
136 & 30 & 370 & $\left(V_{\mathrm{lsr}} \approx-46.8\right)$ \\
155 & 30 & 968 & blue $\left(V_{\mathrm{lsr}}<-51.1\right)$ \\
160 & 35 & 621 & main $\left(V_{\mathrm{lsr}} \approx-50.2\right)$ \\
& & &
\end{tabular}

1 Lower limit, derived from the average $\mathrm{H}_{2}$ column density, estimated from the ${ }^{13} \mathrm{CO}$ emission above the $F W H M$ level, and the beam-corrected area of the emission.

emission associated with this IRAS source. However, in recent VLA-D observations at 3.6-cm (Molinari et al., in preparation) continuum emission with a peak flux density $\sim 1 \mathrm{mJy} /$ beam was detected at $\sim\left(+10^{\prime \prime},+14^{\prime \prime}\right)$ from the present map center. Recent SCUBA observations (Molinari et al., in preparation) at $450 \mu \mathrm{m}$ show a submm peak at $\sim\left(+30^{\prime \prime},+3^{\prime \prime}\right)$ from the present map center. 


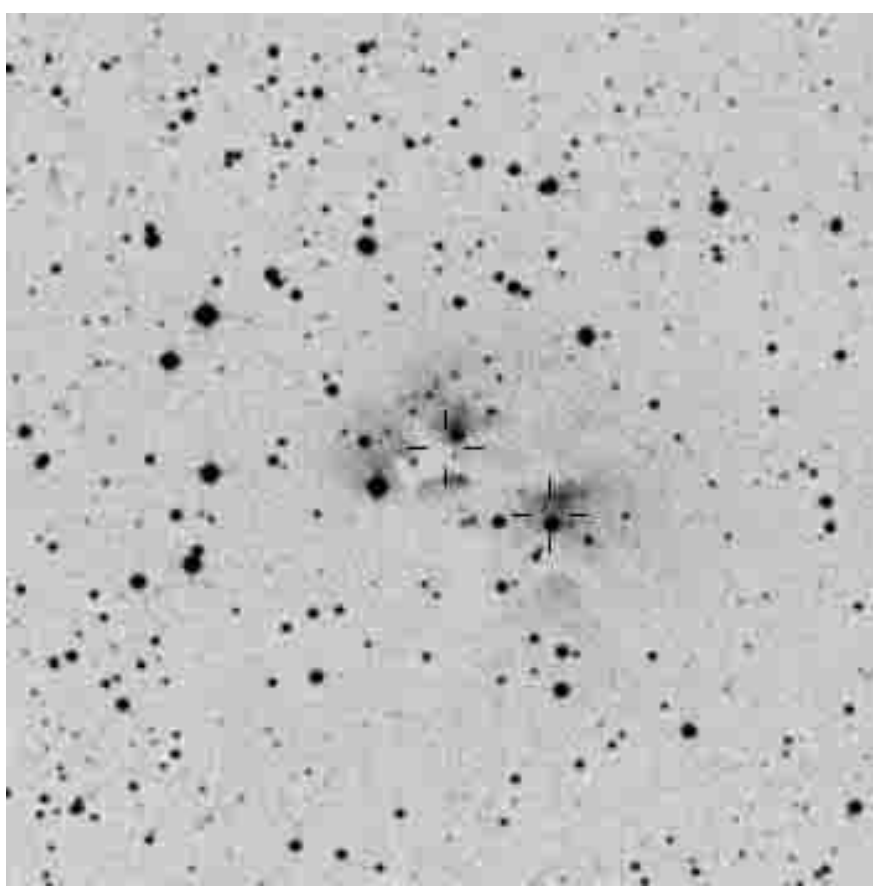

Fig. 9. Optical image (Digital Sky Survey) of a $10^{\prime} \times 10^{\prime}$ region around $\mathrm{Mol} 3 . \mathrm{N}$ is up, $\mathrm{E}$ is left; the $(0,0)$ position of the mapped region is indicated by the easternmost cross. The westernmost cross indicates the location of the 6 -cm continuum peak detected by White \& Gee 1986 (GN0042.0-2; see text)

We note that this is quite different from the offset of the sub-mm peak as given in Molinari et al. (2000) $\left(-10^{\prime \prime},-10^{\prime \prime}\right.$ from the IRAS position, i.e. $-4^{\prime \prime},-10^{\prime \prime}$ from the map center), which was however only based on a 5point cross observation, and hence less trustworthy.

The present molecular observations show a quite compact core, even in ${ }^{13} \mathrm{CO}$, with a peak $\sim 25^{\prime \prime} \mathrm{E}$ of the IRAS source (Fig. 5f). The sum of all ${ }^{13} \mathrm{CO}$ spectra of this source shows two absorption dips, at $V_{\mathrm{lsr}} \sim 62 \mathrm{~km} \mathrm{~s}^{-1}$ and $\sim 70 \mathrm{~km} \mathrm{~s}^{-1}$, indicating that here the offset position $\left(\Delta \alpha, \Delta \delta=-1800^{\prime \prime}, 0^{\prime \prime}\right.$ from the center of the map) was not far enough from the molecular cloud in which the object is embedded. We have tried to correct for this by summing all ${ }^{13} \mathrm{CO}$ spectra with narrow $\left(V_{\mathrm{lsr}} \lesssim 60 \mathrm{~km} \mathrm{~s}^{-1}\right)$ emission, fitting Gaussians to the dips (which have a strength of $\sim 1-1.5 \mathrm{~K}$ ), and adding those components to all ${ }^{13} \mathrm{CO}$ spectra in the map. Unfortunately this does not correct for any absorption that might be present in the main line, but inspection of the spectra taken at positions without ${ }^{13} \mathrm{CO}$ emission $\left(\Delta \alpha=96^{\prime \prime}\right)$ indicates that any such absorption will be $\lesssim 2.4 \mathrm{~K}\left(\sim 2 \sigma_{\text {rms }}\right.$ in the individual spectra at those positions).

The channel maps for this source indicate that there is only one emission component associated with the object (the ${ }^{12} \mathrm{CO}$ spectra in the NRAO $12-\mathrm{m}$ map, that covers an area of about $300^{\prime \prime} \times 300^{\prime \prime}$, show many emission components, but near Mol 98 the dominating one is that between $\sim 50-60 \mathrm{~km} \mathrm{~s}^{-1}$ ); the non-Gaussian profiles seen in

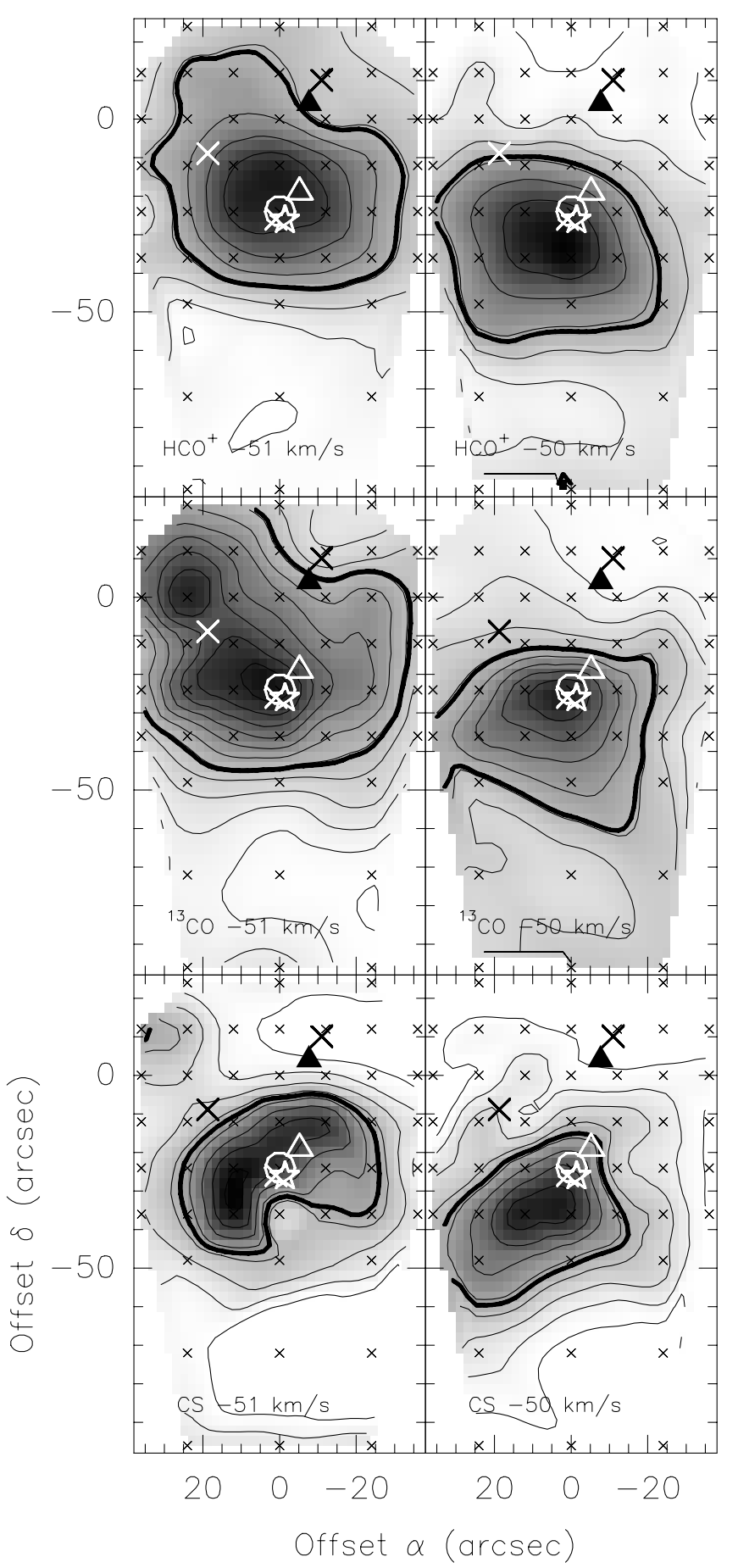

Fig. 10. Mol 3 data. Integrated emission of the red and blue emission (with respect to $-50.85 \mathrm{~km} \mathrm{~s}^{-1}$ ) for the indicated molecules. Red and blue integration limits are $-55,-48$ $\left(\mathrm{HCO}^{+}\right),-54,-48.5\left({ }^{13} \mathrm{CO}\right)$, and $-54,-48(\mathrm{CS})$. The symbols have the following meaning: filled triangle - IRAS source; open circle - molecular peak; open triangle $-850 \mu \mathrm{m}$ peak; open star $-\mathrm{H}_{2} \mathrm{O}$ maser position; big crosses - VLA-D array $3.6 \mathrm{~cm}$ peaks. Small crosses indicate the observed positions. Contour values are $0.3(0.5) 3.9,0.2(0.5) 3.2 \mathrm{Kkm} \mathrm{s}^{-1}$ $\left(\mathrm{HCO}^{+}\right.$blue, red); 5.5(3)41.5, 2(3)31 $\mathrm{Kkm} \mathrm{s}^{-1}\left({ }^{13} \mathrm{CO}\right.$ blue, red); $0.2(0.4) 3.2,0.2(0.5) 4.2 \mathrm{Kkm} \mathrm{s}^{-1}$ (CS blue, red); the thick contours indicate the $F W H M$ level 


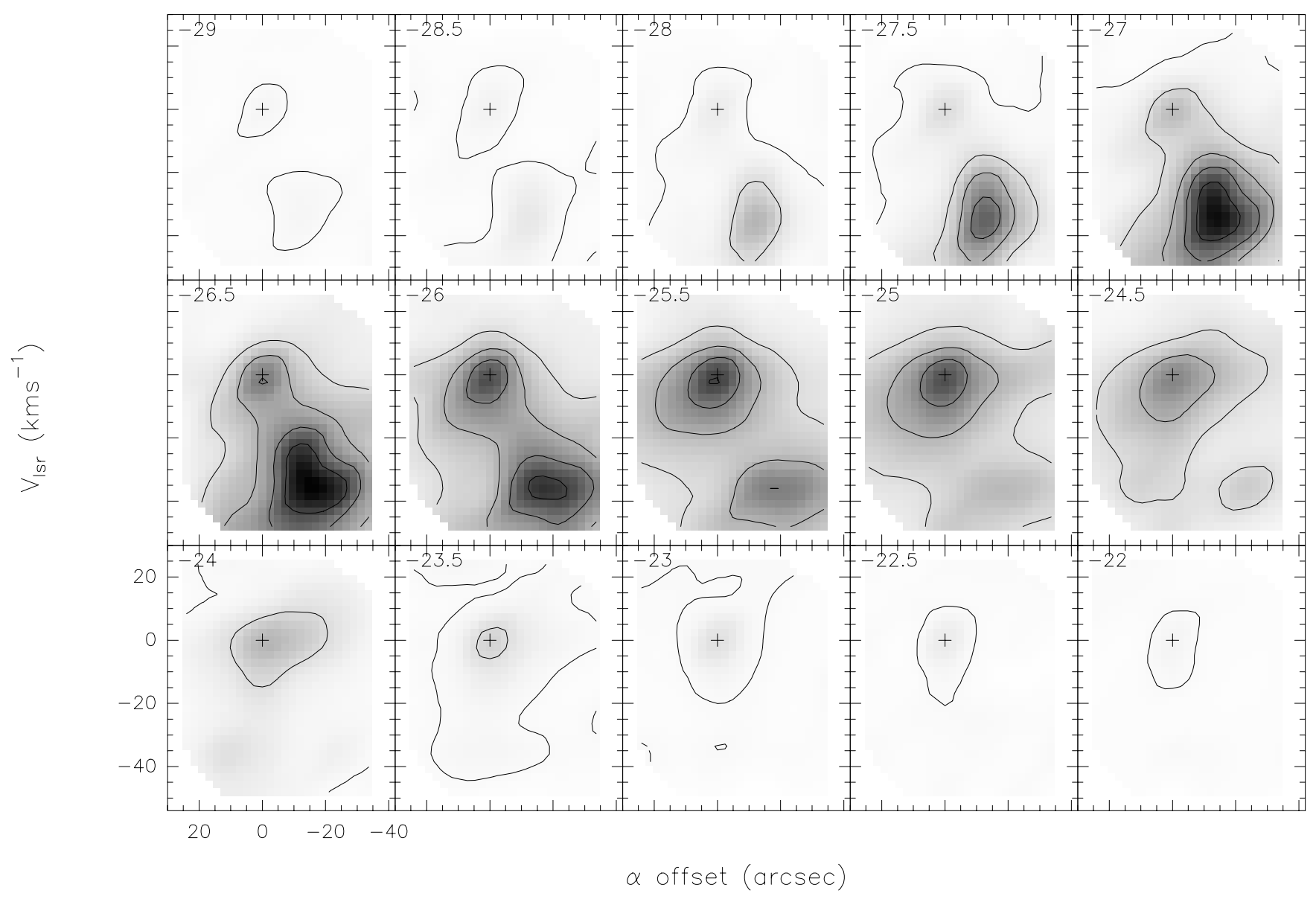

Fig. 11. Mol 8 data. Integrated emission of the ${ }^{13} \mathrm{CO}$ line, shown in steps of $0.5 \mathrm{~km} \mathrm{~s}^{-1}$. The cross indicates the position of the sub-mm peak ( $\approx$ IRAS source position). Contours $0.5(4) 17 \mathrm{Kkm} \mathrm{s}^{-1}$. The $\mathrm{HCO}^{+}$and $\mathrm{CS}$ emission show the same behaviour, and are not shown

Fig. 1f (flat top or shoulder) may be caused by saturation or self-absorption.

The spectra in Fig. If show non-Gaussian wings to all profiles, even that of $\mathrm{C}^{34} \mathrm{~S}$. In Fig. 17 we show the distribution of the integrated emission over the line wings. Note that $\mathrm{HCO}^{+}$has a relatively strong blue wing, which extends up to $15 \mathrm{kms}^{-1}$ from the velocity of the bulk of the molecular material. The outflow is bipolar in all 3 lines shown, and has the center of the line connecting the lobes at offset $\approx\left(30^{\prime \prime}, 5^{\prime \prime}\right)$, which puts it at the location of the $450 \mu \mathrm{m}$ peak.

\section{7. $\mathrm{Mol} 117$}

On the DSS some faint diffuse emission is visible at the location of this object. Rather weak $\mathrm{NH}_{3}(1,1)$ emission was detected (Molinari et al. 1996) at $V_{\mathrm{lsr}} \approx-36.4 \mathrm{~km} \mathrm{~s}^{-1}$, which is also the velocity at which $\mathrm{HCO}^{+}(4-3)$ (see Fig. 2) is found. No $6.7 \mathrm{GHz}$ methanol maser was detected by MacLeod et al. (1998). The molecules observed at IRAM have their peak emission at $V_{\mathrm{lsr}} \approx-35.7 \mathrm{~km} \mathrm{~s}^{-1}$ (Fig. $1 \mathrm{~g}$ and Table 3). Our $\mathrm{C}^{18} \mathrm{O}(2-1)$ NRAO $12-\mathrm{m}$ data show (at position $0^{\prime \prime}, 0^{\prime \prime}$, and with a $29^{\prime \prime}$ beam) a double-peaked

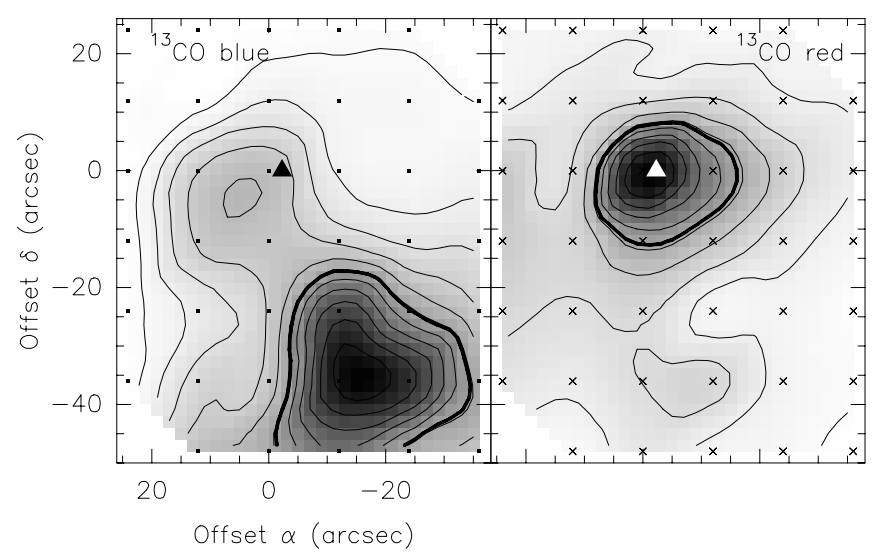

Fig. 12. Mol 8 data. Emission under the ${ }^{13} \mathrm{CO}$ lines, integrated over the blue and red components (separated by Gaussian fits). Contour values are 2(4) $45 \mathrm{Kkm} \mathrm{s}^{-1}$ (blue) and 2(4) $38 \mathrm{Kkm} \mathrm{s}^{-1}$ (red), respectively. The thick contours indicate the $F W H M$ level. The triangle represents the IRAS position

profile, with components at -37.9 and $-35.9 \mathrm{kms}^{-1}$, and a dip at $V_{\mathrm{lsr}} \approx-37 \mathrm{~km} \mathrm{~s}^{-1}$; the redder component therefore coincides with the velocities of the peak emission found at IRAM, while the $\mathrm{NH}_{3}$ velocity lies closer 


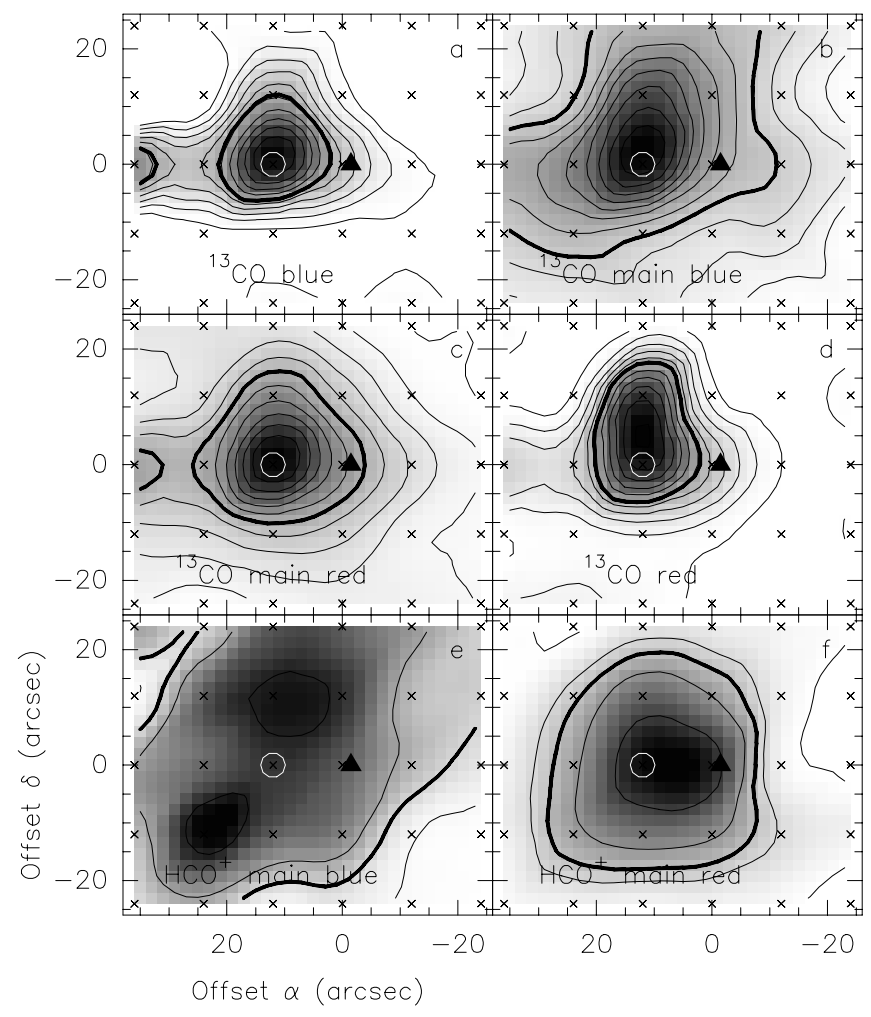

Fig. 13. Mol 59 data. Emission in the ${ }^{13} \mathrm{CO}$ (top 4 panels) and $\mathrm{HCO}^{+}$(bottom 2 panels) lines, integrated over different velocity intervals: a) $105-111 \mathrm{~km} \mathrm{~s}^{-1}$ [lowest contour 0.5 , step $1 \mathrm{Kkm} \mathrm{s}^{-1}$ ]; b) $111-114.3 \mathrm{~km} \mathrm{~s}^{-1}\left[5.5,1.5 \mathrm{Kkm} \mathrm{s}^{-1}\right]$; c) $114.3-117 \mathrm{~km} \mathrm{~s}^{-1}\left[1.5,1.5 \mathrm{Kkm} \mathrm{s}^{-1}\right]$; d) $117-122 \mathrm{~km} \mathrm{~s}^{-1}$ $\left[0.5,1 \mathrm{Kkm} \mathrm{s}^{-1}\right]$; e) $111-114.3 \mathrm{~km} \mathrm{~s}^{-1}\left[0.5,0.5 \mathrm{Kkm} \mathrm{s}^{-1}\right]$; f) $114.3-117 \mathrm{~km} \mathrm{~s}^{-1}\left[0.5,0.5 \mathrm{Kkm} \mathrm{s}^{-1}\right]$. Thick contours in each panel indicate the half-maximum level. The IRAS position is indicated by the triangle, the white circle is the position where longer integrations were made. Small crosses are the observed positions

to the dip in the NRAO $\mathrm{C}^{18} \mathrm{O}(2-1)$ spectrum. However, also the IRAM spectra show double-peaked profiles, as illustrated by the ${ }^{13} \mathrm{CO}$ line at $\left(-12^{\prime \prime}, 0^{\prime \prime}\right)$ in Fig. $1 \mathrm{~g}$. In fact, at most positions we find that the line profiles have shoulders or double peaks, with the dip always at the same velocity. We therefore analyze the data in terms of the superposition of two emission components. The components are separated by integrating between -40 and $-36.9 \mathrm{~km} \mathrm{~s}^{-1}$ (blue) and -36.9 and $-33 \mathrm{kms}^{-1}$ (red) for ${ }^{13} \mathrm{CO}$ and $\mathrm{HCO}^{+}$, and -39.5 and $-36.3 \mathrm{kms}^{-1}$ (blue) and -36.3 and $-33 \mathrm{~km} \mathrm{~s}^{-1}$ (red) for CS. The distributions of the integrated emission are shown in Fig. 18. Because the red peak seems to be the dominant one, we assume this is the associated component.

\section{8. $\mathrm{Mol} 118$}

This is the object with the smallest kinematical distance $(1.6 \mathrm{kpc})$ in the sample. The $(0,0)$ position of the maps, and the IRAS source (at offset $-3 !^{\prime \prime} 7,0^{\prime \prime}$ ) are located in

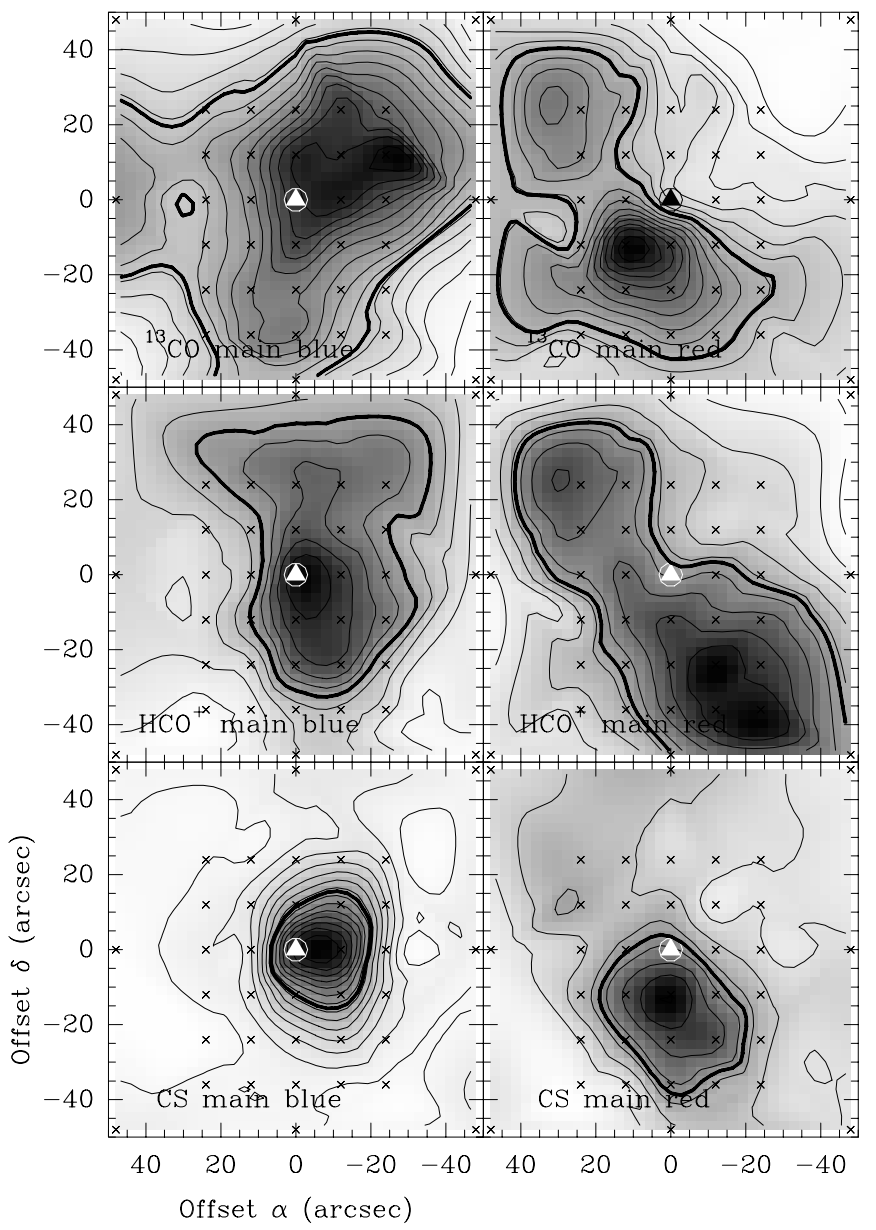

Fig. 14. Mol 75 data. Emission in the ${ }^{13} \mathrm{CO}$ (top 2 panels), $\mathrm{HCO}^{+}$(middle 2 panels), and CS (bottom 2 panels) lines, integrated over the blue and red parts of the emission (see text). Thick contours in each panel indicate the half-maximum level. The IRAS position is indicated by the triangle, the white circle is the position where longer integrations were made. Small crosses are the observed positions. Contours: ${ }^{13} \mathrm{CO}$ blue $8(2) 37 \mathrm{Kkm} \mathrm{s}^{-1}$, red $4(2) 36 ; \mathrm{HCO}^{+}$blue $0.2(0.2) 2$, red 0.2(0.8)7.4; CS blue 1(1)15, red 1(1)7

a dark cloud, which is surrounded by diffuse emission. No 6.7 GHz methanol maser was detected by MacLeod et al. (1998).

The spectra shown in Fig. 1h are non-Gaussian, and as evidenced by the $\mathrm{C}^{34} \mathrm{~S}$ spectrum, and the superimposed ${ }^{13} \mathrm{CO}$ and $\mathrm{C}^{18} \mathrm{O}$ spectra at offset $\left(12^{\prime \prime}, 12^{\prime \prime}\right)$, this is due to the presence of two velocity components. In addition, emission in the wings of the profiles is visible, which may be due to outflow, or to additional components. These wings are also visible in the NRAO $\mathrm{CO}(2-1)$ spectra. We separate the two main ("blue" and "red") components by integrating over the following velocity intervals: $6.5-8.5-$ $9.4 \mathrm{~km} \mathrm{~s}^{-1}\left({ }^{13} \mathrm{CO}\right) ; 6.5-8.4-9.4 \mathrm{~km} \mathrm{~s}^{-1}\left(\mathrm{HCO}^{+}\right) ; 6.8-8.2-$ $9.2 \mathrm{kms}^{-1}$ (CS). These integration limits omit the wing emission. The distribution of the emission in each component is shown in Fig. 19. The $\mathrm{NH}_{3}$ velocity is $7.8 \mathrm{~km} \mathrm{~s}^{-1}$, that of $\mathrm{CH}_{3} \mathrm{C}_{2} \mathrm{H}$ is 7.9 , and thus coinciding with the (more 


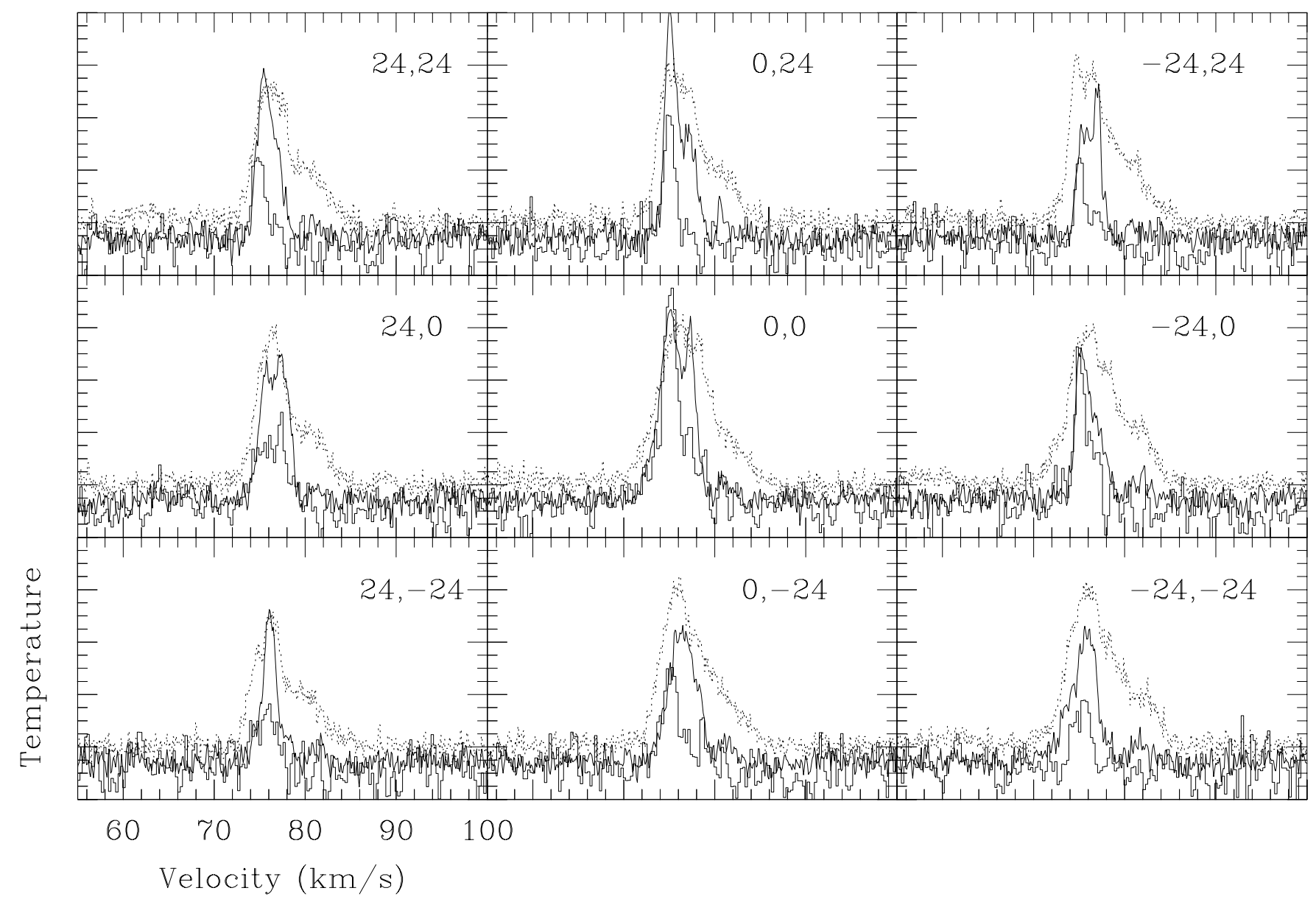

Fig. 15. Mol 77 data. Comparison of line profiles of ${ }^{12} \mathrm{CO}$ (dashed), ${ }^{13} \mathrm{CO}$ (drawn), and $\mathrm{HCO}^{+}$(histogram), in the central part of the cloud. The vertical scale is temperature, but scaled such as to make all lines clearly visible. The NRAO $12-\mathrm{m}{ }^{12} \mathrm{CO}$ spectra were actually taken on a $29^{\prime \prime}$ grid, rather than $24^{\prime \prime}$. The beam of the ${ }^{12} \mathrm{CO}$ observations $\left(\sim 29^{\prime \prime}\right)$ is comparable to that of the IRAM 30-m HCO ${ }^{+}\left(\sim 27^{\prime \prime}\right)$; the ${ }^{13} \mathrm{CO}$ observations were made with a $\sim 11^{\prime \prime}$ beam. These plots suggest that the dip/shoulder in the IRAM spectra is probably due to the superposition in velocity of 2 components

intense) blue component, which we will take as the associated one.

\section{9. $\mathrm{Mol} 136$}

Inspection of the data reveal that there is only one emission component detected towards this source. The line profiles are non-Gaussian, being skewed towards the blue (see Fig. 1i). This is also seen in the ${ }^{12} \mathrm{CO}(1-0)$ line profile (Wouterloot \& Brand 1989; source WB89 93). Low-level emission is also seen extending over a few $\mathrm{kms}^{-1}$ from the central line velocity; the distribution of the integrated emission in these wings is shown in Fig. 20. The blue emission peaks near the IRAS source position (which is also the peak of the sub-mm emission), while the red component has its maximum more to the West.

Although Molinari et al. (1998a) did not detect associated radio continuum emission (VLA, 2 and 6-cm), deeper observations at 3.6-cm with the VLA D-array by Molinari et al. (in preparation), reveal diffuse emission (peak flux density $\sim 6.2 \mathrm{mJy} /$ beam) just NE of the IRAS source.

\subsection{0. $\mathrm{Mol} 155$}

The emission of especially ${ }^{13} \mathrm{CO}$ and $\mathrm{HCO}^{+}$is quite extended in this source (see Fig. 5j). The line profiles are similar to those in Mol 77 (cf. Figs. 1e and j): The profiles of ${ }^{13} \mathrm{CO}$ and $\mathrm{HCO}^{+}$show a dip or a shoulder everywhere, while for the latter molecule the blue component is much stronger than the red one at every observed position. The dip or shoulder is at $V_{\mathrm{lsr}} \sim-51.1,-50.7$ and $-51.0 \mathrm{~km} \mathrm{~s}^{-1}$ for ${ }^{13} \mathrm{CO}, \mathrm{HCO}^{+}$, and $\mathrm{CS}$, respectively. The velocity of the $\mathrm{NH}_{3}(1,1)$ line is at $-51.5 \mathrm{~km} \mathrm{~s}^{-1}$, thus in the velocity range of the blue component. We separate the two emission components by integrating blue- and redwards of the dip. The resulting distributions of the integrated emission are shown in Fig. 21. The dominance of the $\mathrm{HCO}^{+}$blue component is clearly visible there. We note that towards this source observations of $\mathrm{SiO}(2-1)$ (Harju et al. 1998) have yielded no detections. 


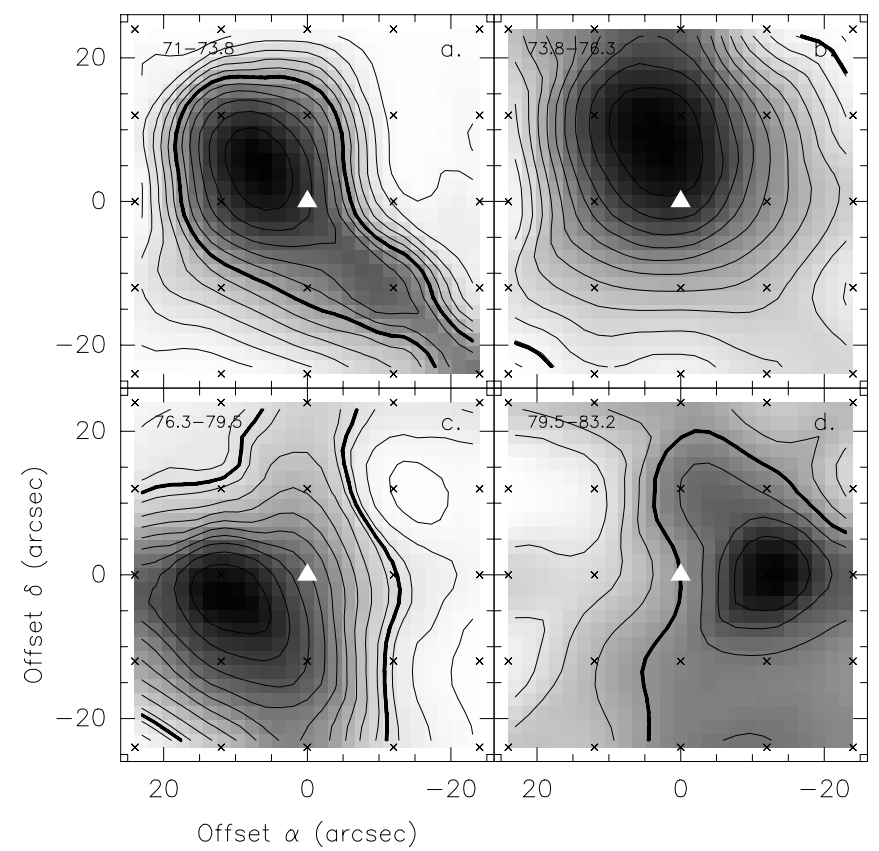

Fig. 16. a) Distribution of $\int T \mathrm{~d} v$ of the ${ }^{13} \mathrm{CO}$ emission towards Mol 77, integrated over the velocity ranges indicated in the panels. In each panel an individual velocity component is isolated. Thick contours in each panel indicate the halfmaximum level. The IRAS position is indicated by the triangle, and is identical to the position where longer integrations were made. Small crosses are the observed positions. Contours: $0.5(0.5) 6 \mathrm{Kkm} \mathrm{s}^{-1}$ (panel a)); 10(1)22 b); 6(1)19 c); 0.5(0.5)4 d)

\subsection{Mol 160}

This source has been observed and discussed extensively by Molinari et al. (1998b), who concluded that this is a very good candidate massive Class 0 object. Inspection of the line profiles at the peak position (Fig. 1k; especially $\mathrm{C}^{18} \mathrm{O}$ ) strongly suggests the presence of two velocity components. Individual ${ }^{13} \mathrm{CO}$ spectra indicate that there might be more than two components; we also note that the dip, or shoulder, in these spectra is not always at the same velocity. This may be an indication of the presence of a velocity gradient in the ${ }^{13} \mathrm{CO}$ emission, and separating the contribution of the various components by integrating over fixed velocity intervals is hazardous. We have therefore performed Gaussian fits to the profiles of ${ }^{13} \mathrm{CO}, \mathrm{HCO}^{+}$, and CS (even though for the latter two we do not see a shift in the position of the dip (if at all) with position). Based on the $V_{\mathrm{lsr}}$ of the $\mathrm{NH}_{3}(1,1)$ and $\mathrm{CH}_{3} \mathrm{C}_{2} \mathrm{H}(6-5)$ lines ( -50.0 and $-50.45 \mathrm{~km} \mathrm{~s}^{-1}$ respectively), we identify the main emission component. After subtraction of the other Gaussian components, we integrated the spectra; the resulting distributions of the emission are shown in Fig. 22. The emission peaks lie close to the $3.4-\mathrm{mm}$ continuum peak found with OVRO by Molinari et al. (1998b).

Figure 23 shows a series of Right Ascension versus $V_{\mathrm{lsr}}$ plots for the ${ }^{13} \mathrm{CO}$ line, at the Declination offsets labeled in each panel. Especially at positive Declination offsets the

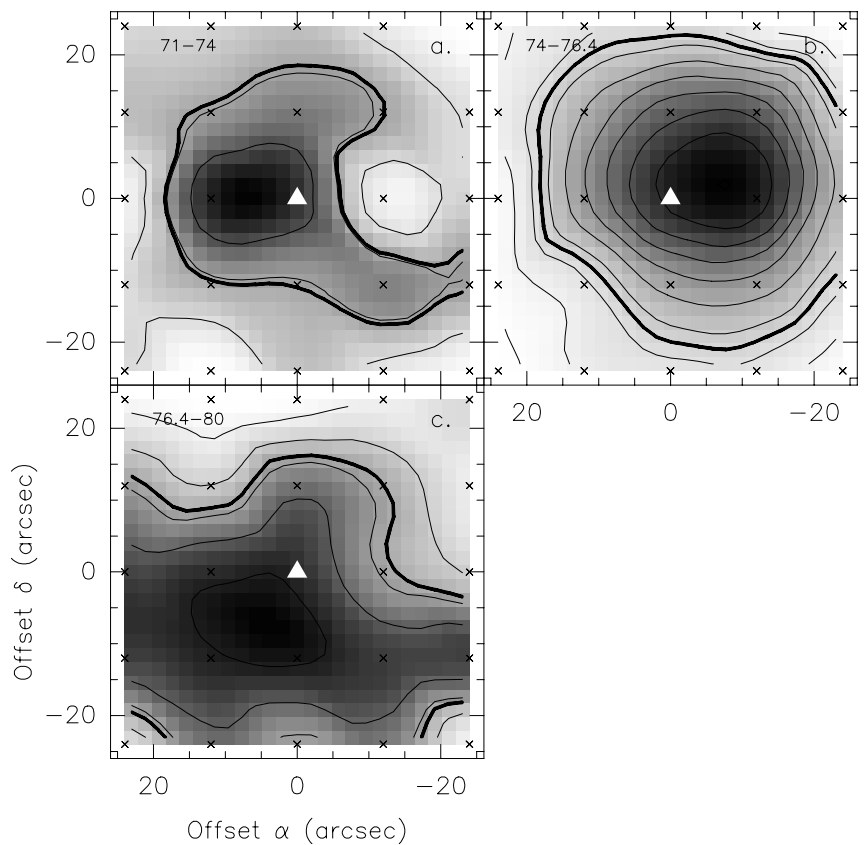

Fig. 16. b) As a) but for $\mathrm{HCO}^{+}$. Contours: $0.2(0.2) 0.8 \mathrm{Kkm} \mathrm{s}^{-1}$ (panel a)); 0.8(0.2)2.6 b); 0.2(0.2)1.2 c)

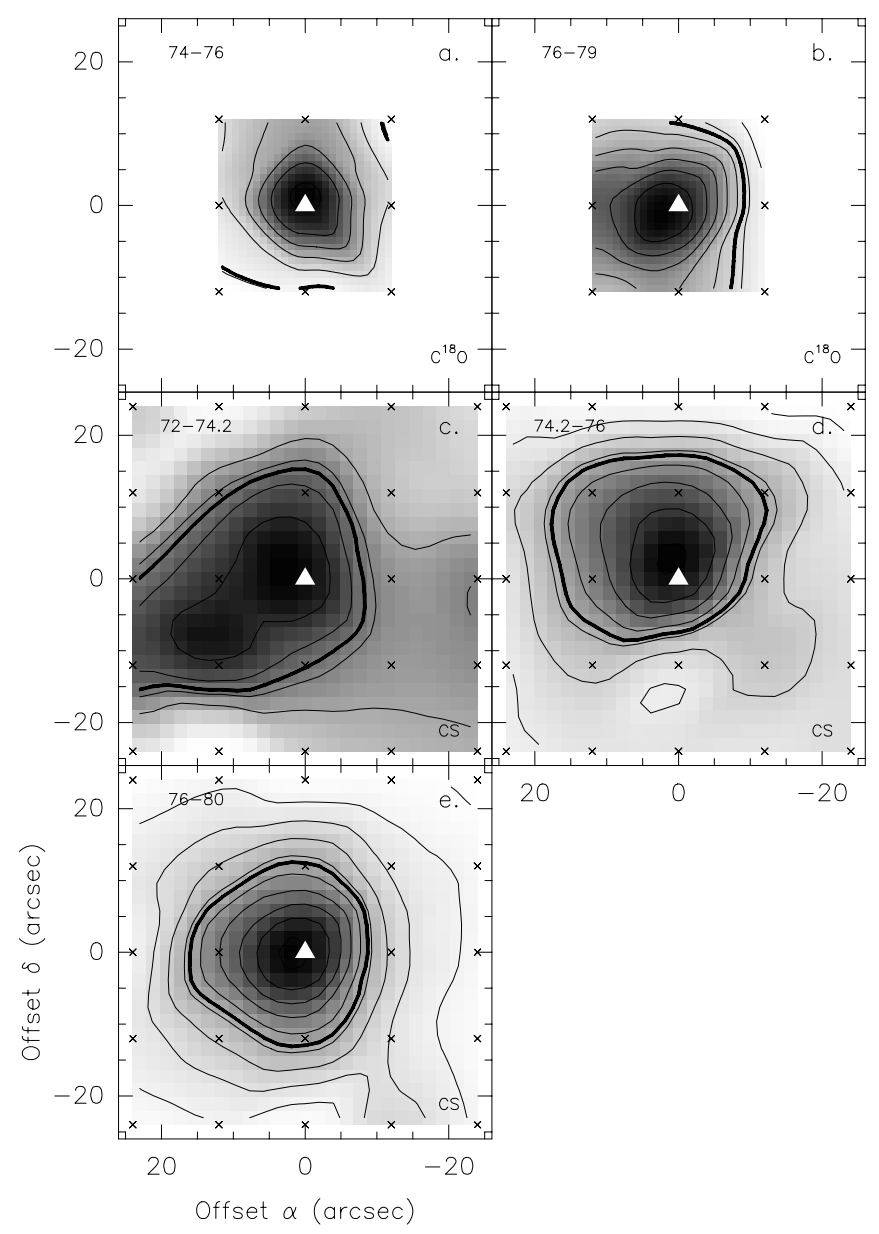

Fig. 16. c) As a) but for $\mathrm{C}^{18} \mathrm{O}($ panels a, b)) and CS (panels ce)). Contours: $5(1) 10 \mathrm{Kkm} \mathrm{s}^{-1}$ (panel a)); 3(1)11 b); 0.2(0.2)1 c); $0.2(0.4) 3.4 \mathbf{d}) ; 0.2(0.4) 4 \mathbf{e})$ 


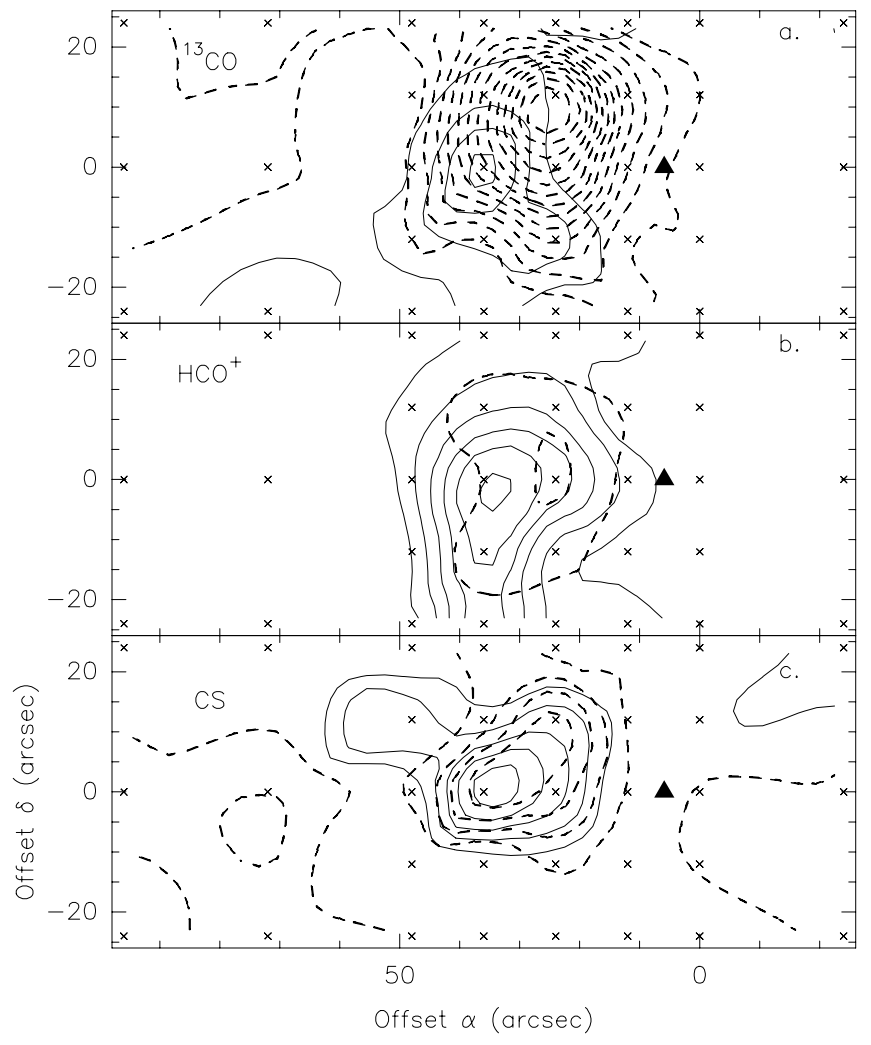

Fig. 17. Mol 98 data. Emission in the wings of the profiles of $\left.\left.{ }^{13} \mathrm{CO} \mathbf{a}\right), \mathrm{HCO}^{+} \mathbf{b}\right)$, and $\left.\mathrm{CS} \mathbf{c}\right)$. Drawn and dashed contours refer to blue and red wings, respectively. Integrated velocity interval and contour values (blue, red: range, low(step)max) are: 52-54: 1(1)4, 60-65: 1(1)14 $\mathrm{Kkm} \mathrm{s}^{-1}$ a); 45-54: 0.5(0.5)3, 60-64: $0.5(0.5) 1 \mathrm{Kkm} \mathrm{s}^{-1}$ b); 48-54: $0.5(0.5) 2.5, \quad 61-65$ : $\left.0.5(0.5) 2.5 \mathrm{Kkm} \mathrm{s}^{-1} \mathbf{c}\right)$

distribution of the emission is suggestive of a velocity gradient, with velocity increasing (becoming redder) from $\mathrm{E}$ to $\mathrm{W}$. The gradient is of the order of $1.4 \mathrm{~km} \mathrm{~s}^{-1} \mathrm{pc}^{-1}$. The $\mathrm{HCO}^{+}$data for the main component indicate a gradient of $\sim 0.8 \mathrm{~km} \mathrm{~s}^{-1} \mathrm{pc}^{-1}$; no clear velocity gradient is present in the CS data.

The line profiles in Fig. 1k show some low-level emission in the wings. For $\mathrm{HCO}^{+}$and $\mathrm{CS}$ we show the integrated wing emission in Fig. 24. As was found on a much smaller scale in our OVRO observations of $\mathrm{HCO}^{+}$and $\mathrm{SiO}$ (Molinari et al. 1998b), the blue and red lobes almost overlap, and we are seeing the outflow nearly pole-on.

Finally we note that, although Molinari et al. (1998a) found no radio continuum emission (at 2 and $6-\mathrm{cm}$ ) towards this object, there is an 11-cm radio continuum source (F3R 3484; integrated flux density $0.16 \mathrm{Jy}$, peak flux density $130 \mathrm{mJy}$ ) at this location (Fürst et al. 1990). Deep VLA-D array observations at 3.6-cm (Molinari et al., in preparation) reveal a double-lobed emission structure. The extent of the lobes (at a level of $\sim 5 \%$ of the peak value) is $\sim 93^{\prime \prime} \times 127^{\prime \prime}$ for the $\mathrm{W}$ lobe $\left(\int F \mathrm{~d} \nu \approx 60 \mathrm{mJy}\right)$, and $\sim 50^{\prime \prime}$ for the E lobe $\left.\int F \mathrm{~d} \nu \approx 17 \mathrm{mJy}\right)$. The $3.4-\mathrm{mm}$ continuum source (Molinari et al. 1998b) lies at the sharp E edge of the larger, westernmost radio lobe, at $\sim 21^{\prime \prime}$ to

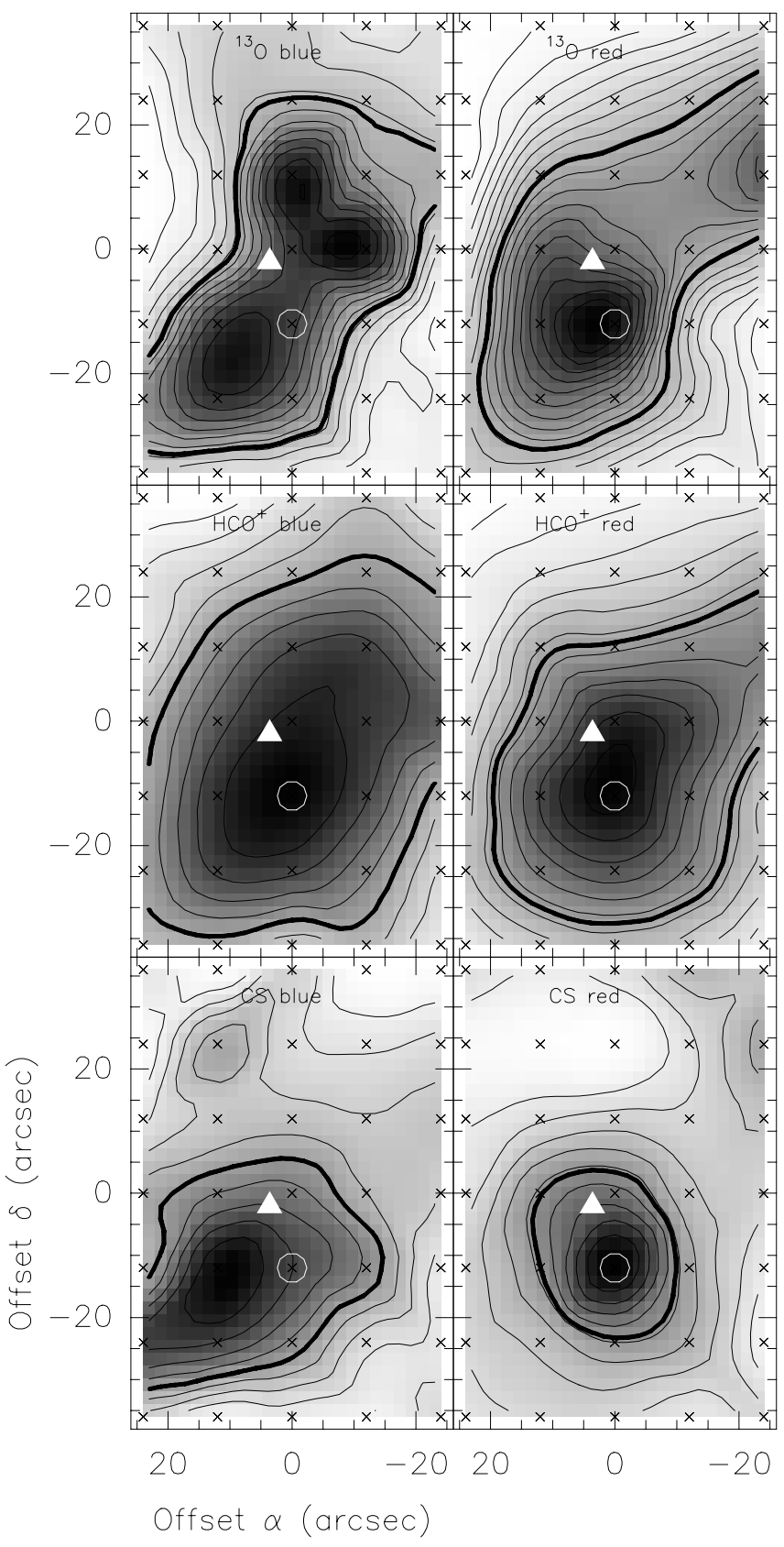

Fig. 18. Mol 117 data. Emission in the ${ }^{13} \mathrm{CO}$ (top 2 panels), $\mathrm{HCO}^{+}$(middle 2 panels), and CS (bottom 2 panels) lines, integrated over the blue and red parts of the emission (see text). Thick contours in each panel indicate the half-maximum level. The IRAS position is indicated by the triangle, the white circle is the position where longer integrations were made. Small crosses are the observed positions. Contours: ${ }^{13} \mathrm{CO}$ blue $3.5(1.5) 23 \mathrm{Kkm} \mathrm{s}^{-1}$, red 4.5(2.5)49.5; $\mathrm{HCO}^{+}$blue 0.2(0.2)2, red $0.2(0.4) 4.8$; CS blue 0.2(0.2)2, red 0.2(0.4)3.8

the NE from its peak. The general location of the radio lobes coincides with the diffuse emission seen in the $15 \mu \mathrm{m}$ ISOCAM map (see Molinari et al. 1998b), although the extent of the radio continuum emission is larger. It is unclear whether this radio emission is associated with the embedded object. 


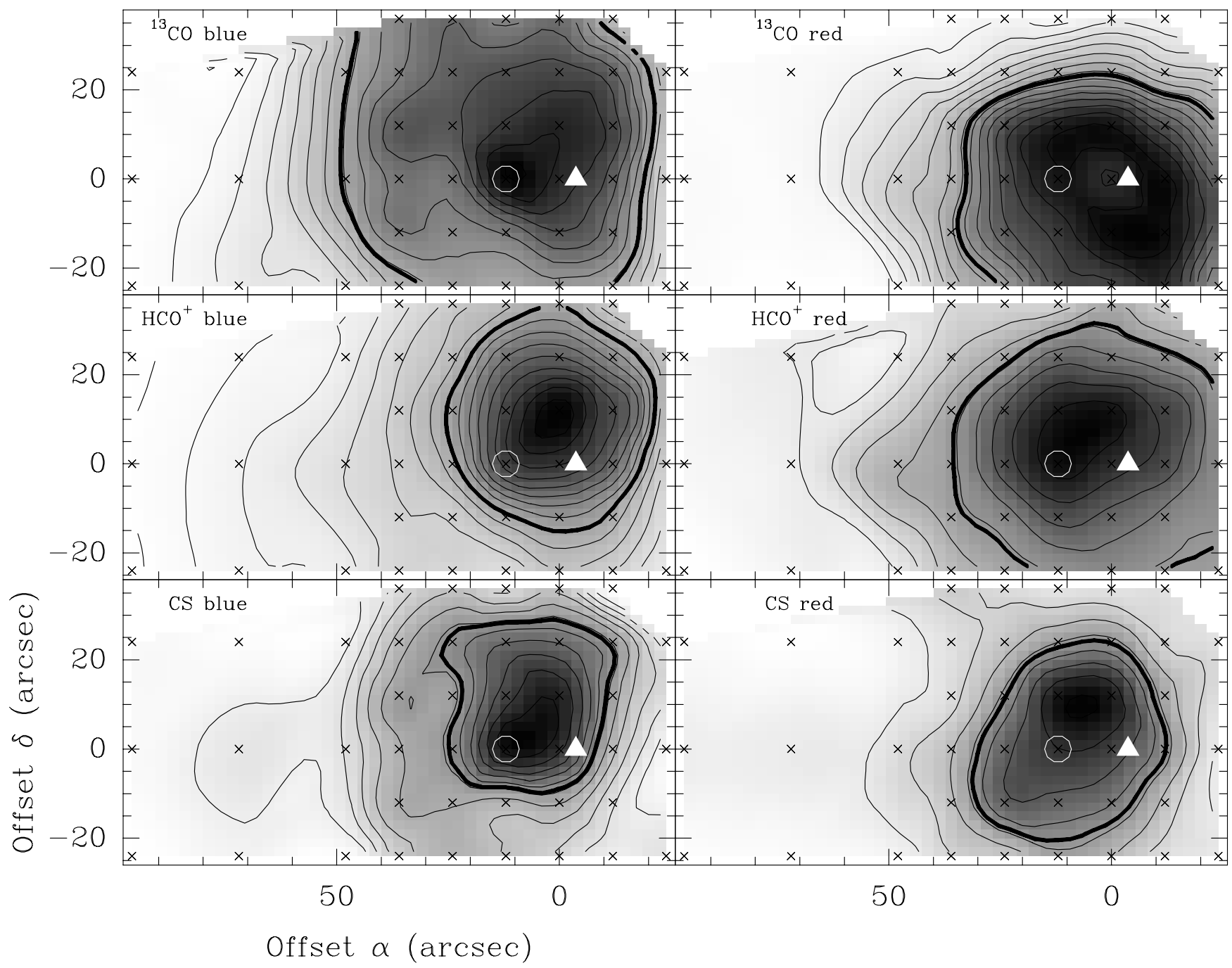

Fig. 19. Mol 118 data. Emission in the ${ }^{13} \mathrm{CO}$ (top 2 panels), $\mathrm{HCO}^{+}$(middle 2 panels), and CS (bottom 2 panels) lines, integrated over the blue and red parts of the emission (see text). Thick contours in each panel indicate the half-maximum level. The IRAS position is indicated by the triangle, the white circle is the position where longer integrations were made. Small crosses are the observed positions. Contours: ${ }^{13} \mathrm{CO}$ blue $2(3) 45 \mathrm{Kkm} \mathrm{s}^{-1}$, red $1(1) 15 ; \mathrm{HCO}^{+}$blue $0.2(0.4) 6.8$, red $0.2(0.2) 2$; CS blue 0.2(0.4)4.6, red $0.2(0.4) 3.4$

\section{Discussion and conclusions}

The most direct result of this work is that all of the observed sources are clearly associated with well-defined molecular clumps. It must be stressed that such clumps are seen in various molecular tracers and are hence real physical entities. The main goal of our observations was to obtain a picture of the molecular environment associated with Low sources and look for any evidence supporting the hypothesis that Low sources are the precursors of High sources. In this scenario, the former correspond to YSOs still accreting mass from the surrounding environment, whereas the latter are ZAMS early type stars still deeply embedded in their natal cores. The most direct way to verify this is to make high-angular resolution observations of the Low objects to study their structure and physical parameters, and indeed we are already performing this type of investigation on a limited number of objects selected on the basis of the present study. However, it is also possible to draw some tentative conclusions by comparing our sample with a sample of High sources studied by Cesaroni et al. (1999) with the same telescope and in the same lines. For this purpose, we have collected in Table 9 all the parameters derived from both studies: for each quantity we give the minimum, maximum, and mean values. We note that sources Sh-2 233 and NGC 2024 from the Cesaroni et al. (1999) sample have not been considered here, as they satisfy neither the requirements of the High-, nor of the Low sources.

In Table $9, \Theta$ is the angular diameter of the clumps after deconvolution of the beam, $D$ is the corresponding linear diameter, $T_{\mathrm{mb}}(\mathrm{K})$ and $T_{\mathrm{b}}(\mathrm{K})$ are the main beam and intrinsic (after correcting for the beam filling factor) brightness temperatures, $F W Z I$ the line full width at zero intensity, $F W H M$ the line full width at half 


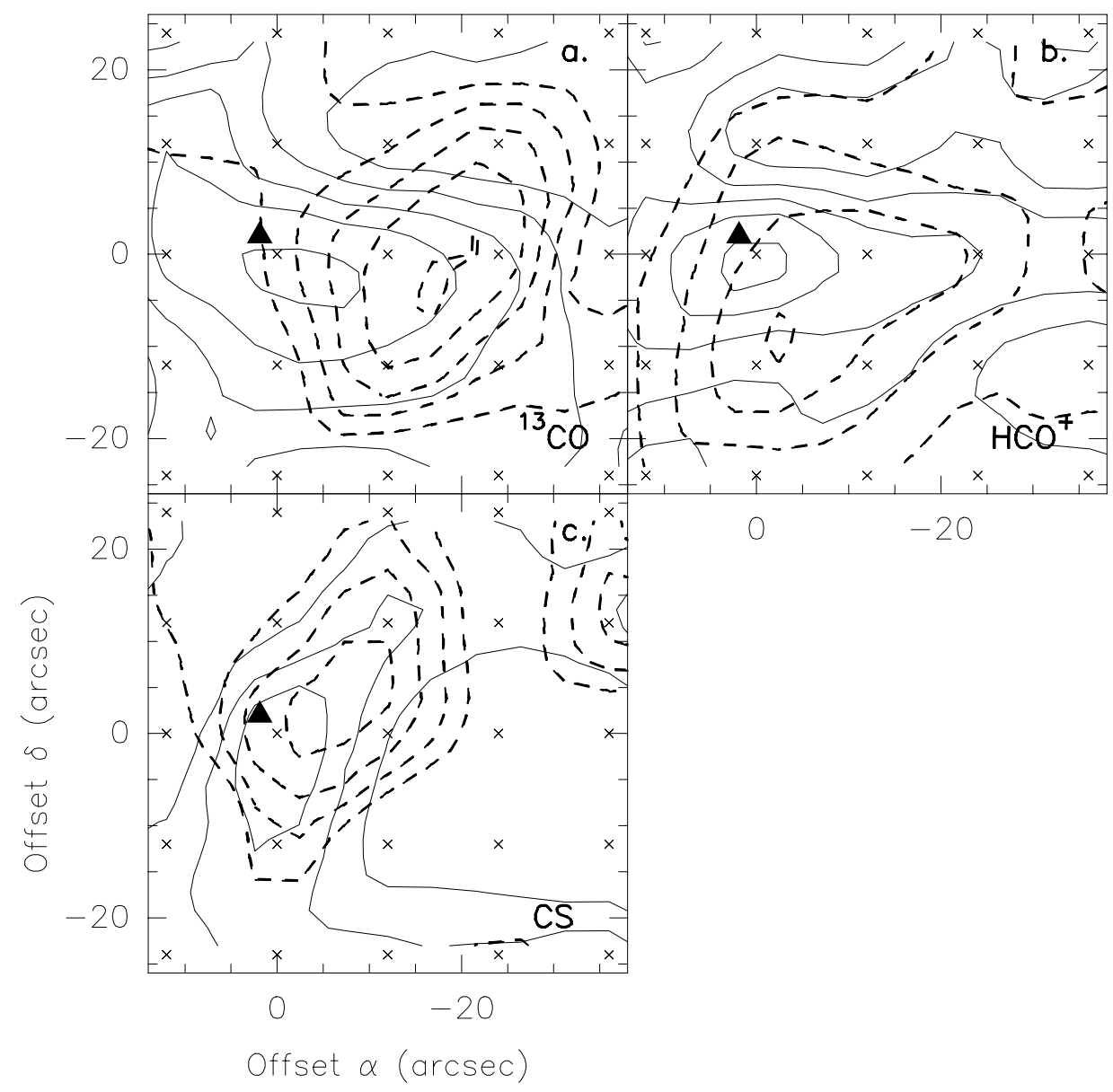

Fig. 20. Mol 136 data. Emission in the wings of the profiles of ${ }^{13} \mathrm{CO}$ a), $\mathrm{HCO}^{+} \mathbf{b}$ ), and $\mathrm{CS} \mathbf{c}$ ). Drawn and dashed contours refer to blue and red wings, respectively. Integrated velocity interval for the blue emission is -51 to -48 ; for the red emission it's -44.5 to -41 (-42 for CS). Contour values (low(step)max) are: $1(1) 5 \mathrm{Kkm} \mathrm{s}^{-1}$ a); $0.2(0.2) 1 \mathrm{Kkm} \mathrm{s}^{-1}$ (b), blue), $0.2(0.2) 0.8 \mathrm{Kkm} \mathrm{s}{ }^{-1}$ (b), red); $0.2(0.3) 1.1 \mathrm{Kkm} \mathrm{s}^{-1}$ (c), blue), and $0.2(0.2) 1 \mathrm{Kkm} \mathrm{s}^{-1}$ (c), red). The triangle indicates the position of the IRAS source

maximum, $M_{\mathrm{vir}}$ the virial mass, and $M_{\mathrm{CD}}$ the mass obtained by integrating the line emission over the line profile and over the whole emitting region. For the latter estimate we have assumed LTE at $30 \mathrm{~K}$ and abundances of $1.110^{-6}$, $10^{-9}, 10^{-8}, 4.510^{-10}$ respectively for ${ }^{13} \mathrm{CO}, \mathrm{HCO}^{+}, \mathrm{CS}$, and $\mathrm{C}^{34} \mathrm{~S}$ (Irvine et al. 1987). Note that the ${ }^{13} \mathrm{CO}$ abundance is what was used by Cesaroni et al. (1999), and is slightly smaller (by a factor of 1.8) than what we used in Sect. 5. In only four Low sources (Mol 8, 77, 98, and 160) the $\mathrm{C}^{34} \mathrm{~S}(3-2)$ emission was strong enough to be mapped; sizes, masses, and intrinsic brightness temperature derived from this molecule, reported in Table 9 are derived from these four objects only, and may not be representative.

Inspection of Table 9 reveals a few interesting differences between the two samples in spite of the similar bolometric luminosities. Relative to the clumps associated with High sources, those around the Low sources are more massive (2-4 times; except for $\left.\mathrm{C}^{34} \mathrm{~S}\right)$, larger ( $\sim 3$ times), less bright (1-3 times), have smaller line widths $(\gtrsim 1.5$ times). The Low sources also appear to be less dynamically stable than the High sources since they typically have a smaller ratio $M_{\mathrm{vir}} / M_{\mathrm{CD}}$, which is a measure of the balance between gravitation and turbulence: the smaller it is, the weaker is the support against collapse. The results are therefore consistent with Low sources representing an evolutionary phase prior to that of High sources. In fact, the larger diameters of the Low clumps suggest that they should still undergo substantial contraction, as indicated by the lower densities. Moreover, the lower brightness temperatures may be considered an indication of lower temperatures and/or optical depths, both expected in an early phase of the evolution. Finally, the broader lines in the High sample might be related to the existence of a larger number of molecular outflows and hence of YSOs already formed: such outflows could support the surrounding clump from collapse by injecting high velocity gas, thus accounting for the higher ratio $M_{\mathrm{vir}} / M_{\mathrm{CD}}$.

We note that the clump mass as reported in Table 8 increases with the $L_{\text {fir }}$ of the IRAS source (see Fig. 25a; a least-squares fit to mass versus luminosity gives a slope of $\sim 1.17 \pm 0.22$ and a corr. coeff. of $\sim 0.57)$. Then, assuming that each clump contains a single protostellar object, we can obtain an estimate of the mass of the central object from the known value of $L_{\text {fir }}$. Using the models of 


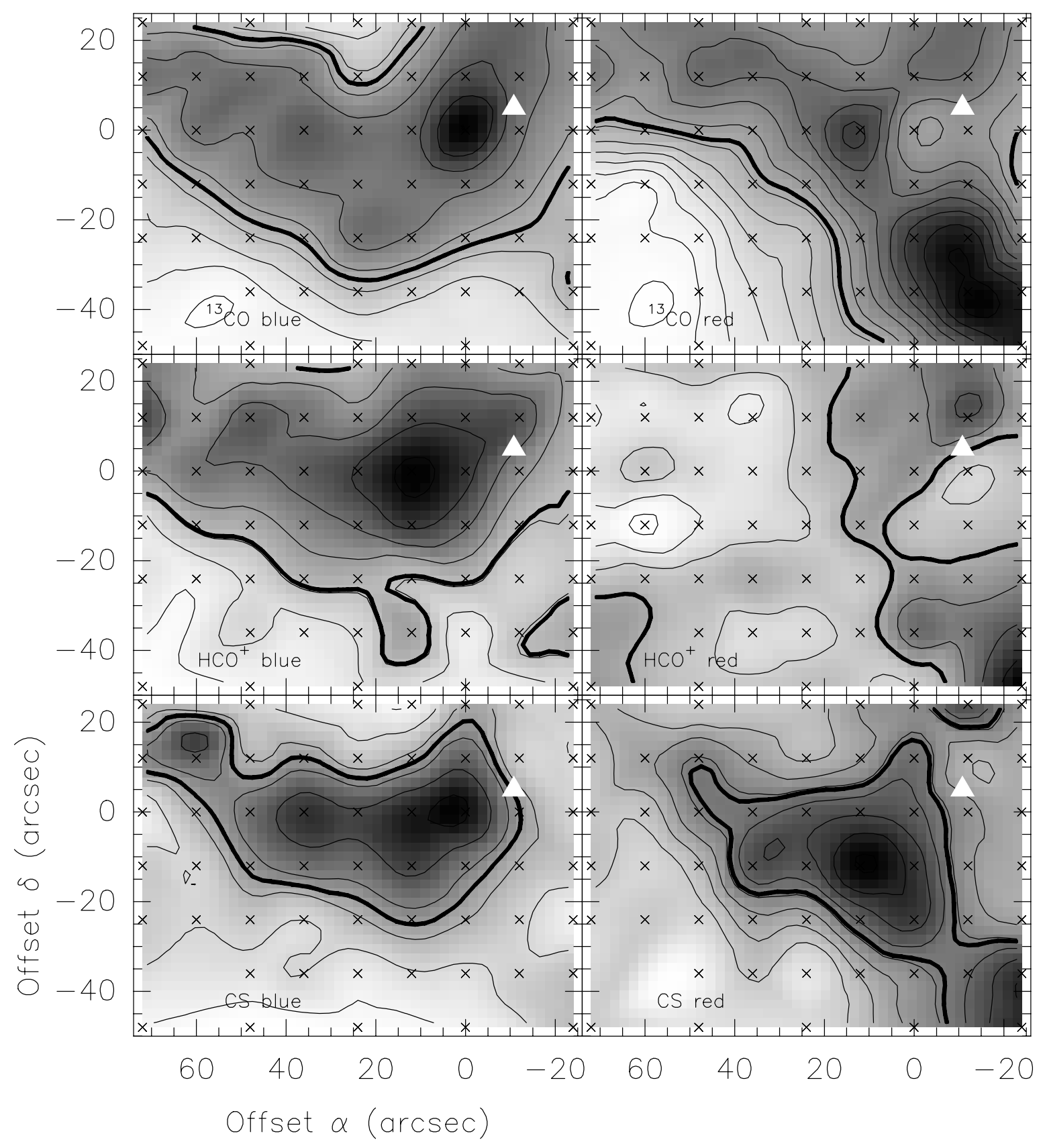

Fig. 21. Mol 155 data. Emission in the ${ }^{13} \mathrm{CO}$ (top 2 panels), $\mathrm{HCO}^{+}$(middle 2 panels), and CS (bottom 2 panels) lines, integrated over the blue and red parts of the emission (see text). Thick contours in each panel indicate the half-maximum level. The IRAS position is indicated by the triangle. Small crosses are the observed positions. Contours: ${ }^{13} \mathrm{CO}$ blue $5(5) 45 \mathrm{Kkm} \mathrm{s}{ }^{-1}$, red $3(3) 44$; $\mathrm{HCO}^{+}$blue 0.5(0.5)4, red 0.2(0.4)3; CS blue 0.2(0.8)4.4, red 0.2(0.4)3.4

Palla \& Stahler (1992) for an accretion rate of $10^{-4} M_{\odot} /$ year, we compute the mass of a protostar that produces a luminosity, $L_{\text {proto }}$, equal to the observed $L_{\text {fir }}$. Note that $L_{\text {proto }}$ includes a component from accretion and one due to gravitational contraction. For the range of luminosities considered here, the latter term dominates. The resulting protostellar masses are plotted against clump masses in Fig. 25b. The highest mass that the models of Palla \& Stahler (1992) can give is $\sim 17 M_{\odot}$, hence the two lower limits for two of the objects (Mol 3 and 8). The lowest protostellar mass is for Mol 118 and amounts to $7.4 M_{\odot}$. From Fig. $25 \mathrm{~b}$ we see that there is a weak dependence on clump mass. A fit to the data points shows that $M_{\text {proto }} \propto M_{\text {clump }}^{0.30 \pm 0.07}$. Note that the slope of this relation is similar to that found by Larson (1982) in his study of young stars and molecular clouds, who found that the maximum mass of stars is related to the mass of the cloud as $M_{\text {star }} \propto M_{\text {cloud }}^{0.43}$.

In conclusion, we believe that our results lend support to the evolutionary scenario previously proposed by us 


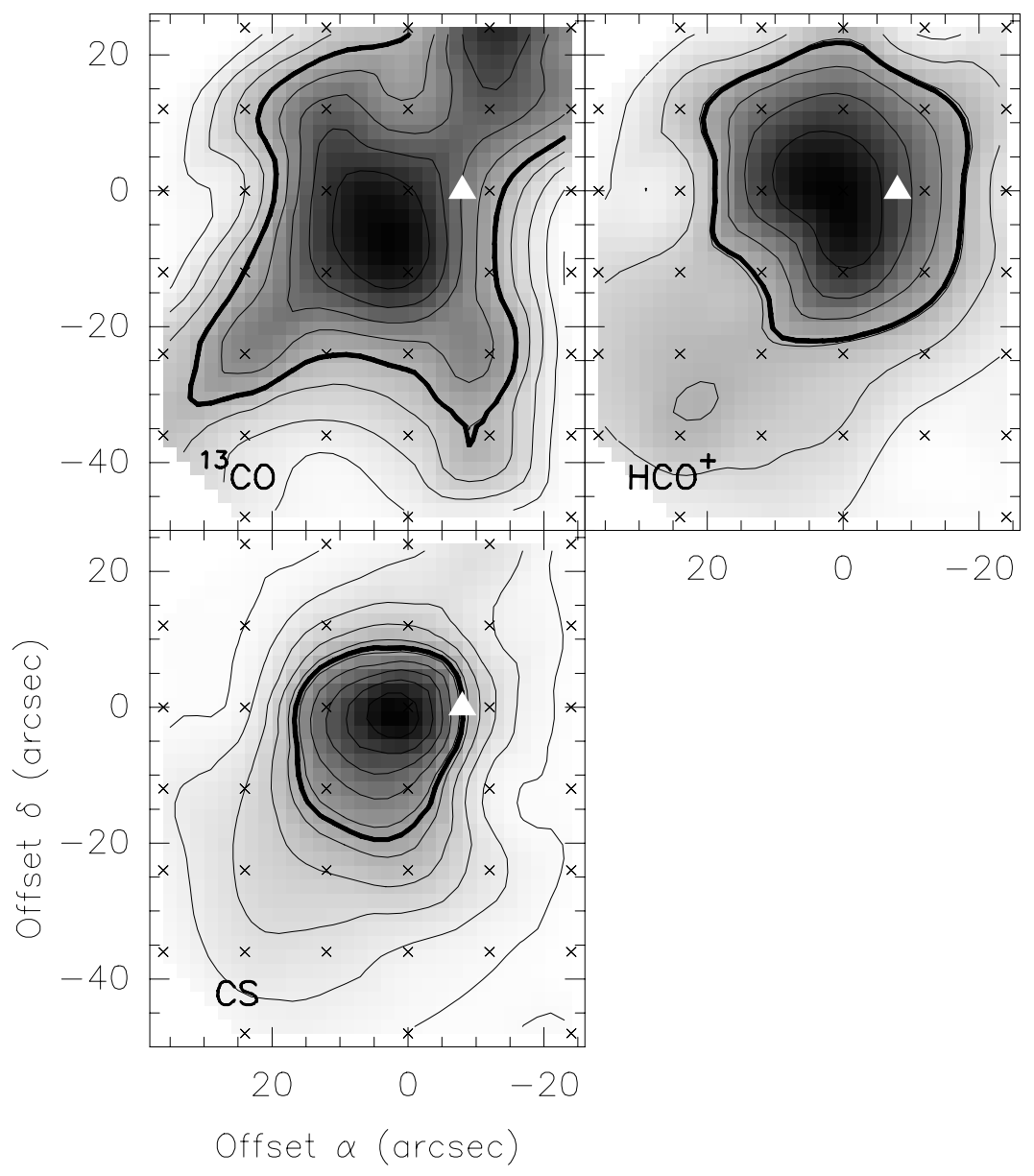

Fig. 22. Mol 160 data. Area under the main component (subtracting components using Gaussian fits), for the molecules marked in the panels. The triangle indicates the IRAS position. Contour values are $7(6) 61 \mathrm{Kkm} \mathrm{s}^{-1}$ $\left({ }^{13} \mathrm{CO}\right) ; \quad 1(2) 14 \quad \mathrm{Kkm} \mathrm{s}^{-1} \quad\left(\mathrm{HCO}^{+}\right)$; $1(2) 19 \mathrm{Kkm} \mathrm{s}^{-1}$ (CS). The thick contours mark the $F W H M$ values
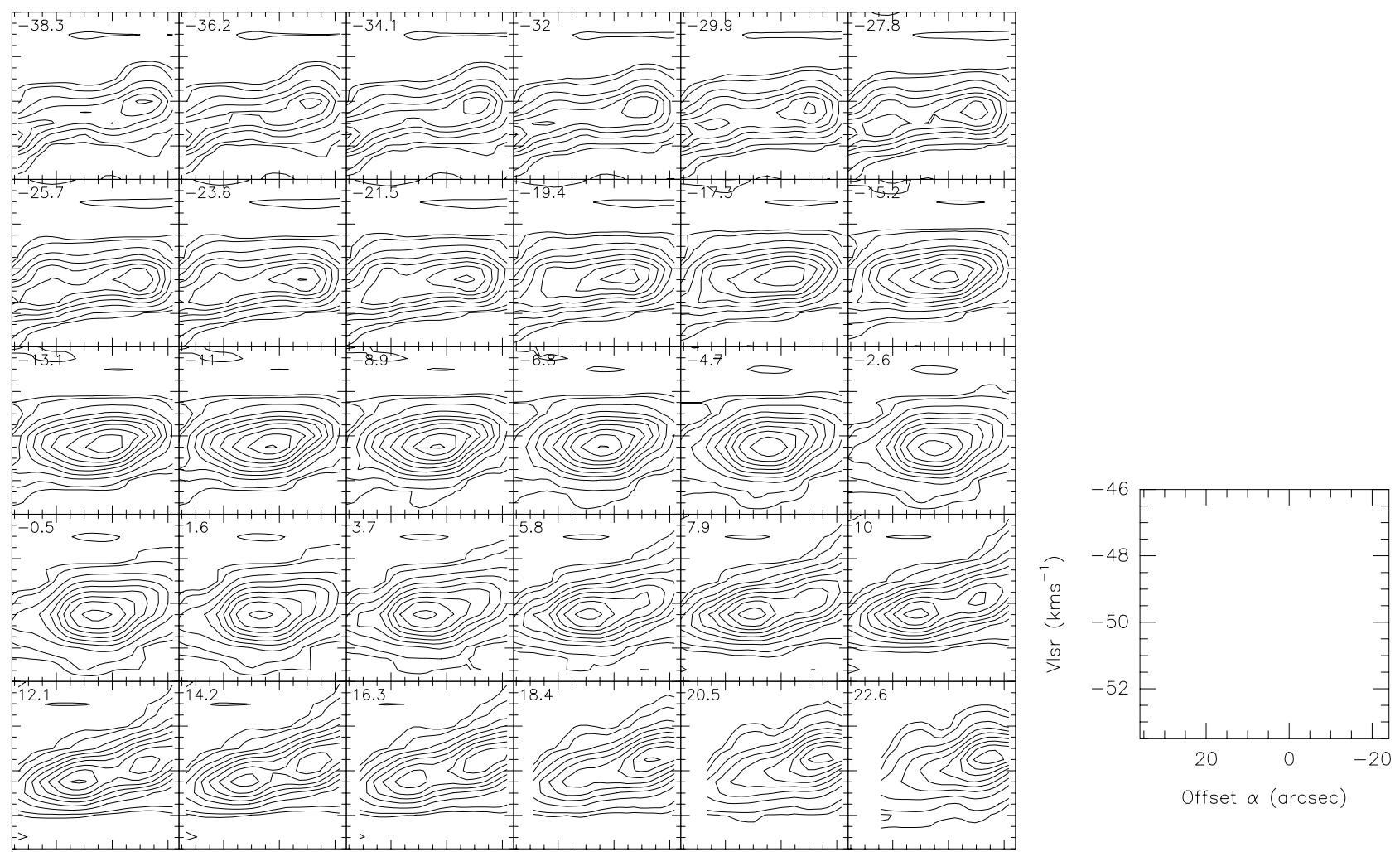

Fig. 23. Mol 160 data. Right ascension - velocity plots of the ${ }^{13} \mathrm{CO}$ line at the declination offsets marked in each panel. Contour values are 1, 2(3)27 K 


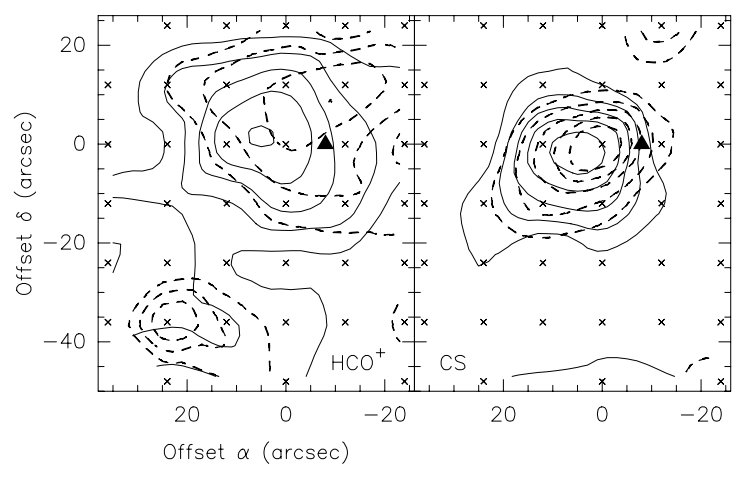

Fig. 24. Mol 160 data. Emission in the wings of the profiles of $\mathrm{HCO}^{+}$and CS. Drawn and dashed contours refer to blue and red wings, respectively. Integrated velocity interval for the blue emission is -53 to $-52\left(\mathrm{HCO}^{+}\right),-53$ to -51.5 (CS); for the red emission it's -48.5 to $-47\left(\mathrm{HCO}^{+}\right),-49$ to -47.5 (CS). Contour values (low(step)max) are: $0.2(0.2) 1 \mathrm{Kkm} \mathrm{s}^{-1}$ $\left(\mathrm{HCO}^{+}\right) ; 0.5(0.5) 3 \mathrm{Kkm} \mathrm{s}^{-1}$ (CS). The triangle indicates the position of the IRAS source
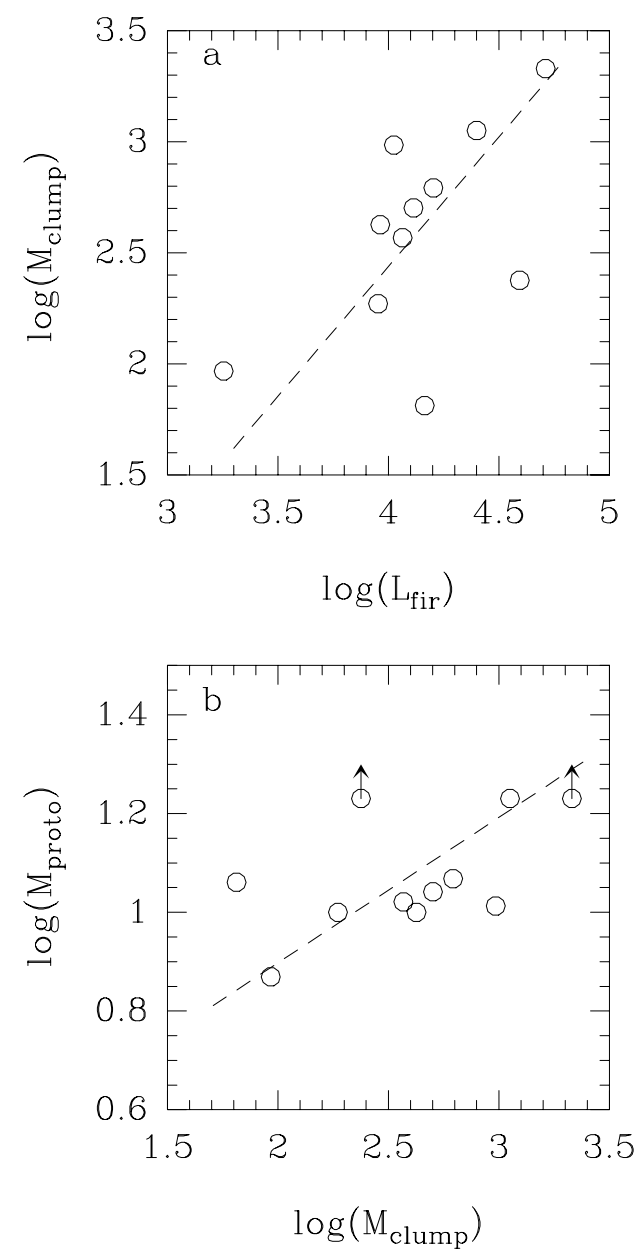

Fig. 25. a) Relation between clump mass (data from Table 8) and luminosity (data from Table 2). The least-squares (bisector) fit is shown as the dashed line, which has a slope of $1.17 \pm$ 0.22 ; b) Mass of the embedded protostellar object, as function of clump mass. The dashed line is the least-squares (bisector) fit to the data, and has a slope of $0.30 \pm 0.07$
Table 9. Comparison between properties of the Low sources observed in this study with those of the High sources observed by Cesaroni et al. (1999). The pair of numbers indicates the minimum and maximum values of the corresponding quantity, while the number below each pair is the mean value

\begin{tabular}{|c|c|c|}
\hline & Low & High \\
\hline$d(\mathrm{kpc})$ & $\begin{array}{c}1.6-10.8 \\
5.9\end{array}$ & $\begin{array}{c}0.7-3.5 \\
1.5\end{array}$ \\
\hline $\log \left[L\left(L_{\odot}\right)\right]$ & $3.3-4.7$ & $2.6-4.5$ \\
\hline \multicolumn{3}{|c|}{$\mathrm{CS}(3-2)$} \\
\hline$\Theta\left({ }^{\prime \prime}\right)$ & $\begin{array}{c}13-51 \\
28\end{array}$ & $\begin{array}{c}18-38 \\
29\end{array}$ \\
\hline$D(\mathrm{pc})$ & $\begin{array}{c}0.32-1.46 \\
0.75\end{array}$ & $\begin{array}{c}0.13-0.31 \\
0.22\end{array}$ \\
\hline$T_{\mathrm{mb}}(\mathrm{K})$ & $\begin{array}{c}2.1-6.0 \\
3.4\end{array}$ & $\begin{array}{c}3.2-12.9 \\
9.4\end{array}$ \\
\hline$T_{\mathrm{b}}(\mathrm{K})$ & $\begin{array}{c}2.5-8.2 \\
5.4\end{array}$ & $\begin{array}{c}3.9-18.0 \\
13.5\end{array}$ \\
\hline$F W Z I\left(\mathrm{~km} \mathrm{~s}^{-1}\right)$ & $\begin{array}{c}3.5-11.6 \\
5.9\end{array}$ & $\begin{array}{c}18-52 \\
33\end{array}$ \\
\hline$F W H M\left(\mathrm{~km} \mathrm{~s}^{-1}\right)$ & $\begin{array}{c}1.8-6.1 \\
2.9\end{array}$ & $\begin{array}{c}2.4-4.4 \\
3.4\end{array}$ \\
\hline$M_{\mathrm{vir}}\left(M_{\odot}\right)$ & $\begin{array}{c}121-1797 \\
629\end{array}$ & $\begin{array}{c}95-479 \\
276\end{array}$ \\
\hline$M_{\mathrm{CD}}\left(M_{\odot}\right)$ & $\begin{array}{c}7.6-165 \\
92.3\end{array}$ & $\begin{array}{c}3.1-55 \\
31.5\end{array}$ \\
\hline$M_{\mathrm{CD}}^{*}\left(M_{\odot}\right)$ & $\begin{array}{c}16-1167 \\
405\end{array}$ & $\begin{array}{c}12-177 \\
81\end{array}$ \\
\hline$M_{\mathrm{vir}} / M_{\mathrm{CD}}$ & $\begin{array}{c}2.6-24.6 \\
9.0\end{array}$ & $\begin{array}{c}4.8-30.6 \\
13.6\end{array}$ \\
\hline$M_{\mathrm{vir}} / M_{\mathrm{CD}}^{*}$ & $\begin{array}{c}0.4-7.6 \\
2.5\end{array}$ & $\begin{array}{c}2.3-8.0 \\
4.8\end{array}$ \\
\hline & $(3-2)$ & \\
\hline$\Theta\left({ }^{\prime \prime}\right)^{*}$ & $\begin{array}{c}8-17 \\
13\end{array}$ & $\begin{array}{c}14-34 \\
23\end{array}$ \\
\hline$D(\mathrm{pc})^{\star}$ & $\begin{array}{c}0.22-0.9 \\
0.44\end{array}$ & $\begin{array}{c}0.12-0.36 \\
0.21\end{array}$ \\
\hline$T_{\mathrm{mb}}(\mathrm{K})$ & $\begin{array}{c}0.1-1.2 \\
0.6\end{array}$ & $\begin{array}{c}0.5-2.5 \\
1.4\end{array}$ \\
\hline$T_{\mathrm{b}}(\mathrm{K})^{\boldsymbol{中}}$ & $\begin{array}{c}1.3-2.4 \\
1.8\end{array}$ & $\begin{array}{c}1.2-3.2 \\
2.4\end{array}$ \\
\hline$F W H M\left(\mathrm{~km} \mathrm{~s}^{-1}\right)$ & $\begin{array}{c}1.0-4.1 \\
2.0\end{array}$ & $\begin{array}{c}2.2-4.2 \\
3.1\end{array}$ \\
\hline$M_{\text {vir }}\left(M_{\odot}\right)^{\boldsymbol{q}}$ & $\begin{array}{c}59-160 \\
202\end{array}$ & $\begin{array}{c}87-370 \\
227\end{array}$ \\
\hline$M_{\mathrm{CD}}\left(M_{\odot}\right)^{\boldsymbol{\mu}}$ & $\begin{array}{c}86-229 \\
143\end{array}$ & $\begin{array}{c}41-354 \\
170\end{array}$ \\
\hline$M_{\mathrm{vir}} / M_{\mathrm{CD}}^{\mathrm{h}}$ & $\begin{array}{c}0.7-3.5 \\
1.5\end{array}$ & $\begin{array}{c}0.38-5.0 \\
2.1\end{array}$ \\
\hline
\end{tabular}

* mass obtained after correcting for line optical depth: $\tau_{\mathrm{CS}} /[1-$ $\left.\exp \left(-\tau_{\mathrm{CS}}\right)\right]$, with $\tau_{\mathrm{CS}}$ from $\mathrm{T}(\mathrm{CS}) / \mathrm{T}\left(\mathrm{C}^{34} \mathrm{~S}\right)$ and $\tau_{\mathrm{C}^{34} \mathrm{~S}}=\tau_{\mathrm{CS}} / f$, $f=[\mathrm{CS}] /\left[\mathrm{C}^{34} \mathrm{~S}\right]=22$.

* Values for Low based on Mol 8, 77, 98, 160 only. 
Table 9. continued

\begin{tabular}{|c|c|c|}
\hline \multicolumn{3}{|c|}{${ }^{13} \mathrm{CO}(2-1)$} \\
\hline \multirow[t]{2}{*}{$\Theta\left({ }^{\prime \prime}\right)$} & $25-93$ & $30-95$ \\
\hline & 55 & 72 \\
\hline \multirow[t]{2}{*}{$D(\mathrm{pc})$} & $0.51-2.21$ & $0.17-0.83$ \\
\hline & 1.5 & 0.47 \\
\hline \multirow{2}{*}{$T_{\mathrm{mb}}(\mathrm{K})$} & $9.1-28.0$ & $14.8-38.2$ \\
\hline & 19.0 & 26.6 \\
\hline \multirow[t]{2}{*}{$F W Z I\left(\mathrm{kms}^{-1}\right)$} & $4.8-12.9$ & $7.6-14.4$ \\
\hline & 8.3 & 11.2 \\
\hline \multirow[t]{2}{*}{$F W H M\left(\mathrm{~km} \mathrm{~s}^{-1}\right)$} & $1.5-5.5$ & $2.8-4.4$ \\
\hline & 3.3 & 3.5 \\
\hline \multirow[t]{2}{*}{$M_{\text {vir }}\left(M_{\odot}\right)$} & $348-3557$ & $160-1591$ \\
\hline & 1702 & 628 \\
\hline \multicolumn{3}{|c|}{$\mathrm{HCO}^{+}(1-0)$} \\
\hline \multirow[t]{2}{*}{$\Theta\left({ }^{\prime \prime}\right)$} & $23-81$ & $26-41$ \\
\hline & 43 & 35 \\
\hline \multirow[t]{2}{*}{$D(\mathrm{pc})$} & $0.34-2.03$ & $0.12-0.63$ \\
\hline & 1.17 & 0.30 \\
\hline \multirow[t]{2}{*}{$T_{\mathrm{mb}}(\mathrm{K})$} & $1.3-5.8$ & $1.5-13.2$ \\
\hline & 2.4 & 7.4 \\
\hline \multirow[t]{2}{*}{$F W Z I\left(\mathrm{~km} \mathrm{~s}^{-1}\right)$} & $5.5-16.3$ & $34.0-108.3$ \\
\hline & 9.1 & 57.4 \\
\hline \multirow[t]{2}{*}{$F W H M\left(\mathrm{~km} \mathrm{~s}^{-1}\right)$} & $1.3-5.7$ & $1.3-4.5$ \\
\hline & 2.5 & 3.2 \\
\hline \multirow[t]{2}{*}{$M_{\text {vir }}\left(M_{\odot}\right)$} & $212-1979$ & $22-1335$ \\
\hline & 757 & 440 \\
\hline \multirow[t]{2}{*}{$M_{\mathrm{CD}}\left(M_{\odot}\right)$} & $97-1838$ & $32-360$ \\
\hline & 922 & 223 \\
\hline \multirow[t]{2}{*}{$M_{\mathrm{vir}} / M_{\mathrm{CD}}$} & $0.2-2.7$ & $0.66-5.8$ \\
\hline & 1.1 & 2.3 \\
\hline
\end{tabular}

(Palla et al. 1991; Molinari et al. 1996; Molinari et al. 1998a), according to which the majority of Low sources will eventually evolve into High sources.

Acknowledgements. We thank Jan Wouterloot for the KOSMA observations. The KOSMA radio telescope at GornergratSüd Observatory is operated by the University of Köln, and supported by the Deutsche Forschungsgemeinschaft through grant SFB-301, as well as by special funding from the Land Nordrhein-Westfalen. The Observatory is administered by the Internationale Stiftung Hochalpine Forschungsstationen Jungfraujoch und Gornergrat, Bern, Switzerland.

\section{References}

André, P. 1994, in The Cold Universe, Proc. XIIIth Moriond Astrophysics Meetings, ed. T. Montmerle, C. J. Lada, I. F. Mirabel, \& J. Trân Thanh Vân, 179

Bergin, E. A., Goldsmith, P. F., Snell, R. L., \& Ungerechts, H. 1994, ApJ, 431, 674

Brand, J., \& Blitz, L. 1993, A\&A, 275, 67

Brand, J., \& Wouterloot, J. G. A. 1998, A\&A, 337, 539

Campbell, B., Persson, S. E., \& Matthews, K. 1989, AJ, 98, 643
Cesaroni, R., Felli, M., Testi, L., Olmi, L., \& Walmsley, C. M. 1997, A\&A, 325, 725

Cesaroni, R., Felli, M., \& Walmsley, C. M. 1999, A\&AS, 136, 333

Codella, C., Felli, M., \& Natale, V. 1996, A\&A, 311, 971

Codella, C., Testi, L., \& Cesaroni, R. 1997, A\&A, 325, 282

Felli, M., Palagi, F., \& Tofani, G. 1992, A\&A, 255, 293

Fürst, E., Reich, W., Reich, P., \& Reif, K. 1990, A\&AS, 85, 691

Harju, J., Lehtinen, K., Booth, R. S., \& Zinchenko, I. 1998, A\&AS, 132, 211

Irvine, W. M., Goldsmith, P. F., \& Hjalmarson, A. 1987, Chemical abundances in molecular clouds, in Interstellar Processes, ed. D. J. Hollenbach, \& H. A. Thronson (Reidel, Dordrecht), 561

Ishii, M., Nagata, T., Sato, S., Watanabe, M., \& Yao, Y. 1998, AJ, 116, 868

Jenness, T., Scott, P. F., \& Padman, R. 1995, MNRAS, 276, 1024

Kuiper, T. B. H., Rodriguez-Kuiper, E. N., \& Dickinson, D. F. 1984, ApJ, 276, 211

Larson, R. B. 1982, MNRAS, 200, 159

MacLeod, G., van der Walt, D. J., North, A., Gaylard, M. J., \& Moriarty-Schieven, G. H. 1998, AJ, 116, 2936

Molinari, S., Brand, J., Cesaroni, R., \& Palla, F. 1996, A\&A, 308,573

Molinari, S., Brand, J., Cesaroni, R., Palla, F., \& Palumbo, G. G. C. $1998 \mathrm{a}, \mathrm{A} \& \mathrm{~A}, 336,339$

Molinari, S., Testi, L., Brand, J., Cesaroni, R., \& Palla, F. 1998b, ApJL, 505, L39

Molinari, S., Brand, J., Cesaroni, R., \& Palla, F. 2000, A\&A, 355,617

Myers, P. J. C., Evans, N. J., \& Ohashi, N. 2000, Observations of infall in star-forming regions, in Protostars and Planets IV, ed. V. Mannings, A. P. Boss, \& S. S. Russell (Tucson: Univ. of Arizona Press), 217

Neckel, T., \& Staude, H. J. 1984, A\&A, 131, 200

Neckel, T., Vehrenberg, H. 1985, 1987, 1990, Atlas of Galactic Nebulae, vols. I - III (Treugesell Verlag, Düsseldorf)

Palla, F., Brand, J., Cesaroni, R., \& Comoretto, G. 1991, A\&A, 246, 249

Palla, F., Cesaroni, R., Brand, J., Comoretto, G., \& Felli, M. 1993, A\&A, 280, 599

Palla, F., \& Stahler, S. W. 1992, ApJ, 392, 667

Richards, P. J., Little, L. T., Toriseva, M., \& Heaton, B. D. 1987, MNRAS, 228, 43

Rohlfs, K., \& Wilson, T. L. 1996, Tools of Radio Astronomy (Springer-Verlag, Berlin)

Shepherd, D. S., \& Churchwell, E. 1996, ApJ, 457, 267

Slysh, V. I., Dzura, A. M., Val'tts, I. E., \& Gerard, E. 1997, A\&AS, 124, 85

Valdettaro, R., Palla, F., Brand, J., et al. 2001, A\&A, 368, 845

White, G. J., \& Gee, G. 1986, A\&A, 156, 301

Wilson, T. L., \& Rood, R. T. 1994, ARA\&A, 32, 191

Wood, D. O. S., \& Churchwell, E. 1989, ApJ, 340, 265

Wouterloot, J. G. A., \& Brand, J. 1989, A\&AS, 80, 149

Wouterloot, J. G. A., Fiegle, K., Brand, J., \& Winnewisser, G. 1995, A\&A, 301, 236 